\title{
Secondary power operations and the Brown-Peterson spectrum at the prime 2
}

\author{
Tyler Lawson*
}

May 4, 2018

\begin{abstract}
The dual Steenrod algebra has a canonical subalgebra isomorphic to the homology of the Brown-Peterson spectrum. We will construct a secondary operation in mod-2 homology and show that this canonical subalgebra is not closed under it. This allows us to conclude that the 2-primary Brown-Peterson spectrum does not admit the structure of an $E_{n}$-algebra for any $n \geq 12$, answering a question of May in the negative.
\end{abstract}

\section{Introduction}

The following appeared as Problem 1 in J.P. May's "Problems in infinite loop space theory" [May75].

Problem 1.0.1. For any primep, does the p-local Brown-Peterson spectrum BP of [BP66] admit the structure of an $E_{\infty}$-algebra?

Our goal in this paper is to address this question when $p=2$. We will construct a secondary operation in the homology of $E_{\infty}$-algebras at the prime 2 and show, with an analysis that begins with the calculations of Johnson-Noel [JN10], that the homology $H_{*} B P$ cannot admit such a secondary operation. Thus, Problem 1.0.1 has a negative answer at the prime 2 .

\subsection{Background}

Coherently commutative multiplication structures have a long history in homotopy theory, originating in the study of the cup product. The cup product in the cohomology of a space $X$ comes from the structure of a differential graded algebra on the cochains $C^{*}(X)$, and while there are many variants on this algebra structure that all give rise to the cup product there is no natural cochain-level cup product that is graded-commutative. Instead, the cup product $\alpha \smile \beta$ and its reverse $\pm \beta \smile \alpha$ are chain homotopic by a natural operation $\alpha \smile_{1} \beta$, called the cup-1 product. The cup-1 product

${ }^{*}$ The author was partially supported by NSF grant 1610408 . 
is not graded-commutative either, but differs from its reverse by an operation called the cup-2 product, and these constructions extend out both to arbitrarily high "coherences" (giving cup- $i$ products for all $i$ ) and to operations accepting arbitrarily many inputs (giving a more complicated set of operations of several variables discussed in [MS04]). The result is called an $E_{\infty}$-algebra structure and Steenrod's reduced power operations in the cohomology of spaces are built from it [Ste62].

Since then, these coherently commutative multiplications have been recognized in many other areas: iterated loop spaces, monoidal structures on categories, structures in mathematical physics related to string theory, and multiplications in cohomology theories. By contrast with algebra, where commutativity is simply a property of a ring, coherent multiplications come in a hierarchy: there are $E_{1}$-algebra structures that correspond to associative products and there are $E_{\infty}$-algebra structures that correspond to commutative products, but there are also $E_{n}$-algebra structures for $1<n<\infty$ that interpolate between these concepts.

When we switch from ordinary cohomology to generalized cohomology theories, chain complexes become replaced with spectra. A ring spectrum $R$ is a representing object for a cohomology theory $R^{*}(-)$ so that cohomology with coefficients in $R$ naturally takes values in rings. This was refined to the concept of an $E_{n}$-algebra structure on the spectrum $R$ in [BMMS86, I.4], and these more refined algebras have come to occupy a central role because $E_{n}$-algebra structures produce concrete tools that are not available to an ordinary ring spectrum [Man12, Lur17].

- An $E_{1}$-algebra $R$ can be given categories of left $R$-modules and right $R$-modules, whose homotopy categories are triangulated categories. These enjoy several forms of compatibility as $R$ varies, extend to categories of bimodules, and have relative smash products $\wedge_{R}$ with properties much like the tensor product.

- The category of left modules over an $E_{2}$-algebra $R$ is canonically equivalent to the category of right modules, and the smash product $\wedge_{R}$ makes the category of left $R$-modules into a monoidal category. The homotopy category of left $R$-modules has the structure of a (neither symmetric nor braided) tensor triangulated category.

- The homotopy category of left modules over an $E_{3}$-algebra has the structure of a braided monoidal category.

- The homotopy category of left modules over an $E_{4}$-algebra has the structure of a symmetric monoidal category.

- The category of modules over an $E_{\infty}$-algebra $R$ has homotopy-theoretic versions of symmetric power operations, making it possible to discuss a relative version of the above: we can define $E_{n} R$-algebras which satisfy all of the above properties.

- An $E_{\infty}$-algebra $R$ has, for any principal $\Sigma_{n}$-bundle $P \rightarrow B$, natural geometric power operations $R^{0}(X) \rightarrow R^{0}\left(P \times_{\Sigma_{n}} X^{n}\right)$ and $R^{0}(X) \rightarrow R^{0}(B \times X)$ in $R$ cohomology that enhance the multiplicative structure. When $P=\Sigma_{n}$ these recover the operation that sends a class to its $n$th power. 
Many examples of $E_{\infty}$-algebras exist. Commutative rings $A$ produce $E_{\infty}$-algebras $H A$ via the Eilenberg-Mac Lane construction; the spectra $K O$ and $K U$, representing real and complex $K$-theory, have $E_{\infty}$-algebra structures whose origin is the tensor product of vector bundles; bordism spectra like $M O, M S O, M U$, and the like have $E_{\infty}$-algebra structures whose origin is the product structure on manifolds; if $Y$ is an infinite loop space, then there is a spherical group algebra $\mathbb{S}[Y]=\Sigma_{+}^{\infty} Y$ with an $E_{\infty}$-algebra structure; and if $R$ is an $E_{\infty}$-algebra and $X$ is a space, there is a spectrum $R^{X}$ (playing the role of "cochains on $X$ with coefficients in $R$ ") with an $E_{\infty}$-algebra structure that combines the multiplication on $R$ with the diagonal map $X \rightarrow X \times X$.

Problem 1.0.1 dates back to the first systematic studies of $E_{\infty}$-algebras. Understanding why this result is so desirable requires knowing a little about what the BrownPeterson spectrum is and how important it is in stable homotopy theory.

The complex bordism spectrum $M U$ has an $E_{\infty}$-algebra structure and it is central to Quillen's relation between stable homotopy theory and formal group laws [Qui69], which initiated the subject of chromatic homotopy theory. However, while almost the entirety of chromatic theory is possible to phrase in terms of $M U$, the $p$ localization $M U_{(p)}$ decomposes into summands equivalent to this irreducible BrownPeterson spectrum BP. The Brown-Peterson spectrum has simpler cohomology and homotopy groups than $M U$ and has canonical descriptions that are internal to the stable homotopy category [Pri80]. The Brown-Peterson spectrum also exhibits the close connection between $p$-local stable homotopy theory and the theory of formal group laws, but with the added benefit that nearly every deep structural property of chromatic homotopy theory or formal group law theory is made more concise and more conceptually accessible through the eyes of BP-theory (see, for example, [Rav86] for extensive applications).

The existence of an $E_{\infty}$-algebra structure on $B P$ would be useful in several ways.

- The Adams-Novikov spectral sequence is a method for calculating the set of homotopy classes of maps between two spectra $X$ and $Y$ and can be derived from either their $M U$-homology or $B P$-homology. The computational tools using $M U$-theory (such as the cobar complex) are well behaved with respect to the geometric power operations discussed earlier, which appear in places such as the construction of manifolds of Kervaire invariant one [Bru01]. If $B P$ had an $E_{\infty^{-}}$ algebra structure then computations of these geometric power operations using $M U$-theory could instead be related to simpler computations in BP-theory.

- Such a structure would allow more concise constructions of many important objects in chromatic theory, such as the Morava $K$-theories $K(n)$ and the truncated Brown-Peterson spectra $\mathrm{BP}\langle n\rangle$, as $B P$-algebras rather than as $M U$-algebras.

- These algebra structures would mean that several computations with these ring spectra could be governed by the computations for BP-theory, such as computations of topological Hochschild homology and topological cyclic homology that are important to current work in algebraic $K$-theory [AR02]. These can also be extended by relative computations in $B P$-modules, which are much simpler than the relative calculations in $M U$-modules. 
- Perhaps most importantly, the Brown-Peterson spectrum is one of the most prominent examples of an important homology theory where our knowledge of geometric interpretations (e.g. via Baas-Sullivan theory [Baa73]) lags far behind our algebraic knowledge. ${ }^{1}$ Many of the prominent examples of $E_{\infty}$ algebras, such as $K$-theory and bordism theory, originate in cohomology theories with geometric cycles or cocycles that have a product. The existence of an $E_{\infty}$-algebra structure on $B P$ would be a good indicator that a strong geometric interpretation existed.

This problem has generated a great deal of interesting research. The existence of multiplication structures in the homotopy category has a long history (for example, see the introduction of [Str99]). Several forms of obstruction theory have been developed which showed that many spectra constructed by Baas-Sullivan theory admit $E_{1}$ algebra structures [Rob89, Laz01, BJ02, Ang08]. More sophisticated obstruction theory has appeared for $E_{\infty}$-algebras [Rob03, GH04], and Richter obtained lower bounds on the amount of commutativity present in $B P$ based on Robinson's obstruction theory [Ric06]. Techniques such as localization and idempotent splitting were developed in [May01] to handle additive and multiplicative versions of the construction of $B P$. More recently Basterra-Mandell showed that $B P$ is a split summand of $M U_{(p)}$ as an $E_{4}$-algebra [BM13], and so the homotopy category of $B P$-modules has a symmetric monoidal structure; Chadwick-Mandell used idempotent splittings to show that this could be done with the Quillen idempotent as $E_{2}$-algebras [CM15]. Both Hu-KrizMay [HKM01] and Baker [Bak14] gave iterative constructions by methods that kill torsion, producing two different types of closest possible torsion-free $E_{\infty}$-algebra to $B P$. An unpublished paper of Kriz attempted to prove that $B P$ admits an $E_{\infty}$-algebra structure, and Basterra developed the theory of topological André-Quillen (TAQ) cohomology based on his ideas-this theory allows the construction of $E_{\infty}$-algebras by systematically lifting the $k$-invariants in the Postnikov tower from ordinary cohomology to TAQ-cohomology [Kri95, Bas99]. Kriz's original program foundered on a technical detail, but TAQ has been central in a great deal of research since. ${ }^{2}$

However, the hope that Problem 1.0.1 has a positive solution perhaps originated in a time of much greater optimism, and the intervening years have shown that the additive and multiplicative structure of a spectrum are difficult to untangle from each other. Indeed, there is something closer to a reciprocity relationship, where requirements of the additive structure are rewarded with constraints on the multiplicative and vice versa. In line with this, there have been several more recent calculations showing that desirable properties of a multiplication on $B P$ cannot be realized. $\mathrm{Hu}-$ Kriz-May showed that there cannot be a map of $E_{\infty}$-algebras $B P \rightarrow M U_{(p)}$ because it conflicts with calculation of Dyer-Lashof operations in their homology, despite the presence of the Quillen idempotent which describes such a splitting additively and algebraically [HKM01]. In the reverse direction, Johnson-Noel showed with hard calculations that the particular map of ring spectra $M U_{(p)} \rightarrow B P$ employed to great effect

\footnotetext{
${ }^{1}$ From [May75]: "The point here is that the notion of an $E_{\infty}$ ring spectrum seems not to be a purely homotopical one; good concrete geometric models are required, and no such model is known for $B P$."

${ }^{2}$ The problem, insofar as the author understands, was establish certain elements in the Miller spectral sequence computing TAQ-cohomology needed to be shown to be permanent cycles, but the operations used to establish this were insufficiently compatible with the differentials.
} 
in chromatic theory cannot be a map of $E_{\infty}$-algebras for $p \leq 13$ [JN10], based on a power operation criterion due to McClure [BMMS86, VIII.7.7, 7.8].

The Hu-Kriz-May result seems more decisive mainly because it uses structure that is forced. The mod- $p$ homology groups $H_{*} B P$ are identified as a canonical subalgebra of the dual Steenrod algebra $H_{*} \mathrm{HF}_{p}$, and this means that the ring structure on $H_{*} B P$ and operations coming from any $E_{n}$-algebra structure (including the DyerLashof operations mentioned above) are completely determined by those in the dual Steenrod algebra. It is straightforward to show that $H_{*} B P$ is closed in $H_{*} \mathrm{HF}_{p}$ under the Dyer-Lashof operations, and so we cannot exclude the possibility that $B P$ is an $E_{\infty}$-algebra using a relation between these primary operations. This paper shows that, at the prime 2, there does exist a contradiction for a more subtle reason: while $H_{*} B P$ is closed under primary operations, it is not closed under secondary operations. This parallels Adams' solution of the Hopf invariant one problem using secondary cohomology operations [Ada66]. The proof will critically rely on Johnson-Noel's calculation of power operations in complex bordism.

Theorem 1.1.1 (5.4.2, 5.4.5). There exists a natural secondary operation in the mod2 homology of $E_{12}$-algebras with the following properties. For $R$ an $E_{12}$-algebra, the secondary operation is defined on the subset of $\mathrm{H}_{2} R$ satisfying certain identities between Dyer-Lashof operations in $H_{k} R$ for $5 \leq k \leq 13$, and the secondary operation takes values in a quotient of $H_{31} R$. This operation is preserved by maps $R \rightarrow R^{\prime}$ of $E_{12}$-algebras.

In the dual Steenrod algebra $H_{*} \mathrm{HF}_{2} \cong \mathbb{F}_{2}\left[\xi_{1}, \xi_{2}, \ldots\right]$, this operation is defined on the element $\xi_{1}^{2} \in H_{2}\left(\mathrm{HF}_{2}\right)$ and, mod decomposables, unambiguously takes the value $\xi_{5} \in H_{31}\left(\mathrm{HF}_{2}\right)$.

With this theorem, we can exclude the existence of an $E_{\infty}$-algebra structure on the 2-local Brown-Peterson spectrum and several related objects (e.g. the generalized $B P\langle k\rangle$ whose cohomology is discussed in $[\mathrm{LN} 14,4.3]$ and whose additive uniqueness is discussed in [AL17]).

Theorem 1.1.2 (5.4.6, 5.5.4). Suppose that $R$ is a connective $E_{12}$-algebra with a ring homomorphism $\pi_{0} R \rightarrow \mathbb{F}_{2}$ such that the induced map on mod-2 homology $H_{*} R \rightarrow$ $H_{*} \mathrm{HF}_{2}$ is injective in degrees 5 through 13 . If $\xi_{1}^{2}$ is in the image of $H_{2}(R)$, then the element $\xi_{5}$ is in the image of $H_{31} R$ mod decomposables.

In particular, the 2-local Brown-Peterson spectrum BP, the (generalized) truncated Brown-Peterson spectra $B P\langle k\rangle$ for $k \geq 4$, and their 2-adic completions do not admit the structure of $E_{n}$-algebras for any $12 \leq n \leq \infty$.

\subsection{Remarks on obstruction theory}

The secondary operation we will define is determined by a relation between DyerLashof operations (the full relation is rather large, and is displayed in Proposition 5.4.1). For us, this relation is not obvious; it is not obvious that this particular relation is relevant; and it is not obvious that the resulting secondary operation is calculable. We did not find this relation by trial and error-or, more accurately, we tried to find relevant secondary operations by trial and error and failed. All of our preliminary attempts resulted in combinations that were excluded by necessity of compatibility with the 
Steenrod operations. In this section we will indicate a little about how the main result of this paper was found, as opposed to how it is written. ${ }^{3}$

The obstruction theory of Goerss-Hopkins [GH] takes as input a simplicial operad, an appropriate homology theory $E_{*}$, and an algebra $A$ for this simplicial operad in $E_{*} E$-comodules. From this, it produces an obstruction theory to calculate the moduli space of algebras over the geometric realization of this operad whose $E$-homology is $A$. Senger specialized this to the case where $E$ is mod- $p$ homology and the operad is a constant $E_{\infty}$-operad [Sen]. His work produced an obstruction theory whose input is an algebra $A$ with Steenrod operations and Dyer-Lashof operations satisfying instability relations and Nishida relations, whose obstruction groups are Ext-groups in this category, and which calculated the moduli space of $E_{\infty}$-algebras whose mod$p$ homology is $A$. He also developed several tools for reducing these calculations to more tractable Ext-groups that could, in the case of $B P$ or $B P\langle n\rangle$, be calculated with a Koszul resolution [Pri70]. By construction, this obstruction theory remembers that the Nishida relations will exclude a number of possible obstructions. (The problem encountered in Kriz's preprint [Kri95] could be viewed as the accidental exclusion of too many obstructions in this fashion.)

In the case of the 2-primary Brown-Peterson spectrum, calculations with this obstruction theory indicated two first potential nonzero obstruction classes. We can define $y, R^{n}$, and $v_{m}$ to be, respectively, Ext-Koszul dual to the generator $\xi_{1}^{2} \in H_{2}(B P)$, the Dyer-Lashof operation $Q^{n-1}$, and the Milnor primitive $Q_{m-1}$ in the Steenrod algebra. (The $R^{n}$ are closely related to unpublished work of Basterra-Mandell on operations in TAQ-cohomology.) Then, using this notation, the first possible obstruction classes are $v_{3}^{2} R^{19} R^{9} y$ and $v_{4} R^{21} R^{9} y$. Under the yoga of secondary operations described by Adams [Ada66], the potential obstruction class $v_{4} R^{21} R^{9} y$ would detect an obstruction from a secondary operation whose value involved $\xi_{5}$ (detected by the Milnor primitive), combining relations that (at least) involved the Adem relations for $Q^{20} Q^{8}$ and an identity satisfied by $Q^{8} \xi_{1}^{2}$.

Indeed, our main result is that this is the case. However, much of the progress in this paper traces its origin back to the actual calculation of these relations. After determining the needed identities in Proposition 5.4.1, we could identify most of the relations in $H_{*} B P \subset H_{*} \mathrm{HF}_{2}$ as already holding true in $H_{*} M U$, making it possible to begin juggling this secondary operation through much simpler ones passing through $H_{*} M U$.

\subsection{Further questions}

In the original version of this paper, we expressed the following strong belief that the 2-primary Brown-Peterson spectrum was not unique in failing to admit an $E_{\infty}$ algebra structure.

Conjecture 1.3.1. For any odd prime $p$, the p-local Brown-Peterson spectrum does not admit the structure of an $E_{\infty}$-algebra.

Senger has already extended the methods of this paper to prove Conjecture 1.3.1,

\footnotetext{
${ }^{3} \mathrm{~A}$ more detailed explanation of this calculation is now in [Law17].
} 
showing that $B P$ (and $B P\langle n\rangle$ for $n \geq 4$ ) do not admit the structure of $E_{2\left(p^{2}+2\right)}$-algebras at any prime $p$ [Sen17].

Our keystone computation in this paper is a Dyer-Lashof operation in a version of the 2-primary dual Steenrod algebra for $M U$-modules.

Problem 1.3.2. Determine how the Dyer-Lashof operations act on the p-primary MUdual Steenrod algebras $\pi_{*}\left(\mathrm{HF}_{p} \wedge_{M U} \mathrm{HF}_{p}\right)$.

Baker has shown in [Bak15] how to derive the Nishida relations, describing the interaction between cohomology operations and Dyer-Lashof operations, from the Dyer-Lashof operations in the ordinary dual Steenrod algebra. This suggests that a solution to the previous problem would give additional constraints on $M U$-algebras by describing additional relations that have to hold in their mod- $p$ homology relative to $M U$.

Problem 1.3.3. Determine analogues of Nishida relations between the homology operations on $H_{*}^{M U} R=\pi_{*}\left(\mathrm{HF}_{p} \wedge_{M U} R\right)$ and the Dyer-Lashof operations.

In particular, Remark 4.4.7 describes how the Dyer-Lashof operation that we have calculated seem to place a cap on multiplicative structure for the map $M U \rightarrow B P$ at the prime 2-a stronger cap than the one we have shown for the amount of multiplicative structure on $B P$.

Problem 1.3.4. Find constraints on the values of $n$ for which the p-local Brown-Peterson spectrum can admit the structure of an $E_{n} M U$-algebra.

Again, in the time since we raised this question, Senger has shown that $B P$ does not admit the structure of an $E_{2 p+3} M U$-algebra at any prime $p$ [Sen17].

The calculations of this paper deduce our unexpected Dyer-Lashof operation in the $M U$-dual Steenrod algebra from a multiplicative Dyer-Lashof operation in the Hopf ring for $M U$, and an induced operation in the homology of the space $S L_{1}(M U) \subset$ $G L_{1}(M U)$ of strict units. This is a first step towards determining the homology of the spectrum $g l_{1}(M U)$, about which very little is known, using the Miller spectral sequence [Mil78].

Problem 1.3.5. Determine multiplicative Dyer-Lashof operations in the Hopf ring for $M U$ and in the homology of $G L_{1}(M U)$. Determine homology groups of the unit spectrum $g l_{1}(M U)$ and the Picard spectrum pic $(M U)$, as well as information about their homotopy types.

Remark 2.1.9 points out that our description of secondary operations and Toda brackets is not optimal. For example, it sometimes requires strict basepoints for mapping spaces, strict unitality, and strict initial and terminal objects, all of which are not invariant under unbased homotopy equivalences between objects and not invariant under Dwyer-Kan equivalences between topological categories. However, the tools should apply in much wider generality; investigations in this direction have been carried out by Bhattacharya and Hank.

Problem 1.3.6. Develop a homotopical framework for secondary operations. 
For example, a combinatorial framework analogous to quasicategories that encodes the notion of a category enriched in based spaces, equivalent to that introduced by Gepner-Haugseng [GH15], would be extremely useful in this direction. Ideally, this should make Cohen-Jones-Segal's construction of filtered spectra from coherent chain complex objects [CJS95, §5] part of an equivalence between filtered objects and coherent chain complex objects in a stable $\infty$-category, extending Lurie's version of the Dold-Kan correspondence [Lur17, 1.2.4].

Our calculations with power operations in the Hopf ring make use of the $H_{\infty}^{2}$ algebra structure on $M U$, a concept from [BMMS86] that has been largely neglected in the modern literature. It should be possible to describe a fully coherent version of this structure using the language of Picard spaces and Picard spectra [MS16].

Problem 1.3.7. Give a systematic development of $E_{\infty}^{d}$-algebras as homotopy coherent versions of $H_{\infty}^{d}$-ring spectra, and show that the $H_{\infty}^{d}$-structures on classical Thom spectra constructed in [BMMS86, VIII.5.1] lift to $E_{\infty}^{d}$-algebra structures.

An $E_{\infty}^{d}$-structure on an $E_{\infty}$-algebra $R$ should be a lift of the map of spaces

$$
d \mathbb{Z} \subset \mathbb{Z} \subset \operatorname{Pic}(\mathbb{S}) \rightarrow \operatorname{Pic}(R)
$$

to a map of $E_{\infty}$-spaces, corresponding to a functor of symmetric monoidal $\infty$-categories. In close analogy with the work of Ando-Blumberg-Gepner-Hopkins-Rezk [ $\left.\mathrm{ABG}^{+} 14\right]$, the point $\{d k\} \hookrightarrow \operatorname{Pic}(\mathbb{S})$ representing $S^{d k}$ gives rise (via the $E_{\infty}$-space structure) to a diagram $B \Sigma_{m} \rightarrow \operatorname{Pic}(\mathbb{S})$, whose homotopy colimit is a Thom spectrum on $B \Sigma_{m}$ representing the extended power construction on $S^{d k}$. An $E_{\infty}^{d}$-structure on $R$ should then make the resulting diagram $B \Sigma_{m} \rightarrow \operatorname{Pic}(R)$ factor through a constant diagram with value $S^{d k m}$, allowing us to conclude that the smash product of $R$ with the Thom spectrum has an equivalence to $R \wedge\left(B \Sigma_{m}\right)_{+} \wedge S^{d k m}$.

\subsection{Outline of proof}

We will begin by calculating a Dyer-Lashof operation in the $M U$-dual Steenrod algebra $\pi_{*}\left(H \wedge_{M U} H\right)$, where $H$ is the Eilenberg-Mac Lane spectrum $\mathrm{HF}_{2}$. This has maps in from the dual Steenrod algebra $\pi_{*}(H \wedge H)$ and out to the homology of $S U$ which become a left exact sequence

$$
0 \rightarrow Q \pi_{*}(H \wedge H) \rightarrow Q \pi_{*}(H \underset{M U}{\wedge} H) \rightarrow Q H_{*} S U
$$

on indecomposables. The Dyer-Lashof operations on the left are known by work of Steinberger, and were employed by Tilson [Til16] to calculate operations in the middle term. The Dyer-Lashof operations on the right are known by work of Kochman. The operation we will calculate is the first possible hidden extension and it turns out to be nontrivial.

To carry out this calculation we rely on calculations of unstable multiplicative Dyer-Lashof operations. This uses Ravenel-Wilson's description of Hopf ring structure on the homology of the spaces in the $\Omega$-spectrum for $M U$ [RW74] and a comparison between Dyer-Lashof operations and the tom Dieck-Quillen power operations 
in $M U$-cohomology. The relevant portion of this extension is ultimately determined by the calculation of Johnson-Noel discussed earlier [JN10]. We will make extensive use of the results of Bruner-May-McClure-Steinberger in doing this calculation [BMMS86].

We will then give an alternative description of this operation in the $M U$-dual Steenrod algebra as a Dyer-Lashof operation applied to the result of a secondary operation. This allows us to use juggling formulas for secondary operations to determine a more complicated secondary operation in the dual Steenrod algebra, showing that $H_{*} B P$ is not closed under secondary operations. (We are fortunate in this regard that most of our calculations can be carried out mod decomposable elements.) In order to work with this we will describe a framework for secondary operations in Section 2 based on Harper's book [Har02], with our emphasis shifted from suspension and loop operators to loops inside mapping spaces.

\subsection{Terminology}

The notation Map always denotes a space, or simplicial set, of maps. We will refer to a diagram as homotopy commutative if it commutes in the homotopy category, and homotopy coherent if we have further chosen compatible homotopies and higher homotopies to recover a coherent diagram [Vog73, Lur09].

We will adhere to the standard conventions for function composition and path composition, even though they make no sense. Maps in a category are written using arrows $X \rightarrow Y$, and given $f: X \rightarrow Y$ and $g: Y \rightarrow Z$ there is a composite $g f$. Paths in a space are written using arrows $p \Rightarrow q$, and given $h: p \Rightarrow q$ and $k: q \Rightarrow r$ there is a path composite $h \cdot k$.

Throughout this paper, we will write $H_{*}(X ; R)$ for the homology groups of $X$ with coefficients in $R$, and similarly for cohomology. If $R$ is not specified, we view these as being taken with coefficients in a fixed finite field $\mathbb{F}_{p}$ of prime order. Homology and cohomology groups of spaces are unreduced unless otherwise specified.

When $X$ is a spectrum, $\pi_{n}(X)$ always denotes the set of maps $S^{n} \rightarrow X$ in the stable homotopy category.

We will let $M U$ be the complex cobordism spectrum and $F$ be the formal group law of $M U$, writing it as $F(x, y)=x+_{F} y=\sum a_{i, j} x^{i} y^{j}$ with $a_{i, j} \in \pi_{2(i+j-1)} M U$.

\subsection{Framework}

We are in the position that we require tools from both classical and modern frameworks.

In Section 2, we will require highly structured categories of algebras, well-behaved adjunctions between them, relative smash products, and the like. To our knowledge, the only literature that accommodate our needs for $E_{n}$-algebras is due to ElmendorffMandell [EM06], which works in the category of symmetric spectra of with the positive stable model structure [HSS00, MMSS01]. We will use the term commutative ring spectrum for a commutative monoid in symmetric spectra, and the term $E_{n}$-algebra for an algebra over a fixed $E_{n}$-operad in simplicial sets-for this it is convenient to use the $E_{\infty}$-operad of Barratt-Eccles [BE74] with its filtration by $E_{n}$-suboperads due 
to Berger [Ber97]. In this framework, Elmendorf-Mandell show that each category of $E_{n}$-algebras is a simplicial model category. For $m \leq n$, the forgetful functors from $E_{n^{-}}$ algebras to $E_{m}$-algebras or to symmetric spectra are right Quillen functors, and there is a Quillen equivalence between $E_{\infty}$-algebras and commutative ring spectra [EM06, $1.3,1.4]$.

In Section 3 and beyond, where we are calculating with $M U$ and the dual Steenrod algebra, we require classical results: particularly results of Cohen-Lada-May [CLM76], May-Quinn-Ray [May77], Bruner-May-McClure-Steinberger [BMMS86], and Ravenel-Wilson [RW74]. All of these results rest on the interaction between a (possibly highly structured) ring spectrum $E$ and the spaces $E_{n}$ in an $\Omega$-spectrum representing it, an item not immediately available in the positive stable model structure. Most of these references use more classical categories of spectra, such as those from [LMSM86]. In particular, comparisons are easiest to draw to the $\mathbb{S}$-modules of [EKMM97], and these all have homotopically equivalent notions of commutative ring spectra as shown in [MMSS01]. This gives us a path to show that operations and relations between them that we construct in Section 2 can be related to our calculations. (We do not mean to assert that the constructions in Section 2 cannot be carried out within $\mathbb{S}$-modules. To our knowledge, ours is the shortest path without the hard work involved in creating an equivalent of [EM06].)

\subsection{Acknowledgements}

The author has benefited from discussions and perspectives provided by many people in the course of developing this paper. The author would particularly like to thank Andrew Baker, Clark Barwick, David Benson, Andrew Blumberg, Robert Bruner, John R. Harper, Fabien Hebestreit, Mike Hill, Paul Goerss, Weinan Lin, Michael Mandell, Peter May, Haynes Miller, Ulrich Pennig, Charles Rezk, Andrew Senger, Neil Strickland, Markus Szymik, Sean Tilson, Craig Westerland, Dylan Wilson, and Steven Wilson for their assistance. The anonymous referee also provided a great deal of useful feedback on this paper.

\section{Secondary operations}

A secondary composite is the first basic type of obstruction encountered when lifting a homotopy commutative diagram to a homotopy coherent diagram.

Definition 2.0.1. Let $\mathcal{D}$ be a category enriched in spaces. Suppose that we are given the following data:

1. a sequence $\left(X_{0}, X_{1}, X_{2}, X_{3}\right)$ of objects of $\mathcal{D}$,

2. maps $f_{i j}: X_{i} \rightarrow X_{j}$ for $i<j$, and

3. paths $h_{i j k}: f_{j k} f_{i j} \Rightarrow f_{i k}$ in $\operatorname{Map}_{\mathcal{D}}\left(X_{i}, X_{k}\right)$ for $i<j<k$.

Then the associated secondary composite is the element of $\pi_{1}\left(\operatorname{Map}_{\mathcal{D}}\left(X_{0}, X_{3}\right), f_{03}\right)$ represented by the path composite

$$
\left(h_{023}\right)^{-1} \cdot\left(f_{23} h_{012}\right)^{-1} \cdot\left(h_{123} f_{01}\right) \cdot h_{013},
$$


viewed as a loop based at $f_{03}$.

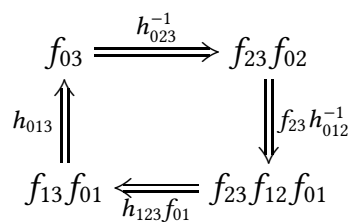

(In Remark 2.1.9 we will discuss a quasicategorical expression of this data.)

In the following sections we will describe secondary composites which are comparable with Massey products or Toda brackets; they rely on the existence of distinguished "null" maps so that we can make sense of composites being trivial. Our perspective is based on Harper's book [Har02].

\subsection{Secondary operations and brackets}

Throughout this section, let $C$ be a category enriched in pointed spaces (or, with appropriate modifications, pointed simplicial sets) under $\wedge$, and write $\operatorname{Map}_{C}(x, y)$ for the mapping space between any pair of objects of $C$. We refer to the basepoint of this mapping space as the null map or $*$; null maps satisfy $f *=* f=*$ for any $f .4$

Definition 2.1.1. Suppose we have maps

$$
X_{0} \stackrel{f}{\rightarrow} X_{1} \stackrel{g}{\rightarrow} X_{2}
$$

in $C$. A tethering of this composite is a homotopy class of nullhomotopy of $g f:$ a homotopy class of path $h: g f \Rightarrow *$ in $\operatorname{Map}_{C}\left(X_{0}, X_{2}\right)$ (cf. [Har02, 4.1.2]). We will write $g \stackrel{h}{\leftrightarrow} f$ to indicate such a tethering, and $g \leftrightarrow f$ to indicate that there is a chosen tethering which is either implicit or not important to name.

Remark 2.1.2. If a triple composite $k g f$ is nullhomotopic, then a tethering $k g \stackrel{h}{\leftrightarrow} f$ is the same data as a tethering $k \stackrel{h}{h} g f$.

Definition 2.1.3. Suppose we have maps

$$
X_{0} \stackrel{f_{01}}{\longrightarrow} X_{1} \stackrel{f_{12}}{\longrightarrow} X_{2} \stackrel{f_{23}}{\longrightarrow} X_{3},
$$

and tetherings $f_{23} \stackrel{h_{123}}{\leftrightarrow} f_{12} \stackrel{h_{012}}{\leftrightarrow} f_{01}$. Then we define the element

$$
\left\langle f_{23} \stackrel{h_{123}}{\leftrightarrow} f_{12} \stackrel{h_{012}}{\leftrightarrow} f_{01}\right\rangle \in \pi_{1}\left(\operatorname{Map}_{C}\left(X_{0}, X_{3}\right), *\right)
$$

to be the path composite $\left(f_{23} h_{012}\right)^{-1} \cdot h_{123} f_{01}$ obtained by gluing together the two nullhomotopies $f_{23} f_{12} f_{01} \Rightarrow *$. This is the secondary composite, as in Definition 2.0.1, obtained by choosing $f_{02}=f_{03}=f_{13}=*$ and the trivial nullhomotopies $h_{013}$ and $h_{023}$.

${ }^{4}$ Strictly speaking, the smash product on pointed spaces is nonassociative and so does not give rise to a monoidal category [MS, §1.7]. We really mean that we are working in an appropriate "convenient category," such as compactly generated spaces. 
Definition 2.1.4. Suppose we have maps

$$
X_{0} \stackrel{f_{01}}{\longrightarrow} X_{1} \stackrel{f_{12}}{\longrightarrow} X_{2} \stackrel{f_{23}}{\longrightarrow} X_{3} .
$$

If we have chosen a tethering $f_{23} \stackrel{h}{\leftrightarrow} f_{12}$ and $f_{12} f_{01}$ is nullhomotopic, we write

$$
\left\langle f_{23} \stackrel{h}{\rightsquigarrow} f_{12}, f_{01}\right\rangle \subset \pi_{1}\left(\operatorname{Map}_{C}\left(X_{0}, X_{3}\right), *\right)
$$

for the set of all elements $\left\langle f_{23} \stackrel{h}{\leftrightarrow} f_{12} \stackrel{k}{\leftrightarrow} f_{01}\right\rangle$ as $k$ ranges over possible tetherings, and refer to $\left\langle f_{23} \stackrel{h}{\leftrightarrow} f_{12},-\right\rangle$ as the secondary operation determined by the tethering. The set of maps $f_{01}$ such that $f_{12} f_{01}$ is nullhomotopic is referred to as the domain of definition of this secondary operation, and the possibly multivalued nature of this function as the indeterminacy of the secondary operation.

The secondary operations $\left\langle-, f_{12} \leadsto f_{01}\right\rangle$ are defined in the same way.

Definition 2.1.5. Suppose we have maps

$$
X_{0} \stackrel{f_{01}}{\longrightarrow} X_{1} \stackrel{f_{12}}{\longrightarrow} X_{2} \stackrel{f_{23}}{\longrightarrow} X_{3}
$$

such that the double composites $f_{23} f_{12}$ and $f_{12} f_{01}$ are nullhomotopic. We define the subset

$$
\left\langle f_{23}, f_{12}, f_{01}\right\rangle \subset \pi_{1}\left(\operatorname{Map}_{C}\left(X_{0}, X_{3}\right), *\right),
$$

or bracket, to be the set of all secondary composites $\left\langle f_{23} \leadsto f_{12} \leadsto f_{01}\right\rangle$.

Proposition 2.1.6. Changing the tethering and homotopy class of maps alters the value of a secondary composite by multiplication by loops, as follows. If $f_{23}$ is homotopic to $f_{23}^{\prime}$, we have

$$
\left\langle f_{23} \stackrel{h}{\leftrightarrow} f_{12} \stackrel{k}{\leftrightarrow} f_{01}\right\rangle=\left\langle f_{23}^{\prime} \stackrel{h^{\prime}}{\leftrightarrow} f_{12} \stackrel{k}{\leftrightarrow} f_{01}\right\rangle \cdot\left(u f_{01}\right)
$$

for some $u \in \pi_{1} \operatorname{Map}_{C}\left(X_{1}, X_{3}\right)$ that is determined by $h, h^{\prime}$, and a homotopy between $f_{23}$ and $f_{23}^{\prime}$. Similarly, if $f_{01}$ is homotopic to $f_{01}^{\prime}$, we have

$$
\left\langle f_{23} \stackrel{h}{\leftrightarrow} f_{12} \stackrel{k}{\leftrightarrow} f_{01}\right\rangle=\left(f_{23} v\right) \cdot\left\langle f_{23} \stackrel{h}{\leftrightarrow} f_{12} \stackrel{k^{\prime}}{\leftrightarrow} f_{01}^{\prime}\right\rangle
$$

for some $v \in \pi_{1} \operatorname{Map}_{C}\left(X_{0}, X_{2}\right)$.

If we replace all three maps with homotopic maps and choose new tetherings, we have

$$
\left\langle f_{23} \stackrel{h}{\leftrightarrow} f_{12} \stackrel{k}{\leftrightarrow} f_{01}\right\rangle=\left(f_{23} v\right) \cdot\left\langle f_{23}^{\prime} \stackrel{h^{\prime}}{\leftrightarrow} f_{12}^{\prime} \stackrel{k^{\prime}}{\leftrightarrow} f_{01}^{\prime}\right\rangle \cdot\left(u f_{01}\right)
$$

for some $u \in \pi_{1} \operatorname{Map}_{C}\left(X_{1}, X_{3}\right)$ and $v \in \pi_{1} \operatorname{Map}_{C}\left(X_{0}, X_{2}\right)$.

In particular, this describes completely the indeterminacy in secondary operations and brackets, and shows that (up to this indeterminacy) a secondary operation or a bracket is well-defined on homotopy classes of maps. 
Proof. We will prove the first identification, as the second is symmetric. Since $f_{23}$ and $f_{23}^{\prime}$ are homotopic, there is a path $j: f_{23} \Rightarrow f_{23}^{\prime}$ in $\operatorname{Map}_{C}\left(X_{2}, X_{3}\right)$. The composition

$$
j k: \Delta^{1} \times \Delta^{1} \stackrel{j \times k}{\longrightarrow} \operatorname{Map}_{C}\left(X_{2}, X_{3}\right) \times \operatorname{Map}_{C}\left(X_{0}, X_{2}\right) \rightarrow \operatorname{Map}_{C}\left(X_{0}, X_{3}\right)
$$

determines a homotopy from $f_{23} k$ to $\left(j f_{12} f_{01}\right) \cdot\left(f_{23}^{\prime} k\right)$, making them equal in the fundamental groupoid of $\operatorname{Map}_{C}\left(X_{0}, X_{3}\right)$.

In this fundamental groupoid, we then have the following sequence of identities:

$$
\begin{aligned}
& \left\langle f_{23} \stackrel{h}{\leftrightarrow} f_{12} \stackrel{k}{\leftrightarrow} f_{01}\right\rangle=\left(f_{23} k\right)^{-1} \cdot\left(h f_{01}\right) \\
& =\left(f_{23}^{\prime} k\right)^{-1} \cdot\left(j f_{12} f_{01}\right)^{-1} \cdot\left(h f_{01}\right) \\
& =\left(f_{23}^{\prime} k\right)^{-1} \cdot\left(h^{\prime} f_{01}\right) \cdot\left(h^{\prime} f_{01}\right)^{-1} \cdot\left(j f_{12} f_{01}\right)^{-1} \cdot\left(h f_{01}\right) \\
& =\left\langle f_{23}^{\prime} \stackrel{h^{\prime}}{\leftrightarrow} f_{12} \stackrel{k}{\leftrightarrow} f_{01}\right\rangle \cdot\left[\left(j f_{12} \cdot h^{\prime}\right)^{-1} \cdot h\right] f_{01}
\end{aligned}
$$

Letting $u=\left(j f_{12} \cdot h^{\prime}\right)^{-1} \cdot h \in \pi_{1} \operatorname{Map}_{C}\left(X_{0}, X_{2}\right)$ gives the desired result.

Corollary 2.1.7. A secondary operation $\left\langle f_{23} \stackrel{h}{\leftrightarrow} f_{12},-\right\rangle$ determines a well-defined map $\Phi$ on $\operatorname{ker} f_{12} \subset \pi_{0} \operatorname{Map}_{C}\left(X_{0}, X_{1}\right)$ whose values are right cosets:

$$
\operatorname{ker} f_{12} \stackrel{\Phi}{\rightarrow}\left(f_{23} \pi_{1} \operatorname{Map}_{C}\left(X_{0}, X_{2}\right)\right) \backslash \pi_{1} \operatorname{Map}_{C}\left(X_{0}, X_{3}\right) .
$$

If two tetherings $h, h^{\prime}$ give rise to operations $\Phi, \Phi^{\prime}$, then there exists an element $u \in$ $\pi_{1} \operatorname{Map}_{C}\left(X_{1}, X_{3}\right)$ such that

$$
\Phi x=\Phi^{\prime} x \cdot(u x)
$$

for all $x \in \operatorname{ker} f_{12} \subset \pi_{0} \operatorname{Map}_{C}\left(X_{0}, X_{1}\right)$.

Dual results hold for $\left\langle-, f_{12} \leadsto f_{01}\right\rangle$.

Corollary 2.1.8. Suppose we have maps

$$
X_{0} \stackrel{f_{01}}{\longrightarrow} X_{1} \stackrel{f_{12}}{\longrightarrow} X_{2} \stackrel{f_{23}}{\longrightarrow} X_{3}
$$

such that the double composites $f_{23} f_{12}$ and $f_{12} f_{01}$ are nullhomotopic. Then the bracket $\left\langle f_{23}, f_{12}, f_{01}\right\rangle$ depends only on the homotopy classes of $f_{i, i+1}$ and is a well-defined double coset in

$$
\left(f_{23} \pi_{1} \operatorname{Map}_{C}\left(X_{0}, X_{2}\right)\right) \mid \pi_{1} \operatorname{Map}_{C}\left(X_{0}, X_{3}\right) /\left(\pi_{1} \operatorname{Map}_{C}\left(X_{1}, X_{3}\right) f_{01}\right) .
$$

Remark 2.1.9. A more flexible version of the above constructions should exist, where basepoints are replaced by some appropriate system of maps $E_{i, j} \rightarrow \operatorname{Map}_{C}\left(X_{i}, X_{j}\right)$ from contractible spaces $E_{i, j}$, together with appropriate lifts of the composition maps. For example, the category of diagrams of spaces $E \rightarrow X$ is a monoidal category under the pushout-product, and so we could ask for $C$ to be enriched in this category with the constraint that the space $E$ is always contractible. We might instead try to find an appropriate analogue in terms of quasicategories satisfying certain basepoint 
conditions: in the notation of [Lur09], the data to describe a secondary composite in Definition 2.0.1 defines a map of enriched categories $\mathfrak{C}\left[\partial \Delta^{3}\right] \rightarrow \mathcal{D}$, and the secondary composite is the obstruction to extending it to a map $\mathbb{C}\left[\Delta^{3}\right] \rightarrow \mathcal{D}$ (a homotopy coherent triple composite). Both of these constructions would apply more widely, but involve more bookkeeping and possibly require a more advanced technical framework. We have elected to use constructions in categories where this will not be necessary in order to minimize the technical load.

The definitions of secondary operations and brackets are preserved in an obvious way under functors between enriched categories.

Proposition 2.1.10. Suppose $F: C \rightarrow C^{\prime}$ is an enriched functor between categories enriched in pointed spaces. Then any tethering $g \stackrel{h}{\leftrightarrow} f$ in $C$ induces a tethering $F g \stackrel{F h}{\leftrightarrow} F f$ in $C^{\prime}$. We have an equality

$$
F\left(\left\langle f_{23} \stackrel{h}{\leftrightarrow} f_{12} \stackrel{k}{\leftrightarrow} f_{01}\right\rangle\right)=\left\langle F f_{23} \stackrel{F h}{\leftrightarrow} F f_{12} \stackrel{F k}{\leftrightarrow} F f_{01}\right\rangle,
$$

and we have containments as follows:

$$
\begin{aligned}
& F\left(\left\langle f_{23} \stackrel{h}{\leftrightarrow} f_{12}, f_{01}\right\rangle\right) \subset\left\langle F f_{23} \stackrel{F h}{\leftrightarrow} F f_{12}, F f_{01}\right\rangle \\
& F\left(\left\langle f_{23}, f_{12} \stackrel{k}{\leftrightarrow} f_{01}\right\rangle\right) \subset\left\langle F f_{23}, F f_{12} \stackrel{F k}{\leftrightarrow} F f_{01}\right\rangle \\
& F\left(\left\langle f_{23}, f_{12}, f_{01}\right\rangle\right) \subset\left\langle F f_{23}, F f_{12}, F f_{01}\right\rangle
\end{aligned}
$$

There is a further extension in the case where we have an enriched adjunction. An example of such a result appears below.

Proposition 2.1.11. Suppose that we have an enriched adjoints $F: C \rightarrow \mathcal{D}$ and $G: \mathcal{D} \rightarrow C$, encoded by a natural based homeomorphism

$$
\theta: \operatorname{Map}_{C}(X, G Y) \cong \operatorname{Map}_{C}(F X, Y) .
$$

Given maps

$$
X_{0} \stackrel{f_{01}}{\longrightarrow} X_{1} \stackrel{f_{12}}{\longrightarrow} X_{2} \stackrel{g}{\rightarrow} G Y
$$

and tetherings

$$
g \stackrel{h}{\leftrightarrow} f_{12} \stackrel{k}{\leftrightarrow} f_{01},
$$

the map $\theta$ induces an identity

$$
\theta_{*}\left\langle g \stackrel{h}{\leftrightarrow} f_{12} \stackrel{k}{\leftrightarrow} f_{01}\right\rangle=\left\langle\theta g \stackrel{\theta h}{\leftrightarrow} F f_{12} \stackrel{F k}{\leftrightarrow} F f_{01}\right\rangle .
$$

Corollary 2.1.12. There are containments

$$
\theta_{*}\left\langle g, f_{12} \stackrel{k}{\leftrightarrow} f_{01}\right\rangle \subset\left\langle\theta g, F f_{12} \stackrel{F k}{\leftrightarrow} F f_{01}\right\rangle
$$

and

$$
\theta_{*}\left\langle g, f_{12}, f_{01}\right\rangle \subset\left\langle\theta g, F f_{12}, F f_{01}\right\rangle .
$$




\subsection{Pointings and augmentations}

In this section we let $\mathcal{D}$ be a category enriched in spaces (now assumed to have no basepoint). In this section we indicate a construction that replaces $\mathcal{D}$ with a category enriched in pointed spaces.

Definition 2.2.1. An augmented object of $\mathcal{D}$ is an object $X \in \mathcal{D}$ equipped with a map $Y \rightarrow \emptyset$ to an initial object of $\mathcal{D}$. The space of maps between two augmented objects is the subspace of ordinary maps that commute with the augmentations.

A pointed object of $\mathcal{D}$ is an object $Z \in \mathcal{D}$ equipped with a map $* \rightarrow Z$ from a terminal object of $\mathcal{D}$. The space of maps between two pointed objects is the subspace of ordinary maps that commute with the pointings.

Definition 2.2.2. Suppose $\mathcal{D}$ is a category enriched in spaces. We define $\mathcal{D}^{ \pm}$, the category of possibly pointed or augmented objects of $\mathcal{D}$, to be the following category enriched in based spaces.

An object of $\mathcal{D}^{ \pm}$is one of three types:

1. an augmented object $X \rightarrow \emptyset$ of $\mathcal{D}$,

2. an ordinary object $Y$ of $\mathcal{D}$, or

3. a pointed object $* \rightarrow Z$ of $\mathcal{D}$.

The mapping spaces in $\mathcal{D}^{ \pm}$are given as follows.

1. The space of maps between two augmented objects $X \rightarrow \emptyset, X^{\prime} \rightarrow \emptyset^{\prime}$ is the space of maps of augmented objects, with basepoint given by the composite $X \rightarrow \emptyset \rightarrow X^{\prime}$.

2. The space of maps between two pointed objects $* \rightarrow Z, *^{\prime} \rightarrow Z^{\prime}$ is the space of maps of pointed objects, with basepoint given by the composite $Z \rightarrow *^{\prime} \rightarrow Z^{\prime}$.

3. The space of maps between two ordinary objects $Y, Y^{\prime}$ is the based $\operatorname{space}_{\operatorname{Map}_{\mathcal{D}}}\left(Y, Y^{\prime}\right)_{+}$, whose disjoint basepoint is called the formal null map.

4. The space of maps from an augmented object $X \rightarrow \emptyset$ to an ordinary object $Y$ is the space of maps $X \rightarrow Y$, with basepoint given by the map $X \rightarrow \emptyset \rightarrow Y$.

5. The space of maps from an ordinary object $Y$ to a pointed object $* \rightarrow Z$ is the space of maps $Y \rightarrow Z$, with basepoint given by the map $Y \rightarrow * \rightarrow Z$.

6. The space of maps from an augmented object $X \rightarrow \emptyset$ to a pointed object $* \rightarrow$ $Z$ is the space of maps $X \rightarrow Z$, with basepoint given by the canonical map factoring through either $\emptyset$ or $*$ in the commutative diagram

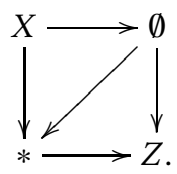


7. All other mapping spaces are one-point spaces-there are no non-basepoint maps from ordinary objects to augmented ones, or from pointed objects to ordinary ones. We also refer to these as formal null maps.

We have full subcategories of $\mathcal{D}^{ \pm}$spanned by fewer than all three of these types of objects: for example, we have the categories of augmented objects, pointed objects, possibly augmented objects, and possibly pointed objects of $\mathcal{D}$.

Proposition 2.2.3. The category $\mathcal{D}^{ \pm}$is enriched in pointed spaces under $\wedge$.

In $\mathcal{D}^{ \pm}$, if a composite $X \rightarrow Y \rightarrow Z$ is nullhomotopic then $X$ is augmented, $Z$ is pointed, or one of the maps is a formal null map (in which case there is a canonical tethering).

This construction makes it possible to take a category $\mathcal{D}$ and sensibly talk about secondary operations and brackets for a composite $X_{0} \rightarrow X_{1} \rightarrow X_{2} \rightarrow X_{3}$ in $\mathcal{D}$ if the first map is a map of augmented objects, if the last map is a map of pointed objects, or if the first object is augmented and the last object is pointed. (If the maps arise from $\mathcal{D}$ then a formal null map cannot appear.)

Example 2.2.4. If $C$ has homotopy pushouts and we have augmented objects $X_{0} \rightarrow$ $X_{1} \rightarrow \emptyset$, the bracket can be identified with an element in $\pi_{0} \operatorname{Map}_{C}\left(\Sigma X_{0}, X_{3}\right)$, represented by the outside rectangle in the homotopy coherent diagram

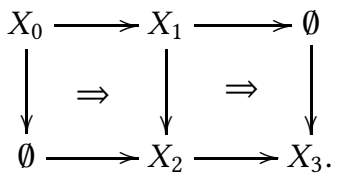

The indeterminacy in the bracket is given by path concatenation with composites of either of the following forms:

$$
\Sigma X_{0} \stackrel{v}{\longrightarrow} X_{2} \stackrel{f_{23}}{\longrightarrow} X_{3} \quad \Sigma X_{0} \stackrel{\Sigma f_{01}}{\longrightarrow} \Sigma X_{1} \stackrel{u}{\longrightarrow} X_{3}
$$

Dual results hold if we are given pointed objects $* \rightarrow X_{2} \rightarrow X_{3}$, so that the bracket can be identified with an element in $\pi_{0} \operatorname{Map}_{C}\left(X_{0}, \Omega X_{3}\right)$. To avoid grief in these identifications, especially with respect to a loop-suspension adjunction, it is important to pay attention to the orientation of $S^{1}$ as detailed at length in [Har02]. This is why we have indicated directions for 2-cells.

In the "mixed" case, there is little profound that we can say other than identification of a element in the bracket with the loop determined by a homotopy coherent diagram






\subsection{Juggling and Peterson-Stein formulas}

In this section we return to assuming that we have a category $C$ enriched in based spaces.

There are several "juggling" formulas that describe the relationship between brackets and function composition. All of them are obtained by choosing representative nullhomotopies and composing them appropriately, as in the Peterson-Stein formulas [PS59].

Lemma 2.3.1. Suppose we have a sequence of objects $\left(X_{0}, \ldots, X_{4}\right)$, together with maps $f_{i, i+1}: X_{i} \rightarrow X_{i+1}$ and tetherings

$$
f_{34} \leadsto f_{23} \leadsto f_{12} \leadsto f_{01} \text {. }
$$

Then there is an identity

$$
f_{34}\left\langle f_{23} \leadsto f_{12} \leadsto f_{01}\right\rangle^{-1}=\left\langle f_{34} \leadsto f_{23} \leadsto f_{12}\right\rangle f_{01}
$$

in $\pi_{1} \operatorname{Map}_{C}\left(X_{0}, X_{4}\right)$.

Example 2.3.2. In the case where $X_{2} \rightarrow X_{3} \rightarrow X_{4}$ are maps of pointed objects in a category $\mathcal{D}$, this Peterson-Stein relation expresses that both loops in $\operatorname{Map}_{\mathcal{D}^{ \pm}}\left(X_{0}, X_{4}\right)$ are homotopic to the loop determined by the following homotopy coherent diagram:

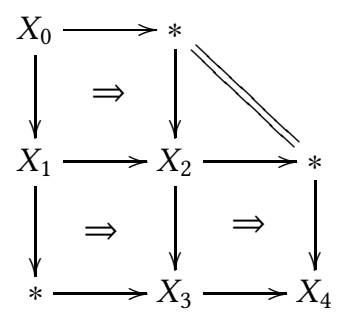

Similarly, in the mixed case we will need to derive Peterson-Stein relations from diagrams such as the following:

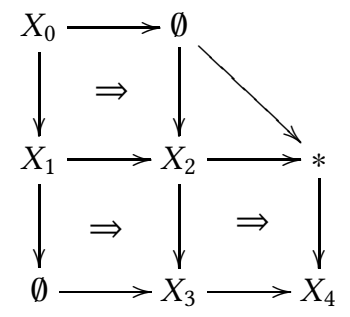

Lemma 2.3.3. Each of the following juggling formulas holds whenever defined.

$$
\begin{aligned}
& f_{34}\left\langle f_{23} \stackrel{h}{\leftrightarrow} f_{12} \stackrel{k}{\leftrightarrow} f_{01}\right\rangle=\left\langle f_{34} f_{23} \stackrel{f_{34} h}{\leftrightarrow} f_{12} \stackrel{k}{\leftrightarrow} f_{01}\right\rangle \\
& \left\langle f_{34} f_{23} \stackrel{h}{\leftrightarrow} f_{12} \stackrel{k}{\leftrightarrow} f_{01}\right\rangle=\left\langle f_{34} \stackrel{h}{\leftrightarrow} f_{23} f_{12} \stackrel{f_{23} k}{\leftrightarrow} f_{01}\right\rangle \\
& \left\langle f_{34} \stackrel{h f_{12}}{\leftrightarrow} f_{23} f_{12} \stackrel{k}{\leftrightarrow} f_{01}\right\rangle=\left\langle f_{34} \stackrel{h}{\leftrightarrow} f_{23} \stackrel{k}{\leftrightarrow} f_{12} f_{01}\right\rangle \\
& \left\langle f_{34} \stackrel{h}{\leftrightarrow} f_{23} \stackrel{k f_{01}}{\leftrightarrow} f_{12} f_{01}\right\rangle=\left\langle f_{34} \stackrel{h}{n \rightarrow} f_{23} \stackrel{k}{\leftrightarrow} f_{12}\right\rangle f_{01}
\end{aligned}
$$


As we range over possible choices of tethering, these lemmas expressing equality of secondary composites become containment relations for secondary operations and brackets.

Proposition 2.3.4. Each of the following juggling formulas for secondary operations holds whenever both sides are defined:

$$
\begin{aligned}
& \left\langle f_{4} \stackrel{\leftrightarrow}{\rightsquigarrow} f_{3}, f_{2}\right\rangle f_{1}=f_{4}\left\langle f_{3}, f_{2}, f_{1}\right\rangle^{-1} \\
& f_{4}\left\langle f_{3} \stackrel{h}{\leftrightarrow} f_{2}, f_{1}\right\rangle=\left\langle f_{4} f_{3} \stackrel{f_{4} h}{\leftrightarrow} f_{2}, f_{1}\right\rangle \\
& \left\langle f_{4} f_{3} \stackrel{h}{\leftrightarrow} f_{2}, f_{1}\right\rangle \subset\left\langle f_{4} \stackrel{h}{\leftrightarrow} f_{3} f_{2}, f_{1}\right\rangle \\
& \left\langle f_{4} \stackrel{h f_{2}}{\leftrightarrow} f_{3} f_{2}, f_{1}\right\rangle=\left\langle f_{4} \stackrel{h}{\leftrightarrow} f_{3}, f_{2} f_{1}\right\rangle \\
& \left\langle f_{4} \stackrel{h}{\leftrightarrow} f_{3}, f_{2} f_{1}\right\rangle \supset\left\langle f_{4} \stackrel{h}{\leftrightarrow} f_{3}, f_{2}\right\rangle f_{1}
\end{aligned}
$$

Dual results hold for secondary operations $\left\langle-, f_{2} \leadsto f_{1}\right\rangle$.

Proof. We will give the argument for the first statement, as the others are similar but less complex. Given fixed tetherings $f_{4} \stackrel{h}{\leftrightarrow} f_{3} \stackrel{k}{\leftrightarrow} f_{2} \stackrel{\ell}{\leftrightarrow} f_{1}$, we find that the left-hand side consists of elements of the following form:

$$
\begin{aligned}
\left\langle f_{4} \stackrel{h}{\leftrightarrow} f_{3} \stackrel{k^{\prime}}{\leftrightarrow} f_{2}\right\rangle f_{1} & =\left[\left(f_{4} v\right) \cdot\left\langle f_{2} \stackrel{h}{\leftrightarrow} f_{3} \stackrel{k}{\leftrightarrow} f_{2}\right\rangle\right] f_{1} \\
& =\left(f_{4} v f_{1}\right) \cdot\left\langle f_{2} \stackrel{h}{\leftrightarrow} f_{3} \stackrel{k}{\leftrightarrow} f_{2}\right\rangle f_{1}
\end{aligned}
$$

The right-hand side consists of elements of the following form:

$$
\begin{aligned}
f_{4}\left\langle f_{3} \stackrel{k^{\prime}}{\leftrightarrow} f_{2} \stackrel{\ell^{\prime}}{\leftrightarrow} f_{1}\right\rangle^{-1} & =f_{4}\left[\left(f_{3} w\right) \cdot\left\langle f_{2} \stackrel{h}{\leftrightarrow} f_{3} \stackrel{k}{\leftrightarrow} f_{2}\right\rangle \cdot\left(u f_{1}\right)\right]^{-1} \\
& =f_{4}\left[\left(u^{-1} f_{1}\right) \cdot\left\langle f_{2} \stackrel{h}{\leftrightarrow} f_{3} \stackrel{k}{\leftrightarrow} f_{2}\right\rangle^{-1} \cdot\left(f_{3} w^{-1}\right)\right] \\
& =\left(f_{4} u^{-1} f_{1}\right) \cdot f_{4}\left\langle f_{2} \stackrel{h}{\rightsquigarrow} f_{3} \stackrel{k}{\rightsquigarrow} f_{2}\right\rangle^{-1} \cdot\left(f_{4} f_{3} w^{-1}\right)
\end{aligned}
$$

However, $f_{4} f_{3} w^{-1}$ is always trivial because $f_{4} f_{3}$ is nullhomotopic, and so the two sets coincide by Lemma 2.3.1.

Proposition 2.3.5. Each of the following juggling formulas for brackets holds whenever both sides are defined:

$$
\begin{aligned}
& \left\langle f_{4}, f_{3}, f_{2}\right\rangle f_{1}=f_{4}\left\langle f_{3}, f_{2}, f_{1}\right\rangle^{-1} \\
& f_{4}\left\langle f_{3}, f_{2}, f_{1}\right\rangle \subset\left\langle f_{4} f_{3}, f_{2}, f_{1}\right\rangle \\
& \left\langle f_{4} f_{3}, f_{2}, f_{1}\right\rangle \subset\left\langle f_{4}, f_{3} f_{2}, f_{1}\right\rangle \\
& \left\langle f_{4}, f_{3} f_{2}, f_{1}\right\rangle \supset\left\langle f_{4}, f_{3}, f_{2} f_{1}\right\rangle \\
& \left\langle f_{4}, f_{3}, f_{2} f_{1}\right\rangle \supset\left\langle f_{4}, f_{3}, f_{2}\right\rangle f_{1}
\end{aligned}
$$


We end with a remark on adjunctions. In the presence of an (enriched) adjunction between categories $C$ and $\mathcal{D}$, we can describe relationships between secondary operations. Recall that an enriched functor $F: C \rightarrow \mathcal{D}$ with enriched left adjoint $G$ determines (and is determined by) an enriched category $\mathcal{E}$ with object set $O b(C) \cup O b(\mathcal{D})$, such that:

$$
\operatorname{Map}_{\mathcal{E}}(x, y)= \begin{cases}\operatorname{Map}_{\mathcal{C}}(x, y) & \text { if } x, y \in C \\ \operatorname{Map}_{\mathcal{D}}(x, y) & \text { if } x, y \in \mathcal{D} \\ \operatorname{Map}_{\mathcal{C}}(x, G y) \cong \operatorname{Map}_{\mathcal{D}}(F x, y) & \text { if } x \in C, y \in \mathcal{D} \\ \emptyset & \text { otherwise }\end{cases}
$$

This allows us to describe augmented and pointed objects in the presence of an adjunction and define brackets even amongst objects in categories related by adjunctions. We could, if desired, rephrase several of our constructions in these terms, in particular with respect to brackets that involve maps out of free objects.

\subsection{Additive structures}

In prominent examples, some of the mapping spaces in $C$ have natural "addition" structures.

Definition 2.4.1. An object $Y \in C$ is an $H$-object if $\operatorname{Map}_{C}(-, Y)$ naturally takes values in $H$-spaces: it is equipped with a natural homotopy-unital binary operation + whose unit is the basepoint. A map of H-objects is a map $Y \rightarrow Y^{\prime}$ preserving this structure.

An object $X \in C$ is an co-H-object if $\operatorname{Map}_{C}(X,-)$ naturally takes values in $H$-spaces: it is equipped with a natural homotopy-unital binary operation + whose unit is the basepoint. A map of co-H-objects is a map $X \rightarrow X^{\prime}$ preserving this structure.

Proposition 2.4.2. Suppose $X$ is a co-H-object in $C$ and that we have maps $f, f^{\prime}: X \rightarrow$ $Y$ and $g: Y \rightarrow Z$, together with tetherings $g \stackrel{h}{\leftrightarrow} f$ and $g \stackrel{h^{\prime}}{\leftrightarrow} f^{\prime}$. Then the pointwise product on paths in $\operatorname{Map}_{C}(X,-)$ gives a tethering $g \stackrel{h+h^{\prime}}{\leftrightarrow}\left(f+f^{\prime}\right)$.

Proposition 2.4.3. Each of the following addition formulas holds whenever both sides are defined and the source object is an co-H-object in $\mathrm{C}$ :

$$
\begin{aligned}
\left\langle f_{3} \stackrel{h}{\leftrightarrow} f_{2} \stackrel{k+k^{\prime}}{\rightsquigarrow}\left(f_{1}+f_{1}^{\prime}\right)\right\rangle & =\left\langle f_{3} \stackrel{h}{\leftrightarrow} f_{2} \stackrel{k}{\leftrightarrow} f_{1}\right\rangle+\left\langle f_{3} \stackrel{h}{\leftrightarrow} f_{2} \stackrel{k^{\prime}}{\leftrightarrow} f_{1}^{\prime}\right\rangle \\
\left\langle f_{3} \stackrel{h}{\rightsquigarrow} f_{2},\left(f_{1}+f_{1}^{\prime}\right)\right\rangle & =\left\langle f_{3} \stackrel{h}{\rightsquigarrow} f_{2}, f_{1}\right\rangle+\left\langle f_{3} \stackrel{h}{\rightsquigarrow} f_{2}, f_{1}^{\prime}\right\rangle \\
\left\langle f_{3}, f_{2},\left(f_{1}+f_{1}^{\prime}\right)\right\rangle & \subset\left\langle f_{3}, f_{2}, f_{1}\right\rangle+\left\langle f_{3}, f_{2}, f_{1}^{\prime}\right\rangle
\end{aligned}
$$

Dual results hold for $H$-objects.

Here the addition on paths is the pointwise $H$-space structure. The addition on $\pi_{1} \operatorname{Map}_{C}\left(X_{0}, X_{3}\right)$ is, by the Eckmann-Hilton argument, equivalent to either path concatenation or the pointwise $H$-space structure on paths, and makes this group abelian. 
Proof. The first identity is expressed by the following interaction between path composition and the pointwise $H$-space structure:

$$
\begin{aligned}
{\left[f_{3}\left(k+k^{\prime}\right)\right]^{-1} \cdot\left[h\left(f_{1}+f_{1}^{\prime}\right)\right] } & =\left[\left(f_{3} k\right)^{-1}+\left(f_{3} k^{\prime}\right)^{-1}\right] \cdot\left[h f_{1}+h f_{1}^{\prime}\right] \\
& =\left[\left(f_{3} k\right)^{-1} \cdot h f_{1}\right]+\left[\left(f_{3} k^{\prime}\right)^{-1} \cdot h f_{1}^{\prime}\right]
\end{aligned}
$$

Letting $k$ and $k^{\prime}$ vary over possible tetherings, this then shows that

$$
\left\langle f_{3} \stackrel{h}{\leftrightarrow} f_{2},\left(f_{1}+f_{1}^{\prime}\right)\right\rangle \supset\left\langle f_{3} \stackrel{h}{\leftrightarrow} f_{2}, f_{1}\right\rangle+\left\langle f_{3} \stackrel{h}{\leftrightarrow} f_{2}, f_{1}^{\prime}\right\rangle .
$$

The indeterminacy on the left-hand side consists precisely of adding elements of the form $f_{3} u$, while on the right-hand side it consists of adding elements of the form $f_{3} v+f_{3} v^{\prime}=f_{3}\left(v+v^{\prime}\right)$. Because the indeterminacy group is the same, this containment must be an equality of cosets.

Now letting $h$ vary over possible tetherings (which produces a restricted set of elements on the right-hand side), we obtain the third identity.

\subsection{Model categories}

Working in a model category often requires attention to objects that are not cofibrant or fibrant, and function spaces for such objects are poorly behaved. In this section we will spell out adjustments to the construction of secondary operations which are more convenient but equivalent to our standard construction.

Let $\mathcal{M}$ be a model category. Associated to this data there is a hammock localization $L^{H} \mathcal{M}$ [DK80a]. This is a simplicial category with a functor $\mathcal{M} \rightarrow L^{H} \mathcal{M}$, bijective on objects, that turns weak equivalences into homotopy equivalences. In [DK80b] it is shown that $L^{H} \mathcal{M}$ recovers the homotopy theory of $\mathcal{M}$ : it is invariant under Quillen equivalence, the homotopy category of $L^{H} \mathcal{M}$ is localization of $\mathcal{M}$ with respect to weak equivalences, and if $\mathcal{M}$ is a simplicial model category there is a chain of weak equivalences between $L^{H} \mathcal{M}$ and the simplicial category of cofibrant-fibrant objects of $\mathcal{M}$.

With this in mind, for (possibly pointed or augmented) objects of $\mathcal{M}$ it makes sense to calculate secondary composites and brackets in either $\mathcal{M}$ or $L^{H} \mathcal{M}$. There are natural maps

$$
\pi_{k} \operatorname{Map}_{\mathcal{M}}(X, Y) \rightarrow \pi_{k} \operatorname{Map}_{\mathcal{M}}\left(X_{c o f}, Y_{f i b}\right) \cong \pi_{k} \operatorname{Map}_{L^{H}}(X, Y),
$$

where the first is an isomorphism if $X$ is cofibrant and $Y$ is fibrant. This natural map is compatible with function composition.

This means that a tethering, secondary composite, secondary operation, or bracket in $\mathcal{M}$ determines a compatible one in $L^{H} \mathcal{M}$. This use of $L^{H} \mathcal{M}$ then allows us to discuss brackets, and identities between them, for maps in the homotopy category of $\mathcal{M}$ without the inconvenience of using cofibrant or fibrant replacements to obtain maps in $\mathcal{M}$. When discussing secondary composites in $\mathcal{M}$, we will regard this process as implicit. 


\subsection{Secondary power operations}

The study of secondary operations can now be specialized to homotopy operations for algebras over a fixed commutative ring spectrum $A$.

Definition 2.6.1. Given a commutative ring spectrum $A$, we let $\mathbb{P}_{A}^{E_{n}}$ be the left adjoint to the forgetful functor from $E_{n} A$-algebras to spectra; if $n=\infty$ we simply write $\mathbb{P}_{A}$, and if $A=\mathbb{S}$ then we will omit $A$ from the notation.

In particular, there is an isomorphism

$$
\mathbb{P}_{A}^{E_{n}}(X) \cong \bigvee A \wedge\left(E_{n}(k)_{+} \wedge \Lambda_{\Sigma_{k}} X^{\wedge k}\right)
$$

where the spaces $E_{n}(k)$ are the terms in our chosen $E_{n}$-operad, and the set of homotopy classes of maps of $E_{n} A$-algebras $\mathbb{P}_{A}^{E_{n}}\left(\vee S^{k_{i}}\right) \rightarrow C$ is naturally isomorphic to $\prod \pi_{k_{i}} C$. The natural map $X \rightarrow *$ becomes a natural augmentation $\mathbb{P}_{A}^{E_{n}}(X) \rightarrow A$, and a pinch map $X \rightarrow X \vee X$ gives $\mathbb{P}_{A}^{E_{n}}(X)$ the structure of a co-H-object.

Definition 2.6.2. A homotopy operation on $E_{n} A$-algebras is a natural transformation of functors

$$
\prod \pi_{k_{i}}(-) \rightarrow \pi_{j}(-)
$$

represented by a homotopy class of map of $E_{n} A$-algebras

$$
\mathbb{P}_{A}^{E_{n}}\left(S^{j}\right) \rightarrow \mathbb{P}_{A}^{E_{n}}\left(\vee S^{k_{i}}\right)
$$

or equivalently an element of

$$
\pi_{j} \mathbb{P}_{A}^{E_{n}}\left(\vee S^{k_{i}}\right) \cong \pi_{j}\left(A \wedge \mathbb{P}^{E_{n}}\left(\vee S^{k_{k}}\right)\right)
$$

If this operation preserves the zero element, we view it as determined by a map of augmented objects via the canonical projection to $A$; if it preserves addition, we view it as determined by a map of co-H-objects.

Similarly, if $B$ is an $E_{n} A$-algebra, a homotopy operation on $E_{n}$ A-algebras under $B$ is a natural transformation in the homotopy category of $E_{n} A$-algebras under $B$, represented by a homotopy class of map

$$
B \amalg \mathbb{P}_{A}^{E_{n}}\left(S^{j}\right) \rightarrow B \amalg \mathbb{P}_{A}^{E_{n}}\left(\vee S^{k_{i}}\right) .
$$

Here the coproduct $U$ takes place in the category of $E_{n}$-algebras. If this operation preserves the zero element, we view it as determined by a map of augmented objects via the canonical projection to $B$; if it preserves addition, we view it as determined by a map of co-H-objects.

Taking $B=A$ shows that the first type of operations are a special case of the second, so there is no loss of generality in restricting our attention to operations in the relative case. If $n=\infty$, then conversely $E_{\infty} A$-algebras under $B$ are equivalent to $E_{\infty} B$-algebras. 
Example 2.6.3. For any $b \in \pi_{k}(B)$ and any $n>0$, multiplication by $b$ determines an additive homotopy operation on $E_{n} A$-algebras under $B$.

Remark 2.6.4. As above, the Yoneda lemma allows homotopy operations to be expressed as pre-composition with maps of free algebras. We usually write precomposition on the right, but this is at odds with the standard convention of writing operators (such as the Dyer-Lashof operations) on the left. We could attempt to solve this in many ways. One would be to work in an opposite category so that function application is on the right. One would be to notationally distinguish between maps between free algebras (operations), maps from free algebras to ordinary algebras (homotopy elements), and maps between ordinary algebras (maps). One is to accept the state of affairs, and resist the urge to use the same names for a Dyer-Lashof operation $Q^{n}$ and the map $\mathbb{P}_{H}\left(S^{j+n}\right) \rightarrow \mathbb{P}_{H}\left(S^{j}\right)$ that represents it. None of these solutions are good, but we have adopted the third because (in all honesty) it has confused us the least.

Relations between homotopy operations allow us to define secondary operations in the following way.

Definition 2.6.5. Let $A$ be a commutative ring spectrum and $B$ an $E_{n} A$-algebra. Suppose we have homotopy operations $Q_{i}: \prod_{s} \pi_{l_{i, s}} \rightarrow \pi_{k_{i}}$ and $R: \prod_{i} \pi_{k_{i}} \rightarrow \pi_{j}$ that preserve zero such that $R \circ\left(\prod_{i} Q_{i}\right)=0$, realized by a homotopy coherent diagram

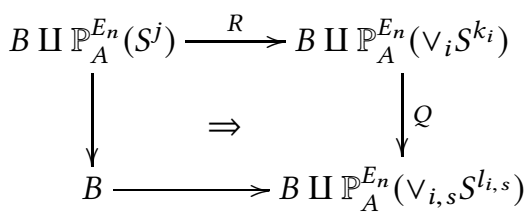

of augmented $E_{n} A$-algebras under $B$. We refer to $R$ as a relation between the operations $Q_{i}$. The coherence produces a tethering homotopy $h$, and the secondary operation associated to this relation is $\langle-, Q \stackrel{h}{\leftrightarrow} R\rangle$.

Proposition 2.6.6. Given $C$ any $E_{n}$ A-algebra under $B$, the domain of definition of the secondary operation $\langle-, Q \stackrel{h}{\leftrightarrow} R\rangle$ is the subset of $\prod \pi_{l_{i, s}} C$ of collections of elements $x_{i, s} \in$ $\pi_{l_{i, s}} C$ such that $Q_{i}\left(x_{i, s}\right)=0$ for all $i$. These are represented by homotopy commutative diagrams

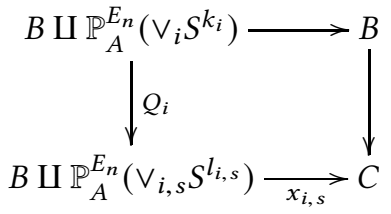

of $E_{n} A$-algebras under $B$. The value of $\langle-, Q \stackrel{h}{\leftrightarrow} R\rangle$ is a subset of $\pi_{j+1} C$, and the indeterminacy consists of adding elements in the image of the suspended operation $\sigma R: \prod \pi_{k_{i}+1} C \rightarrow$ $\pi_{j+1} C$.

Proposition 2.6.7. Maps $f: C \rightarrow D$ of $E_{n}$ A-algebras under B preserve secondary operations. 
Proof. This is the statement that

$$
f(\langle x, Q \leadsto R\rangle) \subset\langle f(x), Q \leadsto R\rangle,
$$

which is an application of the juggling formulas from Proposition 2.3.4.

Remark 2.6.8. If $n<m \leq \infty$, then the forgetful functors from $E_{m} A$-algebras under $B$ to $E_{n} A$-algebras under $B$ also preserve secondary operations in the following sense. The forgetful functor $U$ from $E_{m} A$-algebras under $B$ to $E_{n}$-algebras under $B$ has a left adjoint $F$, giving rise to an enriched adjunction. Since adjoints are preserved under composition, it preserves free objects:

$$
F\left(B \amalg \mathbb{P}_{A}^{E_{n}}(X)\right) \cong B \amalg \mathbb{P}_{A}^{E_{m}}(X) .
$$

In particular, any homotopy operation

$$
Q: B \amalg \mathbb{P}_{A}^{E_{n}}\left(S^{j}\right) \rightarrow B \amalg \mathbb{P}_{A}^{E_{n}}\left(\vee_{i} S^{k_{i}}\right)
$$

for $E_{n} A$-algebras under $B$ gives rise to a homotopy operation for $E_{m} A$-algebras under $B$, defined by applying $F Q$ or, equivalently, by applying $U$ and then applying $Q$. By Corollary 2.1.12, the enriched adjunction gives us canonical identifications

$$
\langle U-, Q \stackrel{h}{\leftrightarrow} R\rangle_{E_{n}}=\langle-, F Q \stackrel{F h}{\leftrightarrow} F R\rangle_{E_{m}}
$$

showing that secondary operations are preserved by the forgetful functor.

We can also define functional homotopy operations as the analogues of Steenrod's functional cohomology operations.

Definition 2.6.9. Suppose $A$ is a commutative ring spectrum and that we have maps $B \rightarrow C \stackrel{f}{\rightarrow} D$ of $E_{n} A$-algebras, making $f: C \rightarrow D$ a map under $B$. Suppose that we have a homotopy operation $Q: \prod_{s} \pi_{l_{s}} \rightarrow \pi_{k}$ for $E_{n} A$-algebras under $B$ that preserves zero, realized by a commutative diagram



The functional homotopy operation associated to this relation is the bracket $\langle f,-, Q\rangle$.

Proposition 2.6.10. For any maps of $E_{n}$ A-algebras $B \rightarrow C \stackrel{f}{\rightarrow} D$, the domain of definition of the functional operation $\langle f,-, Q\rangle$ is the subset of $\prod \pi_{l_{s}} C$ of collections of elements $x_{s} \in \pi_{l_{s}} C$ such that $f\left(x_{s}\right)=0$ and $Q\left(x_{s}\right)=0$. These are represented by homotopy commutative diagrams

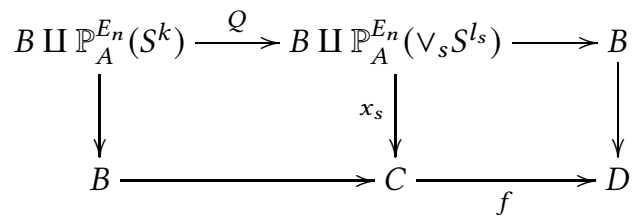


of $E_{n} A$-algebras. The value of $\langle f,-, Q\rangle$ is a subset of $\pi_{k+1} D$, and the indeterminacy consists of adding elements in the image of the suspended operation $\sigma Q: \prod \pi_{l_{s}+1} D \rightarrow$ $\pi_{k+1} D$ and elements in the image of $f: \pi_{k+1} C \rightarrow \pi_{k+1} D$.

We now specialize the previous discussion to the category of $E_{n}$-algebras over the mod-2 Eilenberg-Mac Lane spectrum $H$. As in Example 2.6.3, multiplication is one classical example of a homotopy operation. Other examples of homotopy operations, and relations between them, are furnished by power operations.

Theorem 2.6.11 ([BMMS86, III.3]). For any commutative $H$-algebra A, there are homotopy operations

$$
Q^{s}: \pi_{k} \rightarrow \pi_{k+s}
$$

for $E_{n}$ A-algebras when $s<k+n-1$. These satisfy the following relations.

1. The additivity relation: $Q^{s}(x+y)=Q^{s}(x)+Q^{s}(y)$

2. The instability relations: $Q^{s} x=x^{2}$ when $|x|=s, Q^{s} x=0$ when $|x|>s$

3. The Cartan formula: $Q^{s}(x y)=\sum_{p+q=s} Q^{p}(x) Q^{q}(y)$

4. The Adem relations: If $r>2 s, Q^{r} Q^{s}(x)=\sum\left(\begin{array}{c}i-s-1 \\ 2 i-r\end{array}\right) Q^{r+s-i} Q^{i}$

For $m \leq n$, the forgetful map from $E_{n}$-algebras to $E_{m}$-algebras preserves Dyer-Lashof operations.

Proposition 2.6.12. For any commutative H-algebra A, all homotopy operations for $E_{\infty} A$-algebras $C$ are composites of the following types:

1. the constant operation associated to an element $\alpha \in \pi_{n} A$, which takes no arguments and whose value on $C$ is the image of $\alpha$ under the map $\pi_{*} A \rightarrow \pi_{*} C$;

2. the Dyer-Lashof operations $Q^{s}: \pi_{n}(C) \rightarrow \pi_{n+s}(C)$;

3. the binary addition operations $\pi_{n}(C) \times \pi_{n}(C) \rightarrow \pi_{n}(C)$;

4. the binary multiplication operations $\pi_{n}(C) \times \pi_{m}(C) \rightarrow \pi_{n+m}(C)$.

Proof. The set of homotopy operations $\prod_{s} \pi_{l_{s}} \rightarrow \pi_{*}$ in this category is isomorphic to

$$
\pi_{*}\left(A \wedge \mathbb{P}\left(\vee_{s} S^{l_{s}}\right)\right) \cong \pi_{*} A \otimes H_{*} \mathbb{P}\left(\vee_{s} S^{l_{s}}\right) .
$$

Therefore, any homotopy operation is a sum of homotopy operations for $\mathrm{H}$-algebras multiplied by constants from $\pi_{*} A$. However, in [BMMS86, IX.2.1] it is shown that the homology $H_{*} \mathbb{P}(X)$ is the free commutative algebra with Dyer-Lashof operations (subject to the additivity formula, instability relations, Cartan formula, and Adem relations) on $H_{*} X$, and so the homotopy operations for $H$-algebras are generated by constants, addition, multiplication, and the Dyer-Lashof operations $Q^{s}$.

The category of $E_{n} A$-algbras under $B$ has suspensions, and the suspension of the augmented object $B \amalg \mathbb{P}_{A}^{E_{n}}\left(\vee_{s} S^{l_{s}}\right)$ is $B \amalg \mathbb{P}_{A}^{E_{n}}\left(\vee_{s} S^{l_{s}+1}\right)$ 
Proposition 2.6.13. The suspension operator $\sigma$, on homotopy operations for $E_{n} A$ algebras under $B$, takes zero-preserving homotopy operations $\prod \pi_{l_{s}} \rightarrow \pi_{k}$ to homotopy operations $\prod \pi_{l_{s}+1} \rightarrow \pi_{k+1}$. Suspension preserves addition, composition, and multiplication by scalars from B. Suspension also takes $Q^{s}$ to $Q^{s}$ and takes the binary multiplication operation $\pi_{p} \times \pi_{q} \rightarrow \pi_{p+q}$ to the trivial operation.

Remark 2.6.14. For $E_{n} H$-algebras, there is also a "top" operation $\xi_{n-1}$ which, if $C$ extends to an $E_{n+1}$-algebra, agrees with to $Q^{k+n-1}$ on classes in $\pi_{k} C$. However, the top operation satisfies less tractable versions of the identities enjoyed by the remaining operations-most prominently, additivity requires correction by a new binary operation called the Browder bracket [BMMS86, III.3.3].

\subsection{Spectra and geometric realization}

For the following, we note that a tethering of a composite map of spectra $X \stackrel{f}{\rightarrow} Y \stackrel{g}{\rightarrow} Z$ is equivalent to a homotopy class of extension from the mapping cone $C f$ to $Z$, up to orientation for the interval component of the mapping cone.

Proposition 2.7.1. Suppose $X, Y$, and $Z$ are spectra, $X \stackrel{f}{\rightarrow} Y \stackrel{g}{\rightarrow} Z$ is nullhomotopic, and that $\alpha \in \operatorname{ker}(f) \subset \pi_{n}(X)$ is represented by a map $S^{n} \rightarrow X$. Given any extension $Y \rightarrow C f \stackrel{h}{\rightarrow} Z$ from the mapping cone representing a tethering, the secondary operation $\langle g \leftrightarrow f, \alpha\rangle$ is (up to sign) the set $h\left(\partial^{-1} \alpha\right)$, where $\partial: \pi_{n+1} C f \rightarrow \pi_{n} X$ is the connecting homomorphism in the long exact sequence of homotopy groups.

Corollary 2.7.2. Suppose that $X_{\star}$ is a simplicial spectrum with geometric realization $\left|X_{\star}\right|$ and that $F$ is the homotopy fiber in the sequence $F \stackrel{j}{\rightarrow} X_{1} \stackrel{d_{0}}{\rightarrow} X_{0}$. Then the composite $F \stackrel{d_{1} j}{\longrightarrow} X_{0} \stackrel{i}{\rightarrow}\left|X_{\star}\right|$ has a canonical tethering. If $\alpha \in \pi_{n}(F) \subset \pi_{n} X_{1}$ is in the kernel of $d_{1}$, then in the geometric realization spectral sequence

$$
H_{p}\left(\pi_{q} X_{\star}\right) \Rightarrow \pi_{p+q}\left|X_{\star}\right|
$$

the secondary operation $\left\langle i \rightsquigarrow d_{1} j, \alpha\right\rangle$ is represented (up to sign) by the element $[\alpha] \epsilon$ $H_{1}\left(\pi_{n} X_{\star}\right)$ in the spectral sequence.

Proof. The 1-skeleton of $\left|X_{\star}\right|$, by definition, has a canonical diagram



This defines a homotopy between the maps $i d_{0}$ and $i d_{1}$. The map $d_{0} j$ has a canonical nullhomotopy by definition, and composing these two homotopies gives a canonical tethering

$$
i\left(d_{1} j\right) \Rightarrow i\left(d_{0} j\right) \Rightarrow *
$$


of $i\left(d_{1} j\right)$. In particular, there is a canonical map $C j \rightarrow s k^{(1)}\left|X_{\star}\right|$ from the mapping cone of $j$ to the 1-skeleton of the geometric realization; by more carefully understanding the degeneracies, we can show that this map is a homotopy equivalence.

By Proposition 2.7.3, in the resulting long exact sequence

$$
\ldots \pi_{n+1} X_{0} \stackrel{i}{\rightarrow} \pi_{n+1} s k^{(1)}\left|X_{\star}\right| \stackrel{\partial}{\rightarrow} \pi_{n} F \stackrel{d_{1} j}{\rightarrow} \pi_{n} X_{0} \stackrel{i}{\rightarrow} \ldots
$$

any $\alpha \in \pi_{n}(F)$ which maps to zero under $d_{1} j$ has a bracket $\left\langle i \leftrightarrow d_{1} j, \alpha\right\rangle$ in $\pi_{n+1} s k^{(n+1)}\left|X_{\star}\right|$, represented by any lift of $\alpha \in \pi_{n} F$, with indeterminacy given by the image of $i$.

The spectral sequence for the homotopy groups of the geometric realization $\left|X_{\star}\right|$ is the spectral sequence associated to the following (unrolled) exact couple:

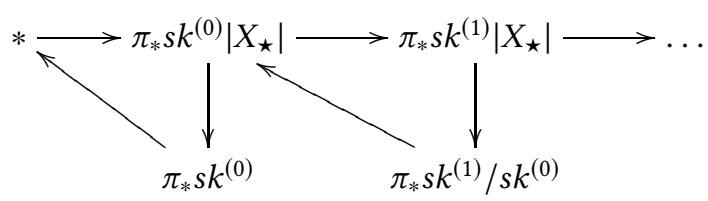

Identifying the 0 -skeleton with $X_{0}$ and the next layer with the suspension of $F$, we obtain our desired identification of the element in the $E_{1}$-term with $\alpha$.

We will now specialize to discuss how certain elements in a Künneth spectral sequence can be identified with the results of secondary operations.

Proposition 2.7.3. Suppose $f: R \rightarrow S$ is a map of commutative ring spectra, and let $i=1 \wedge f: S \wedge R \rightarrow S \wedge S$. Then, in the (pointed) category of augmented commutative $S$-algebras, there is a canonical tethering $p \stackrel{t}{\sim} \rightarrow$ for the composite

$$
S \wedge R \stackrel{i}{\rightarrow} S \wedge S \stackrel{p}{\rightarrow} S \wedge \underset{R}{\wedge}
$$

Let $x \in \pi_{n}(S \wedge R)$ map to zero in $\pi_{n}(S \wedge S)$, so that $\sigma x=\langle p \stackrel{t}{\leftrightarrow} i, x\rangle \subset \pi_{n+1} S \wedge_{R} S$ is defined. Then $\sigma x$ is detected by the image of $x$ under $\pi_{n}(S \wedge R) \rightarrow \pi_{n}(S \wedge R \wedge S)$ in the two-sided bar construction spectral sequence

$$
H_{p} \pi_{q}\left(S \wedge R^{\wedge \star} \wedge S\right) \Rightarrow \pi_{p+q}(S \underset{R}{\wedge} S)
$$

Proof. The relative smash product receives a map from the end of the augmented simplicial bar construction

$$
S \wedge R \wedge S \Longrightarrow S \wedge S \longrightarrow S \wedge_{R} S
$$

a diagram of commutative ring spectra. The face maps

$$
d_{j}: S \wedge R \rightarrow S \wedge R \wedge S \rightarrow S \wedge S
$$

are the null map $S \wedge R \rightarrow S \stackrel{\eta_{L}}{\rightarrow} S \wedge S$ for $j=0$ and the map $S \wedge R \stackrel{i}{\rightarrow} S \wedge S$ for $j=1$. Because the two composites $S \wedge R \rightarrow S \wedge_{R} S$ are homotopic, this provides a canonical tethering in the category of $S$-algebras. 
A homotopy element $x$ as described comes from a homotopy coherent diagram as follows:

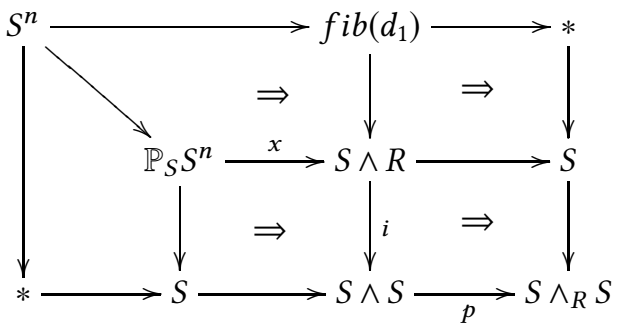

The two lower right-hand squares define the bracket $\langle p, i, x\rangle$ in augmented commutative $S$-algebras, while the outside of the diagram is made up of two large (2-by-2 and 2-by-1) rectangles that are the result of forgetting down to spectra. However, by Corollary 2.7.2 the outside square determines an element of $\pi_{n+1}\left(S \wedge_{R} S\right)$ which lifts to the desired element in the two-sided bar construction spectral sequence.

Remark 2.7.4. The tethering plays an important role here. If we do not impose that the tethering $p \stackrel{t}{\leftrightarrow} i$ comes from a tethering in $E_{\infty}$-algebras, rather than spectra, then the indeterminacy for the bracket in spectra is too large to determine anything about bracket in $E_{\infty}$-algebras.

If $\pi_{*}(S \wedge R)$ is flat over $\pi_{*} S$, we can identify the $E_{2}$-term in the two-sided bar construction spectral sequence:

$$
E_{* *}^{2}=\operatorname{Tor}_{* *}^{\pi_{*}(S \wedge R)}\left(\pi_{*}(S \wedge S), \pi_{*} S\right) \Rightarrow \pi_{*}(S \underset{R}{\wedge} S)
$$

The element $x$ gives rise to the corresponding element in Tor $_{1, n}$. In particular, we have the following result when the target is the mod-2 Eilenberg-Mac Lane spectrum.

Proposition 2.7.5. Suppose $R \rightarrow H$ is a map of $E_{\infty}$-algebras and $x \in H_{n} R$ maps to zero in the dual Steenrod algebra $H_{*} H$. Then there is an element $\sigma x=\langle p \stackrel{t}{\leftrightarrow} i, x\rangle$ in the $R$-dual Steenrod algebra $\pi_{*}\left(H \wedge_{R} H\right)$ which is detected by the image of $x$ in homological filtration 1 of the spectral sequence

$$
\operatorname{Tor}_{* *}^{H_{*} R}\left(H_{*}, H_{*} H\right) \Rightarrow \pi_{*}(H \underset{R}{\wedge} H)
$$

Proof. In this case, we can rectify the map $R \rightarrow H$ to a weakly equivalent map between commutative ring spectra and apply Proposition 2.7.3.

We now specialize this result to the case where $R$ is the complex bordism spectrum.

Proposition 2.7.6. Let $n$ be an integer which is not of the form $2^{k}-1$ for any $k$, so that the corresponding generator $b_{n} \in H_{2 n} M U \cong \mathbb{F}_{2}\left[b_{1}, b_{2}, \ldots\right]$ in mod-2 homology is the Hurewicz image of the generator $x_{n} \in \pi_{2 n} M U \cong \mathbb{Z}\left[x_{1}, x_{2}, \ldots\right]$. Then the diagram of $E_{\infty}$ H-algebras

$$
\mathbb{P}_{H}\left(S^{2 n}\right) \stackrel{b_{n}}{\rightarrow} H \wedge M U \stackrel{p}{\rightarrow} H \wedge H \stackrel{i}{\rightarrow} H \underset{M U}{\wedge} H,
$$

determines a bracket, and $\left\langle p, i, b_{n}\right\rangle \equiv \sigma x_{n} \bmod$ decomposables. 
Proof. The map $H_{*} M U \rightarrow H_{*} H$ is isomorphic to a map of polynomial algebras

$$
\mathbb{F}_{2}\left[b_{1}, b_{2}, \ldots\right] \rightarrow \mathbb{F}_{2}\left[\xi_{1}, \xi_{2}, \ldots\right]
$$

that sends $b_{2^{k}-1}$ to $\xi_{k}^{2}$ and sends the other generators to zero [Rav86, 3.1.4]. In particular, the Künneth spectral sequence

$$
\operatorname{Tor}_{* *}^{H_{*} M U}\left(H_{*}, H_{*} H\right) \Rightarrow \pi_{*}(H \underset{M U}{\wedge} H)
$$

has as $E_{2}$-term an exterior algebra $\Lambda\left[\xi_{k}\right] \otimes \Lambda\left[\sigma b_{n} \mid k \neq 2^{k}-1\right]$. By comparison with the Künneth spectral sequence

$$
\operatorname{Tor}_{* *}^{\pi_{*} M U}\left(H_{*}, H_{*}\right) \Rightarrow \pi_{*}(H \underset{M U}{\wedge} H),
$$

which degenerates and has $E_{2}$-term $\Lambda\left[\sigma x_{k}\right]$ of the same (graded) dimension, we find that spectral sequence (2.1) degenerates and that $\sigma b_{n}$ is congruent to $\sigma x_{n}$ mod decomposables for $n$ not of the form $2^{k}-1$. We can then apply Proposition 2.7.5 to identify $\sigma x_{n}$ as a secondary operation.

\section{Hopf rings}

\subsection{Background}

In this section we will recall some of the work of Ravenel-Wilson on Hopf rings [RW74].

Let $E$ be a spectrum with a homotopy commutative multiplication and let $\left\{E_{n}\right\}_{n \in \mathbb{Z}}$ be an associated $\Omega$-spectrum. Then for any ring $R$ the homology groups $H_{*}\left(E_{\star}, R\right)$ have the structure of a Hopf ring: they have a coproduct $\Delta$, an additive product \#, and a multiplicative product $\circ$ satisfying associativity, commutativity, unitality, and distributivity laws that make them into a graded ring object in coalgebras [RW74, 1.12]. ${ }^{5}$ The constants $c \in E^{n}=\pi_{0} E_{n}$ give rise to elements $[c] \in H_{0}\left(E_{n} ; R\right)$ under the Hurewicz map.

Definition 3.1.1. Suppose $E$ has a complex orientation $x \in \widetilde{E}^{2}\left(\mathbb{C P}^{\infty}\right)$ realized by a based map $b: \mathbb{C P}^{\infty} \rightarrow E_{2}$, and let $\beta_{i} \in H_{2 i}\left(\mathbb{C P}^{\infty} ; R\right)$ be dual to the generator $t^{i} \in$ $H^{*}\left(\mathbb{C P}^{\infty} ; R\right) \cong R[t]$. We define the classes $b_{i} \in H_{2 i}\left(E_{2} ; R\right)$ to be the images of $\beta_{i}$ under $f$.

Theorem 3.1.2 ([RW74, 4.6, 4.15, 4.20]). Let $\left\{M U_{n}\right\}$ be an $\Omega$-spectrum associated to complex cobordism. For any ring $R$ and any $n \in \mathbb{Z}, H_{*}\left(M U_{2 n} ; R\right)$ is, as an algebra under $\#$, the tensor product of the group algebra $\mathbb{Z}\left[\pi_{-2 n} M U\right]$ with a polynomial algebra over $R$.

The even-degree indecomposables $Q^{\#} \widetilde{H}_{*}\left(M U_{2 \star} ; R\right)$ under the \#-product form a commutative graded ring under $\circ$, with relations as follows. If we define a formal power

\footnotetext{
${ }^{5}$ Ravenel-Wilson write $x * y$ for the additive product and $x \circ y$ for the multiplicative product, while Cohen-Lada-May [CLM76] write $x y$ for the additive product and $x \# y$ for the multiplicative product.
} 
series $b(s)=\sum b_{i} s^{i}$ and write $x+{ }_{F} y=\sum a_{i, j} x^{i} y^{j}$ for the formal group law of $M U_{*}$, then we have the Ravenel-Wilson relations

$$
b(s+t)=\sum\left[a_{i, j}\right] \circ b(s)^{\circ i} \circ b(t)^{\circ j} .
$$

The ring $Q^{\#} \widetilde{H}_{*}\left(M U_{2 \star} ; R\right)$ is a quotient of the graded ring

$$
R\left[b_{i}\right] \otimes M U^{-2 \star}
$$

by a regular sequence, determined by the Ravenel-Wilson relations. Both $Q^{\#} \widetilde{H}_{*}\left(M U_{2 \star} ; R\right)$ and $H_{*}\left(M U_{2 \star} ; R\right)$ are free over $R$.

Corollary 3.1.3. For all $n$ and all primes $p$, we have commutative diagrams of the following form:

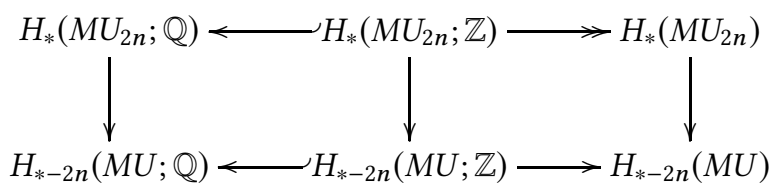

\subsection{The unstable homology invariant}

In the following, for spaces $X$ and $Y$ we will find it convenient to identify $\operatorname{Hom}\left(H_{*} X, H_{*} Y\right)$ with the isomorphic completed tensor product

$$
H_{*}(Y) \hat{\otimes} H^{*}(X) .
$$

Here $H_{*}(Y)$ is discrete, while $H^{*}(X)$ inherits an inverse limit structure dual to the filtration of $H_{*}(X)$ by finite-dimensional subspaces.

The invariant below, in a slightly different form, appears as the "total unstable operation" in [Goe99, 10.2] and is credited to Strickland.

Definition 3.2.1. Let $E$ be a multiplicative generalized cohomology theory represented by an $\Omega$-spectrum $\left\{E_{n}\right\}_{n \in \mathbb{Z}}$. The unstable homology invariant for $E$-cohomology is the collection of natural transformations of sets

$$
\Lambda: E^{n}(X)=\left[X, E_{n}\right] \rightarrow \operatorname{Hom}\left(H_{*} X, H_{*} E_{n}\right) \cong H_{*} E_{n} \hat{\otimes} H^{*}(X) .
$$

Remark 3.2.2. For any $\alpha$, the element $\Lambda(\alpha) \in \operatorname{Hom}\left(H_{*} X, H_{*} E_{n}\right)$ is a coalgebra map that respects the Steenrod operations. This restriction will not be necessary for us to take into account here.

The groups $H_{*} E_{n} \hat{\otimes} H^{*}(X)$ have products \# and $\circ$, each individually induced by the corresponding product in the Hopf ring and the cup product in $H^{*}(X)$. Using these, we can determine how $\Lambda$ interacts with the ring structure in $E$-cohomology.

Proposition 3.2.3. The unstable homology invariant $\Lambda$ satisfies the following formulas:

$$
\begin{aligned}
\Lambda(x+y) & =\Lambda(x) \# \Lambda(y) \\
\Lambda(x y) & =\Lambda(x) \circ \Lambda(y) \\
\Lambda([c]) & =[c] \otimes 1
\end{aligned}
$$


More specifically, for an element $z \in H_{k}(X)$ with coproduct $\Delta z=\sum z^{\prime} \otimes z^{\prime \prime}$, we have the identities

$$
\begin{aligned}
\Lambda(x+y)(z) & =\sum(\Lambda x)\left(z^{\prime}\right) \#(\Lambda y)\left(z^{\prime \prime}\right), \\
\Lambda(x y)(z) & =\sum(\Lambda x)\left(z^{\prime}\right) \circ(\Lambda y)\left(z^{\prime \prime}\right) .
\end{aligned}
$$

For $z \in H_{k}(X)$ with augmentation $\epsilon(z) \in H_{k}(*)$ and $c \in E^{n}$, we have

$$
\Lambda([c])(z)=\epsilon(z)[c] .
$$

Proof. Given elements $x, y \in E^{n}(X)$, represented by maps $X \rightarrow E_{n}$, the sum is represented by the composite

$$
X \stackrel{\Delta}{\longrightarrow} X \times X \stackrel{(x, y)}{\longrightarrow} E_{n} \times E_{n} \stackrel{\#}{\longrightarrow} E_{n} .
$$

Similarly, a product is represented by a composite

$$
X \stackrel{\Delta}{\longrightarrow} X \times X \stackrel{(x, y)}{\longrightarrow} E_{p} \times E_{q} \stackrel{\circ}{\longrightarrow} E_{p+q},
$$

and a constant $c \in E^{n}$ by a composite

$$
X \rightarrow * \stackrel{c}{\rightarrow} E_{n} .
$$

The desired identities follow by applying $H_{*}$.

Remark 3.2.4. In particular, for $X=\mathbb{C P}^{\infty}$ with mod-p graded cohomology ring $\mathbb{F}_{p}[t]$, we can view the unstable homology invariant as a map

$$
E^{n}\left(\mathbb{C P}^{\infty}\right) \rightarrow H_{*}\left(E_{n}\right) \llbracket t \rrbracket
$$

When $E$ is complex oriented, the orientation class $x \in E^{2}\left(\mathbb{C P}^{\infty}\right)$ is taken to the power series

$$
\Lambda(x)=\sum_{i \geq 0} b_{i} t^{i} \in H_{*}\left(E_{2}\right) \llbracket t \rrbracket
$$

(Definition 3.1.1) denoted by $b(t)$ in [RW74]. In these terms, Ravenel-Wilson's identity

$$
b(s+t)=b(s)+{ }_{[F]} b(t)=\#_{i, j}\left[a_{i, j}\right] \circ b(s)^{\circ i} \circ b(t)^{\circ j}
$$

is proved by first applying $\Lambda$ to the identity $m^{*}(t)=\sum a_{i, j} s^{i} t^{j}$ in $E^{2}\left(\mathbb{C P}^{\infty} \times \mathbb{C P}^{\infty}\right)$ and then using naturality of $\Lambda$ to write $\Lambda m^{*}(t)=m^{*} b(t)=b(s+t)$.

While we will not require it, it can be clarifying to examine a "reduced" version of this invariant, especially in cases where $X$ has a basepoint. We begin by observing that $\Lambda(\alpha)-[0]$ takes values in reduced homology for any $\alpha \in E^{*}(X)$. 
Definition 3.2.5. Let $E$ be a multiplicative generalized cohomology theory represented by an $\Omega$-spectrum $\left\{E_{n}\right\}_{n \in \mathbb{Z}}$. The reduced unstable homology invariant for $E$ cohomology is the natural transformation of sets

$$
\lambda: E^{n}(X)=\left[X, E_{n}\right] \rightarrow \operatorname{Hom}\left(H_{*} X, \widetilde{H}_{*} E_{n}\right) \cong \widetilde{H}_{*}\left(E_{n}\right) \hat{\otimes} H^{*}(X)
$$

given by $\lambda(\alpha)=\Lambda(\alpha)-[0]$.

The identities for the operator $\Lambda$ translate into ones for $\lambda$ which are particularly transparent when taken mod decomposables for \#.

Proposition 3.2.6. The reduced unstable homology invariant $\lambda$ satisfies the following formulas:

$$
\begin{aligned}
\lambda(x+y) & =\lambda(x)+\lambda(y)+\lambda(x) \# \lambda(y) \\
\lambda(x y) & =\lambda(x) \circ \lambda(y) \\
\lambda([c]) & =[c]-[0]
\end{aligned}
$$

The composite map

$$
E^{\star}(X) \stackrel{\lambda}{\rightarrow} \widetilde{H}_{*}\left(E_{\star}\right) \hat{\otimes} H^{*}(X) \rightarrow\left(Q^{\#} \widetilde{H}_{*}\left(E_{\star}\right)\right) \hat{\otimes} H^{*}(X)
$$

which reduces mod\#-decomposables is a natural homomorphism of graded $E^{\star}$-algebras.

Finally, we consider the case of reduced cohomology.

Proposition 3.2.7. Suppose $\alpha \in \widetilde{E}^{n}(X)$ corresponds to a based map $X \rightarrow E_{n}$. Then the reduced unstable invariant $\lambda(\alpha)$ naturally takes values in $\widetilde{H}_{*} E_{n} \hat{\otimes} \widetilde{H}^{*}(X)$.

Proof. There is a restriction map $\widetilde{H}_{*} E_{n} \hat{\otimes} H^{*}(X) \rightarrow \widetilde{H}_{*} E_{n} \otimes \mathbb{F}_{p}$ induced by the inclusion of the basepoint $* \rightarrow X$. An element $\alpha \in E^{n}(X)$ which restricts to an element $c \in E^{n}(*)$ at the basepoint is sent to the element $\lambda(\alpha)=\Lambda(\alpha)-[0]$ which restricts to $[c]-[0] \in \widetilde{H}_{*} E_{n}$. If the map is based, then $c=0$ and so $\lambda(\alpha)$ lifts to the tensor with reduced cohomology.

\subsection{Unit groups}

For a ring spectrum $E$, the space $S L_{1}(E) \subset \Omega^{\infty} E$ of strict units is the path component of the multiplicative unit $1 \in \pi_{0}(E)$. This construction is functorial in $E$. If we define $\widetilde{E}_{0} \subset E_{0}$ to be the path component of 0 , then there is a homotopy equivalence $\widetilde{E}_{0} \rightarrow$ $S L_{1}(E)$ given by applying [1]\#(-). In particular, there are canonical isomorphisms $\pi_{k}\left(S L_{1}(E)\right) \cong \pi_{k}(E)$ for $k>0$ and $H_{k}\left(S L_{1}(E)\right) \cong H_{k}\left(\widetilde{E}_{0}\right)$. When $E$ is an $E_{\infty}$-algebra, the space of units inherits a corresponding structure.

Theorem 3.3.1 ([May77, IV.1.8]). For E an $E_{\infty}$-algebra, the space $S L_{1}(E)$ has a natural structure of an infinite loop space such that the map

$$
\Sigma_{+}^{\infty} S L_{1}(E) \rightarrow E
$$

is a natural map of $E_{\infty}$-algebras. 
Proposition 3.3.2. Suppose $E$ is an $E_{\infty}$-algebra, $H R$ is an Eilenberg-Mac Lane spectrum for a commutative ring $R$, and $E \rightarrow H R$ is a map of $E_{\infty}$-algebras. Then there is a natural suspension map

$$
\sigma: S L_{1}(E) \rightarrow \Omega S L_{1}(H R \underset{E}{\wedge} H R)
$$

of infinite loop spaces realizing, for $k>0$, the natural map $\pi_{k} E \rightarrow \operatorname{Tor}_{1, k}^{E_{*}}(R, R)$ in the Künneth spectral sequence

$$
\operatorname{Tor}_{* *}^{E_{*}}(R, R) \Rightarrow \pi_{*}(H R \underset{E}{\wedge} H R)
$$

of [EKMM97, IV.4.1].

Proof. Since $S L_{1}$ only depends on connective covers, without loss of generality we can assume that $E$ is connective. We consider the commutative diagram

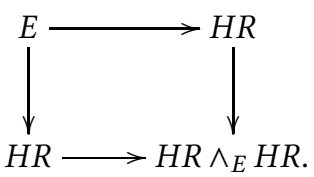

We then apply $S L_{1}$ to this diagram. The space $S L_{1}(H R)$ is contractible, so the commutative diagram of infinite loop spaces

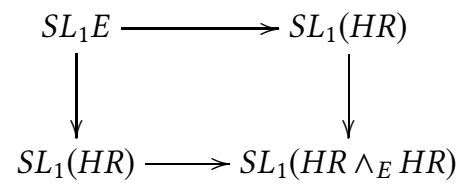

determines (up to contractible indeterminacy) two nullhomotopies of the diagonal map as infinite loop space maps. Gluing these nullhomotopies together gives a map of infinite loop spaces

$$
S L_{1}(E) \rightarrow \Omega S L_{1}(H R \underset{E}{\wedge} H R) .
$$

To show compatibility with the Künneth spectral sequence, we begin by recalling its construction. Setting $H R=M_{0}$, we iteratively find fiber sequences $M_{i+1} \rightarrow F_{i} \rightarrow$ $M_{i}$ of $E$-modules which are exact on homotopy groups, where $F_{i} \simeq \vee_{\alpha} \Sigma^{n_{\alpha}} E$ is a free graded $E$-module, and smash over $E$ with $H R$; the resulting long exact sequences assemble into an exact couple that calculates $\pi_{*}\left(H R \wedge_{E} H R\right)$ with $E_{2}$-term the desired Tor-groups. In particular, we may choose the unit map $E \rightarrow H R$ as one of the factors in the map $F_{0} \rightarrow M_{0}$, which gives us a map $\Sigma_{+}^{\infty} S L_{1}(E) \rightarrow E \rightarrow F_{0}$.

Let $\beta: S^{k} \rightarrow S L_{1}(E)$ represent an element in $\pi_{k} S L_{1}(E)$ for $k>0$, and consider the 
diagram

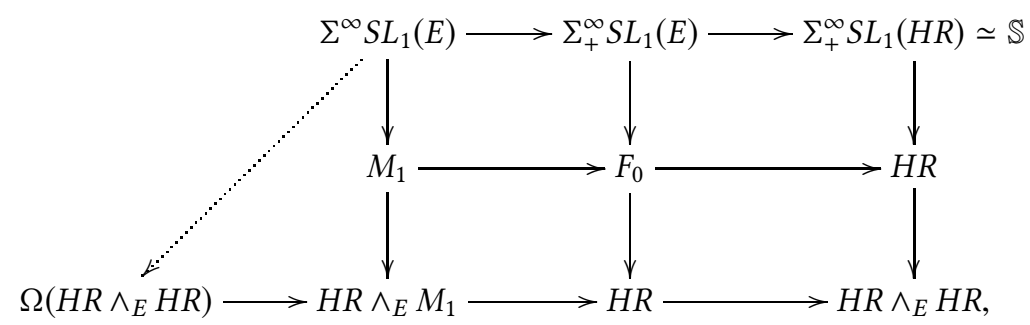

whose rows are fiber sequences and where the dotted arrow is the map induced by the map $\sigma$. The composite map $S^{k} \rightarrow \Sigma^{\infty} S L_{1}(E) \rightarrow M_{1}$ represents the element $\beta \in \operatorname{ker}\left(\pi_{k} E \rightarrow \pi_{k} H R\right)$, and lifts to a map $S^{k} \rightarrow F_{1}$. The image in $\pi_{k}\left(H R \wedge_{E} F_{1}\right)$ is the element corresponding to $\beta$ in $\operatorname{Tor}_{1, k}^{E_{*}}(R, R)$. However, this also coincides with the suspension of $\beta$ under the dotted arrow that uses the two nullhomotopies of $\Sigma^{\infty} S L_{1}(E) \rightarrow H R$.

Corollary 3.3.3. For a ring $R$, there are suspension maps

$$
\sigma: \widetilde{H}_{*}\left(S L_{1}(E) ; R\right) \rightarrow H_{*+1}\left(B S L_{1} E ; R\right) \rightarrow \pi_{*+1}(H R \underset{E}{\wedge} H R) .
$$

These are natural in maps $E \rightarrow H R$ of $E_{\infty}$-algebras, and on the Hurewicz image of $\pi_{*} B S L_{1}(E)$ these are given by the suspension map. When $R=\mathbb{F}_{2}$, this map commutes with the Dyer-Lashof operations.

Proof. The map $S L_{1}(E) \rightarrow \Omega S L_{1}\left(H R \wedge_{E} H R\right)$ is adjoint to a map $B S L_{1}(E) \rightarrow S L_{1}\left(H R \wedge_{E} H R\right)$ of infinite loop spaces. We begin with the map of $E_{\infty}$-algebras

$$
\Sigma_{+}^{\infty} B S L_{1}(E) \rightarrow \Sigma_{+}^{\infty} S L_{1}(H R \underset{E}{\wedge} H R) \rightarrow H R \underset{E}{\wedge} H R .
$$

The adjunction between $E_{\infty}$-algebras and $E_{\infty} H R$-algebras (using the left unit $H R \rightarrow$ $H R \wedge_{E} H R$ ) then produces a natural map

$$
H R \wedge B S L_{1}(E)_{+} \rightarrow H R \wedge \underset{E}{\wedge} H R
$$

of $E_{\infty} H R$-algebras realizing our desired map. In particular, if $R=\mathbb{F}_{2}$ this map of $H$-algebras commutes with the Dyer-Lashof operations.

Corollary 3.3.4. In the commutative diagram

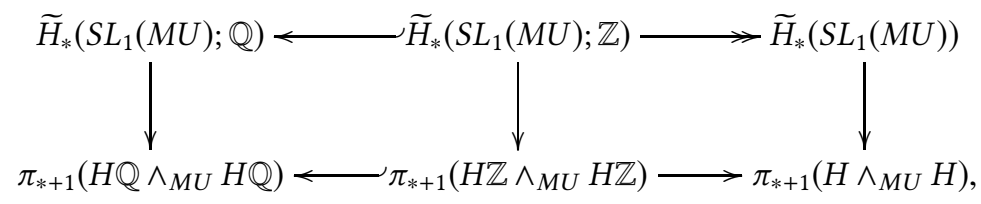

where the vertical maps are suspensions, the left-hand horizontal arrows are injective and the right-hand top horizontal arrow is surjective. In particular, the suspension map in mod-2 homology is determined by the rational suspension map. In addition, the righthand vertical map preserves the Dyer-Lashof operations. 
Proof. The injectivity and surjectivity of the top rows was shown in Corollary 3.1.3. The injectivity of the bottom-left map follows because the comparison map of Künneth spectral sequences

$$
\operatorname{Tor}_{* *}^{\pi_{*} M U}(\mathbb{Z}, \mathbb{Z}) \rightarrow \operatorname{Tor}_{* *}^{\pi_{*} M U}(\mathbb{Q}, \mathbb{Q})
$$

becomes an inclusion of exterior algebras $\Lambda\left[\sigma x_{i}\right] \rightarrow \mathbb{Q} \otimes \Lambda\left[\sigma x_{i}\right]$, and both spectral sequences degenerate at the $E_{2}$-term. Therefore, the map $\pi_{*}\left(H \mathbb{Z} \wedge_{M U} H \mathbb{Z}\right) \rightarrow \pi_{*}\left(H \mathbb{Q} \wedge_{M U} H \mathbb{Q}\right)$ is injective.

We can now examine the properties of the rational suspension map by using the rational Hopf ring.

Proposition 3.3.5. In the rational Hopf ring, the suspension map

$$
\widetilde{H}_{*}\left(S L_{1}(M U) ; \mathbb{Q}\right) \rightarrow H_{*+1}(H \mathbb{Q} \underset{M U}{\wedge} H \mathbb{Q}),
$$

in terms of the Ravenel-Wilson basis, is a composite

$$
\widetilde{H}_{*}\left(S L_{1}(M U) ; \mathbb{Q}\right) \rightarrow Q^{\circ} Q^{\#} \widetilde{H}_{*}\left(M U_{0} ; \mathbb{Q}\right) /\left(b_{2}, b_{3}, \ldots\right) \rightarrow \pi_{*+1}(H \mathbb{Q} \underset{M U}{\wedge} H \mathbb{Q})
$$

that kills \#-decomposables, o-decomposables, and $b_{i}$ for $i>1$, and sends any of the remaining basis elements $[\alpha] \circ b_{1}^{\circ s}$ to the suspension class $\sigma \alpha$ in the Künneth spectral sequence from Proposition 2.7.5.

Proof. There is a commutative diagram of $E_{\infty}$ rings over $H \mathbb{Q}$ :

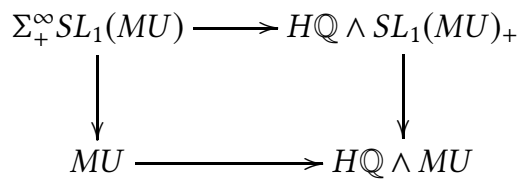

Applying the natural map $\widetilde{H}_{*}\left(S L_{1}(-) ; \mathbb{Q}\right) \rightarrow H \mathbb{Q} \wedge_{(-)} H \mathbb{Q}$, we find that the suspension map

$$
\widetilde{H}_{*}\left(S L_{1}(M U) ; \mathbb{Q}\right) \rightarrow \pi_{*+1}(H \mathbb{Q} \underset{M U}{\wedge} H \mathbb{Q}) \cong \pi_{*+1}(H \mathbb{Q} \underset{H \mathbb{Q} \wedge M U}{\wedge} H \mathbb{Q})
$$

can be computed as the composite

$$
\widetilde{H}_{*}\left(S L_{1}(M U) ; \mathbb{Q}\right) \rightarrow H_{*}(M U ; \mathbb{Q}) \rightarrow H_{*+1}(H \mathbb{Q} \underset{M U}{\wedge} H \mathbb{Q}) .
$$

The first map, under the isomorphism $[-1] \#(-): \widetilde{H}_{*}\left(S L_{1}(M U)\right) \cong \widetilde{H}_{*}\left(\widetilde{M U}_{0}\right)$, sends \#-decomposables to zero, carries o-products to products, and takes the elements $b_{i}$ for $i>1$ to o-decomposable elements $b_{i} \equiv\left[a_{i}\right] \circ b_{1}^{\circ i}$ due to the Ravenel-Wilson relation (3.1). The second is the suspension map $\sigma$, which carries o-decomposables to zero. The element $[\alpha] \circ b_{1}^{\circ s}$ is the Hurewicz image of $\alpha$ which, by definition, is carried to the suspension $\sigma \alpha$.

Taking this together with Corollary 3.3.4, we find the following. 
Corollary 3.3.6. The suspension map

$$
\widetilde{H}_{*}\left(S L_{1}(M U)\right) \rightarrow \pi_{*+1}(H \underset{M U}{\wedge} H)
$$

on mod-2 homology, in terms of the Ravenel-Wilson basis, is a composite

$$
\widetilde{H}_{*}\left(S L_{1}(M U)\right) \rightarrow Q^{\circ} Q^{\#} H_{*}\left(M U_{0}\right) /\left(b_{2}, b_{3}, \ldots\right) \rightarrow \pi_{*+1}(H \underset{M U}{\wedge} H)
$$

that kills \#-decomposables, o-decomposables, and $b_{i}$ for $i>1$, and sends any of the remaining elements $[\alpha] \circ b_{1}^{\circ s}$ in the Ravenel-Wilson basis to the suspension class $\sigma \alpha$ from the Künneth spectral sequence.

Proposition 3.3.7. The suspension map $\sigma$ on mod-2 homology commutes with DyerLashof operations.

Proof. This map is the composite

$$
\widetilde{H}_{*}\left(S L_{1}(M U)\right) \rightarrow H_{*+1}\left(B S L_{1}(M U)\right) \rightarrow \pi_{*+1}(H \underset{M U}{\wedge} H) .
$$

The Dyer-Lashof operations on the homology of infinite loop spaces are stable, and hence preserved by the first map; the compatibility of the second map is Corollary 3.3.3.

\section{Power operations}

\subsection{Power operations in complex oriented theories}

In this section we will recall the work from [BMMS86] on power operations in cohomology theories, and specifically results on $H_{\infty}^{2}$-algebra structures from [BMMS86, VIII].

For an $E_{\infty}$ (and hence $H_{\infty}$ ) ring spectrum $E$, the $E$-cohomology of a (based) space $X$ has natural power operations as follows. Fix $m>0$ and write $D_{m}$ for the extended power functor given by

$$
D_{m}(Y)=\left(Y^{\wedge m}\right)_{h \Sigma_{m}}
$$

Representing an element $\alpha \in E^{0}(X)$ as a map $\alpha: \Sigma^{\infty} X \rightarrow E$, we form the commutative diagram

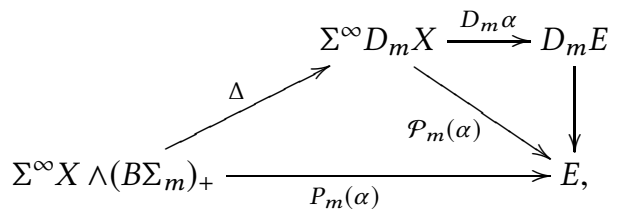

where the right-hand map is induced by the multiplicative structure on $E$. In particular, this produces natural power operations:

$$
\begin{aligned}
\mathcal{P}_{m}: \widetilde{E}^{0}(X) & \rightarrow \widetilde{E}^{0}\left(D_{m} X\right) \\
P_{m}: \widetilde{E}^{0}(X) & \rightarrow \widetilde{E}^{0}\left(X \wedge\left(B \Sigma_{m}\right)_{+}\right)
\end{aligned}
$$


These are multiplicative in an appropriate sense, and by replacing $X$ with $X_{+}$we obtain compatible unbased versions:

$$
\begin{gathered}
\mathcal{P}_{m}: E^{0}(X) \rightarrow E^{0}\left(\left(X^{m}\right)_{h \Sigma_{m}}\right) \\
P_{m}: E^{0}(X) \rightarrow E^{0}\left(X \times B \Sigma_{m}\right)
\end{gathered}
$$

Outside degree 0 , we cannot draw conclusions which are as strong in general. Given an element $\alpha \in \widetilde{E}^{n}(X)$ represented by a map $\Sigma^{\infty} X \rightarrow E \wedge S^{n}$, we can only define part of the desired diagram:

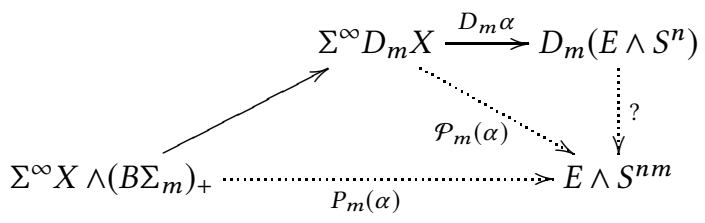

With extra structure on $E$ we can complete this diagram when $n$ is a multiple of some fixed constant $d$ : this is the case where $E$ is $H_{\infty}^{d}$-algebra [BMMS86, I.4]. An $H_{\infty}^{d}$-algebra is an algebra equipped with explicit extra structure maps $D_{m}\left(E \wedge S^{d n}\right) \rightarrow E \wedge S^{d m n}$, multiplicative and compatible across $n$ and $m$. These allow us to obtain power operations:

$$
\begin{aligned}
& \mathcal{P}_{m}: \widetilde{E}^{d k}(X) \rightarrow \widetilde{E}^{d m k}\left(D_{m} X\right) \\
& P_{m}: \widetilde{E}^{d k}(X) \rightarrow \widetilde{E}^{d m k}\left(X \wedge\left(B \Sigma_{m}\right)_{+}\right)
\end{aligned}
$$

These are multiplicative, and replacing $X$ with $X_{+}$gives compatible unbased versions:

$$
\begin{aligned}
& \mathcal{P}_{m}: E^{d k}(X) \rightarrow E^{d m k}\left(\left(X^{m}\right)_{h \Sigma_{m}}\right) \\
& P_{m}: E^{d k}(X) \rightarrow E^{d m k}\left(X \times B \Sigma_{m}\right) .
\end{aligned}
$$

Cohomology is representable, so we may apply the Yoneda lemma. Restricting to the case where $m$ is a chosen prime $p$ and $d=2$, we get the following.

Theorem 4.1.1. If $E$ is an $H_{\infty}^{2}$-algebra, there are natural based and unbased power operations for $n \in \mathbb{Z}$ :

$$
\begin{aligned}
& P: \widetilde{E}^{2 n}(X) \rightarrow \widetilde{E}^{2 p n}\left(X \wedge\left(B \Sigma_{p}\right)_{+}\right) \\
& P: E^{2 n}(X) \rightarrow E^{2 p n}\left(X \times B \Sigma_{p}\right)
\end{aligned}
$$

These are universally represented by maps of based spaces $E_{2 n} \wedge\left(B \Sigma_{p}\right)_{+} \rightarrow E_{2 p n}$, and satisfy $P(x) P(y)=P(x y)$.

For instance, the complex bordism spectrum $M U$ is an $H_{\infty}^{2}$-algebra [BMMS86, VIII.5.1], giving us power operations on even-degree classes previously studied by tom Dieck and Quillen [tD68, Qui71] that extend the power operations in degree zero. The spectrum $M U$, which is complex oriented and has canonical Thom classes for complex vector bundles, also has the special property that these operations are compatible with the Thom isomorphism, as described by Quillen. 
Proposition 4.1.2 ([Qui71]). For any complex vector bundle $\xi \rightarrow X$ of dimension $k$, write $t(\xi) \in M U^{2 k}(T h(\xi))$ for the canonical Thom class of $\xi$ and $e(\xi) \in M U^{2 k}\left(X_{+}\right)$for the Euler class.

The based operation $\mathcal{P}_{m}$ preserves Thom classes: we have

$$
\mathcal{P}_{m}(t(\xi))=t\left(D_{m} \xi\right)
$$

where $D_{m} \xi$ is the extended power bundle over $\left(X^{m}\right)_{h \Sigma_{m}}$. Restricting along the diagonal, we have

$$
P_{m} t(\xi)=e(\xi \otimes \bar{\rho}) t(\xi)
$$

where $\bar{\rho}$ is the bundle on $B \Sigma_{m}$ induced by the reduced permutation representation of $\Sigma_{m}$ and $\xi \otimes \bar{\rho}$ is the exterior tensor bundle on $X \times B \Sigma_{m}$. In particular, the Thom isomorphism fits into a commutative diagram

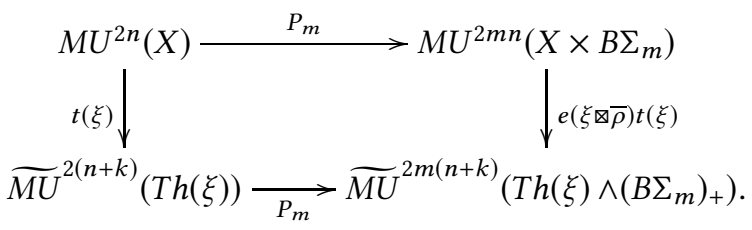

The cohomology of symmetric groups is closely related to formal group law theory [Qui71], and in particular the effect of the power operation $P$ on the canonical first Chern class $x \in \widetilde{M U}^{2}\left(\mathbb{C P}^{\infty}\right)$ was determined by Ando [And95].

Theorem 4.1.3. The inclusion $C_{p} \hookrightarrow \Sigma_{p}$ induces inclusions:

$$
\begin{aligned}
M U^{*}\left(B \Sigma_{p}\right) & \hookrightarrow M U^{*} \llbracket \alpha \rrbracket /[p]_{F}(\alpha) \\
M U^{*}\left(\mathbb{C} \mathbb{P}^{\infty} \times B \Sigma_{p}\right) & \hookrightarrow M U^{*} \llbracket x, \alpha \rrbracket /[p]_{F}(\alpha)
\end{aligned}
$$

In these coordinates, the power operation $P$ satisfies $P(x)=\prod_{i=0}^{p-1}\left(x+{ }_{F}[i]_{F}(\alpha)\right)$.

The power operations $P: M U^{2 k} \rightarrow M U^{2 p k}\left(B \Sigma_{p}\right)$ are in principle determined by these results, naturality, and multiplicativity, and are closely related to the Lubin isogeny in the theory of formal group laws. However, it has been difficult to obtain closed-form expressions for these power operations. We will require the following computation of Johnson-Noel, using the fact that the generator $x_{2}$ of the complex cobordism ring in dimension 4 is $\mathbb{C P}^{2}$.

Theorem 4.1.4 ([JN10, 6.3]). The polynomial generator $x_{2}$ of the complex bordism ring $M U_{*} \cong \mathbb{Z}\left[x_{1}, x_{2}, x_{3}, \ldots\right]$, appearing in $\pi_{4}(M U)$, has image

$$
P\left(x_{2}\right) \equiv \alpha^{2}\left(v_{1}^{6}+v_{2}^{2}\right)+\alpha^{3}\left(v_{1}^{7}+v_{3}\right)
$$

in $B P_{*} \llbracket \alpha \rrbracket /\left([2]_{F}(\alpha), \alpha^{8}\right)$, where $P$ is the 2 -primary power operation. In particular, $P\left(x_{2}\right) \equiv$ $x_{7} \alpha^{3}$ mod decomposables and higher order terms in $\alpha$.

Remark 4.1.5. The powers of $\alpha$ appearing in the above result differ by a shift from those in [JN10] because their identity occurs after multiplication by a power of an Euler class. 
The main result of this paper hinges on this theorem. In Appendix A we will show that Johnson-Noel's method can be adapted to one that works in torsion-free quotients of the Lazard ring. This tweak allows us to give an abbreviated version of their proof at the prime 2, ignoring decomposables, that is easier to carry out without computer assistance.

\subsection{Unstable Dyer-Lashof operations}

We recall the computation of the cohomology of the symmetric group $\Sigma_{p}$ :

$$
H^{*}\left(B \Sigma_{p}\right) \cong \begin{cases}\mathbb{F}_{2}[u] & \text { if } p=2 \\ \mathbb{F}_{p}[u] \otimes \Lambda[v] & \text { if } p>2\end{cases}
$$

Here $u$ has degree 1 if $p=2$, while $u$ has degree $2 p-2$ and $v$ has degree $2 p-3$ if $p$ is odd.

Definition 4.2.1. If $E$ is an $H_{\infty}^{2}$-algebra, the homology power operation

$$
Q: H_{*}\left(E_{2 n}\right) \rightarrow H_{*}\left(E_{2 p n}\right) \hat{\otimes} H^{*}\left(B \Sigma_{p}\right)
$$

is adjoint to the map

$$
H_{*} P: H_{*}\left(E_{2 n}\right) \otimes H_{*}\left(B \Sigma_{p}\right) \rightarrow H_{*}\left(E_{2 p n}\right)
$$

induced by the map $E_{2 n} \wedge\left(B \Sigma_{p}\right)_{+} \rightarrow E_{2 p n}$ of based spaces from Theorem 4.1.1.

The multiplicativity of the natural power operation $P$ has the following consequence.

Proposition 4.2.2. The operation $Q$ satisfies $Q(x) \circ Q(y)=Q(x \circ y)$ and $Q([0])=[0]$.

Proposition 4.2.3. Suppose $E$ is an $H_{\infty}^{2}$-algebra. Then for all $n \in \mathbb{Z}$ we have a commutative diagram of sets



that is natural in $X$. The horizontal maps preserve products and the bottom map is a map of abelian groups.

Proof. The power operation $P$ sends an element represented by a map $\alpha: X \rightarrow E_{2 n}$ to the composite

$$
P(\alpha): X \wedge\left(B \Sigma_{p}\right)_{+} \stackrel{\alpha \wedge 1}{\longrightarrow} E_{2 n} \wedge\left(B \Sigma_{p}\right)_{+} \stackrel{P}{\rightarrow} E_{2 p n} .
$$

The value of $\Lambda(P(\alpha))$ is the effect on homology, which is the composite

$$
H_{*}(X) \otimes H_{*}\left(B \Sigma_{p}\right) \stackrel{H_{*} \alpha \otimes 1}{\longrightarrow} H_{*}\left(E_{2 n}\right) \otimes H_{*}\left(B \Sigma_{p}\right) \stackrel{H_{*} P}{\longrightarrow} H_{*}\left(E_{2 p n}\right) .
$$

Taking adjoints recovers the statement about completed tensor products. 
Remark 4.2.4. The map $B C_{p} \rightarrow \mathbb{C P}^{\infty}$ induces a map $\widetilde{E}^{2}\left(\mathbb{C P}^{\infty}\right) \rightarrow \widetilde{E}^{2}\left(B C_{p}\right)$ that takes the orientation class $x$ to the generator $\alpha$ described in Theorem 4.1.3, and the map $H^{*}\left(\mathbb{C P}^{\infty}\right) \rightarrow H^{*}\left(B C_{p}\right)$ is the ring map that sends $t$ to $u^{2}$ if $p$ is 2 or to a generator $w=u^{1 /(p-1)}$ in degree 2 if $p$ is odd. By naturality of $\Lambda$, we find that $\Lambda(\alpha)$ is equal to $b\left(u^{2}\right)$ if $p=2$ and is equal to $b(w)$ if $p$ is odd.

For the remainder of this section we will focus on the prime 2 . We first recall the following calculation, which is dual to the identity used to define the Steenrod operations in [Ste62, VII.3.2, VII.6.1].

Lemma 4.2.5. For a space $X$ with second extended power $D_{2}(X)$, the composite diagonal map

$$
H_{*}(X) \otimes H_{*}\left(B \Sigma_{2}\right) \rightarrow H_{*}\left(X \times B \Sigma_{2}\right) \rightarrow H_{*}\left(D_{2}(X)\right)
$$

on mod-2 homology is given by

$$
v \otimes \beta_{n} \mapsto \sum_{j \geq 0} Q^{j+n}\left(P_{j} v\right)
$$

Here $\beta_{n}$ is dual to $u^{n}$ and $P_{j}$ is the homology operation dual to $S q^{j}$.

As a result, the Dyer-Lashof operations can be recovered from this diagonal map into the extended power.

Theorem 4.2.6. Consider the homology operations

$$
Q: H_{*}\left(M U_{2 n}\right) \rightarrow H_{*}\left(M U_{4 n}\right) \hat{\otimes} H^{*}\left(B \Sigma_{2}\right)
$$

from Definition 4.2.1. Then there are multiplicative Dyer-Lashof operations

$$
\widehat{Q}^{s}: H_{*}\left(M U_{2 n}\right) \rightarrow H_{*}\left(M U_{4 n}\right)
$$

extending the Dyer-Lashof operations in degree zero of [CLM76, II.1] (coming from the multiplicative $E_{\infty}$-space structure) to Dyer-Lashof operations in even degrees. These satisfy the Cartan formula

$$
\widehat{Q}^{s}(x \circ y)=\sum_{p+q=s} \widehat{Q}^{p}(x) \circ \widehat{Q}^{q}(y)
$$

and are related to $Q$ by the identity

$$
Q(x)=\sum_{n, j} \widehat{Q}^{j+n}\left(P_{j} x\right) u^{n} .
$$

In particular, if all nontrivial Steenrod operations vanish on $x$ then $Q(x)=\sum \widehat{Q}^{n}(x) u^{n}$. This property is invariant under the product 0 . 


\subsection{Power operations in the Hopf ring}

We can now begin to use the results of the previous sections to calculate multiplicative Dyer-Lashof operations in the Hopf ring for $M U$ (the additive ones having been determined by Turner [Tur93]). First we will find the effect on the class $b_{1} \in H_{2}\left(M U_{2}\right)$ of Definition 3.1.1, because o-multiplication by $b_{1}$ represents suspension.

Proposition 4.3.1 (cf. [Pri75]). Let $b_{k} \in H_{2 k}\left(M U_{2}\right)$ denote the fundamental classes of Definition 3.1.1. Then the 2-primary multiplicative Dyer-Lashof operations satisfy

$$
\widehat{Q}^{2 n} b_{1}=b_{1} \circ b_{n}
$$

for all $n \geq 1$.

Proof. For a general prime $p$, we consider the commutative diagram



where the top square expressing compatibility of $P$ with the Thom isomorphism is from Proposition 4.1.2. Because $x$ is the Thom class of the canonical bundle on $B U(1)$, $\Lambda\left(t\left(\gamma_{1}\right)\right)=b(s)$. The image of the unit $1 \in M U^{0}(B U(1))$ along the left-to-bottom composite is then

$$
(Q \otimes 1)(\Lambda(x))=(Q \otimes 1)(b(s))=\sum Q\left(b_{k}\right) s^{k}
$$

On the other hand, the image along the top-right composite is

$$
\Lambda\left(x \prod_{k=1}^{p-1}\left(x+_{F}[k]_{F} \alpha\right)\right)=b(s) \circ\left(b(s)+_{[F]} b\left(u^{2}\right)\right) \circ \cdots \circ\left(b(s)+_{[F]}[p-1]_{[F]} b\left(u^{2}\right)\right),
$$

using the expression for the Euler class of the exterior tensor bundle $\gamma_{1} \otimes \bar{\rho}$ on $B U(1) \times$ $B \Sigma_{p}$

Taking the coefficient of $s$, which involves only the linear term of $b(s)$ and the constant coefficients (in terms of $s$ ) of the factors $b(s){ }_{[F]}[k]_{[F]} b\left(u^{2}\right)$, we find that

$$
Q\left(b_{1}\right)=b_{1} \circ b\left(u^{2}\right) \circ[2]_{[F]} b\left(u^{2}\right) \circ \cdots \circ[p-1]_{[F]} b\left(u^{2}\right) .
$$

When we specialize to $p=2$ and apply Theorem 4.2 .6 , we find

$$
\sum_{j \geq 2} \widehat{Q}^{j}\left(b_{1}\right) u^{j}=b_{1} \circ b\left(u^{2}\right)=\sum_{n \geq 1}\left(b_{1} \circ b_{n}\right) u^{2 n}
$$

as desired. 
Proposition 4.3.2. Suppose that $y \in \pi_{2 n} M U$ and that, in the coordinates of Theorem 4.1.3, we have

$$
P(y)=\sum_{i=0}^{\infty} c_{i} \alpha^{i}
$$

for some elements $c_{i} \in \pi_{4 n+2 i} M U$. Then

$$
Q([y])=\#_{i=0}^{\infty}\left[c_{i}\right] \circ b\left(u^{2}\right)^{\circ i}
$$

Proof. Taking $X=*$ in Proposition 4.2.3 identifying $[y]$ with $\Lambda(y)$, we find

$$
\begin{aligned}
Q([y]) & =\Lambda(P(y)) \\
& =\Lambda\left(\sum c_{i} \alpha^{i}\right) \\
& =\#_{i=0}^{\infty}\left[c_{i}\right] \circ b\left(u^{2}\right)^{\circ i}
\end{aligned}
$$

by Proposition 3.2.3 and Remark 4.2.4.

Corollary 4.3.3. Mod \#-decomposables and the o-ideal generated by $b_{2}, b_{3}, \ldots$, the Hurewicz image $[x] \circ b_{1}^{\circ n} \in H_{2 n}\left(M U_{0}\right)$ of $x \in \pi_{2 n}\left(M U_{0}\right)$ satisfies

$$
Q\left([x] \circ b_{1}^{\circ n}\right) \equiv \sum_{i=0}^{\infty}\left[c_{i}\right] \circ\left(b_{1}\right)^{\circ(i+2 n)} u^{2(i+n)} .
$$

In particular, $\widehat{Q}^{2 k}\left([x] \circ b_{1}^{\circ n}\right) \equiv\left[c_{k-n}\right] \circ b_{1}^{\circ(k+n)}$ in this quotient.

Proof. The first part follows from the multiplication formula $Q\left([x] \circ b_{1}^{\circ n}\right)=Q([x]) \circ$ $Q\left(b_{1}\right)^{\circ n}$. The second part follows from Theorem 4.2.6 and the fact that the operations $P_{j}$ vanish on $[x] \circ\left(b_{1}\right)^{\circ n}$ for $j>0$.

\subsection{Power operations in the $M U$-dual Steenrod algebra}

We will now apply the previous technology to compute a multiplicative Dyer-Lashof operation in $H_{*} S L_{1}(M U)$. In order to do so, we need some preliminary results about how the additive product interacts with multiplicative Dyer-Lashof operations.

Proposition 4.4.1. At $p=2$, the multiplicative and additive Dyer-Lashof operations in the Hopf ring of an $E_{\infty}$-algebra satisfy the following identities.

1. When $x$ and $y$ are in the positive-degree homology of the path component of zero, we have

$$
\widehat{Q}^{s}(x \# y) \equiv Q^{s}(x \circ y)
$$

mod\#-decomposables.

2. When $y$ is in the positive-degree homology of the path component of zero, we have

$$
\widehat{Q}^{s}([1] \# y) \equiv[1] \# Q^{s}(y)+[1] \# \widehat{Q}^{s}(y)
$$

mod\#-decomposables. 
3. For any positive-degree element $x$ there exist elements $z_{i}$ for $0<i<|x|$ such that

$$
Q^{s}(x)=Q^{s}[1] \circ x+\sum Q^{s+i}[1] \circ z_{i}
$$

In particular, $Q^{s}(x)$ is o-decomposable for any $x$ and any $s>0$.

Proof. The mixed Cartan formula [CLM76, II.2.5] takes the following form. If $x$ and $y$ are elements with coproducts given by $\Delta x=\sum x^{\prime} \otimes x^{\prime \prime}$ and $\Delta y=\sum y^{\prime} \otimes y^{\prime \prime}$, then

$$
\widehat{Q}^{s}(x \# y)=\sum_{p+q+r=s} \sum \widehat{Q}^{p}\left(x^{\prime}\right) \# Q^{q}\left(x^{\prime \prime} \circ y^{\prime}\right) \# \widehat{Q}^{r}\left(y^{\prime \prime}\right) .
$$

In the case of the first identity, the only time this is not decomposable under \# is when both $\widehat{Q}^{p}\left(x^{\prime}\right)$ and $\widehat{Q}^{r}\left(y^{\prime \prime}\right)$ are of degree zero; this occurs when $p=r=0$ and we take the terms $[0] \otimes x$ and $y \otimes[0]$ of the coproduct.

In the case of the second identity, the only nonzero terms in the mixed Cartan formula occur when $p=0$ and either $y^{\prime}=[0]$ or $y^{\prime \prime}=[0]$.

The third identity is proven by induction on the degree of $x$, using the formula

$$
Q^{s}([1]) \circ x=\sum Q^{s+i}\left([1] \circ P_{i} x\right)
$$

from [CLM76, II.1.6].

Corollary 4.4.2. When $x$ is in the positive-degree homology of the path component of zero, we have

$$
\widehat{Q}^{s}([1] \# x) \equiv \widehat{Q}^{s}(x)
$$

mod\#-decomposables and o-decomposables, and hence

$$
Q([1] \# x) \equiv Q(x) .
$$

Proof. We have

$$
\begin{aligned}
\widehat{Q}^{s}([1] \# x)-\widehat{Q}^{s}(x) & \equiv[1] \# Q^{s}(x)+[1] \# \widehat{Q}^{s}(x)-[0] \# \widehat{Q}^{s}(x) \\
& =([1]-[0]) \#\left(Q^{s}(x)+\widehat{Q}^{s}(x)\right)+Q^{s}(x) \\
& \equiv 0
\end{aligned}
$$

because the first element is \#-decomposable and the second is o-decomposable.

Proposition 4.4.3. Suppose that $x \in \pi_{2 n} M U$ and that, in the coordinates of Theorem 4.1.3, we have

$$
P(x)=\sum_{i=0}^{\infty} c_{i} \alpha^{i}
$$

for some elements $c_{i} \in \pi_{4 n+2 i} M U$. Then mod\#-decomposables, o-decomposables, and the ideal generated by $b_{2}, b_{3}, \ldots$, the Hurewicz image $[1] \#\left([x] \circ b_{1}^{\circ n}\right)$ of $x \in \pi_{2 n} S L_{1} M U$ satisfies

$$
Q\left([1] \#\left([x] \circ b_{1}^{\circ n}\right)\right) \equiv \sum_{i=0}^{\infty}\left[c_{i}\right] \circ\left(b_{1}\right)^{\circ(i+2 n)} u^{2(i+n)} .
$$

In particular, $\widehat{Q}^{2 k}\left([1] \#\left([x] \circ b_{1}^{\circ n}\right)\right) \equiv\left[c_{k-n}\right] \circ b_{1}^{\circ(k+n)}$ in this quotient. 
Remark 4.4.4. We are working mod o-decomposables in $H_{*}\left(M U_{0}\right)$, and not in the entire Hopf ring, and so the right-hand side is not necessarily o-decomposable unless $c_{k-n}$ is.

Proof. By Corollary 4.4.2, we have

$$
Q\left([1] \#\left([x] \circ b_{1}^{\circ n}\right)\right) \equiv Q\left([x] \circ b_{1}^{\circ n}\right),
$$

and by Corollary 4.3.3 this is congruent to

$$
\sum_{i=0}^{\infty}\left[c_{i}\right] \circ\left(b_{1}\right)^{i+2 n} u^{2(i+n)} .
$$

In particular, taking coefficients of both sides gives us that

$$
\widehat{Q}^{2 k}\left([1] \#\left([x] \circ b_{1}^{\circ n}\right)\right) \equiv\left[c_{k-n}\right] \circ b_{1}^{\circ(k+n)}
$$

in this quotient.

Remark 4.4.5. The expression for $P(x)$ as a series in $\alpha$ is not unique due to the fact that it takes place in a quotient ring, and it is not immediately clear that the identity in this proposition is independent of this choice. However, any indeterminacy is a multiple of the identity $[2]_{F}(\alpha)=0$, whose image in the Hopf ring under the total unstable invariant translates into an identity in terms of the Ravenel-Wilson relations.

We can now apply the results of Johnson-Noel from Theorem 4.1.4, as well as Corollary 3.3.6 and Proposition 3.3.7.

Corollary 4.4.6. The Dyer-Lashof operations in $H_{*} S L_{1}(M U)$ satisfy

$$
\widehat{Q}^{10}\left([1] \#\left(\left[x_{2}\right] \circ b_{1}^{\circ 2}\right)\right) \equiv[1] \#\left(\left[x_{7}\right] \circ b_{1}^{\circ 7}\right)
$$

mod\#-decomposables, o-decomposables, and the ideal $\left(b_{2}, b_{3}, \ldots\right)$.

The Dyer-Lashof operations in $\pi_{*}\left(H \wedge_{M U} H\right)$ satisfy

$$
Q^{10}\left(\sigma x_{2}\right)=\sigma x_{7}
$$

Remark 4.4.7. We can take a brief pause to sketch why no map $M U \rightarrow B P$ can be given the structure of a map of $E_{7}$-algebras at the prime 2, extending [JN10]. If it could, then we can obtain a map of $E_{6}$-algebras $H \wedge_{M U} H \rightarrow H \wedge_{B P} H$, on homotopy given by a map of exterior algebras $\Lambda\left[\sigma x_{i}\right] \rightarrow \Lambda\left[\sigma x_{2^{i}-1}\right]$. However, this map would be zero on the element $\sigma x_{2}$ and nonzero on the element $\sigma x_{7}=Q^{10}\left(\sigma x_{2}\right)$. (Here we use that $Q^{10}$, on a class in degree 5 , is realized by an operation for $E_{6}$-algebras-see Remark 2.6.14.) This argument has been expanded in [Sen17]. 


\section{Calculations with $M U, H \mathbb{Z} / 2$, and $B P$}

In order to begin with more specific computations of secondary operations, we will use the following convenient definitions.

Definition 5.0.1. For a symbol $a$ and an integer $k$, we define $\mathbb{P}_{H}^{E_{n}}\left(a_{k}\right)$ to be the free $E_{n} H$-algebra $\mathbb{P}_{H}^{E_{n}}\left(S^{k}\right)$, writing $a_{k} \in \pi_{k} \mathbb{P}_{H}^{E_{n}}\left(a_{k}\right)$ for the generator represented by the unit map $S^{k} \rightarrow \mathbb{P}_{H}^{E_{n}}\left(S^{k}\right)$.

Similarly, we use the coproduct in $E_{n} H$-algebras to define

$$
\mathbb{P}_{H}^{E_{n}}\left(a_{k_{1}}, b_{k_{2}}, \ldots\right)=\mathbb{P}_{H}^{E_{n}}\left(a_{k_{1}}\right) \amalg \ldots \mathbb{P}_{H}^{E_{n}}\left(b_{k_{2}}\right) \amalg \cdots \cong \mathbb{P}_{H}^{E_{n}}\left(\vee S^{k_{i}}\right)
$$

for a sequence $\left(a_{k_{1}}, b_{k_{2}}, \ldots\right)$. If a generator has a known, fixed, degree, we will leave off the subscript.

Definition 5.0.2. Let $\mathcal{D}$ be the category of $E_{\infty} H$-algebras under $\mathbb{P}_{H}(x)$, where $x$ has degree 2 , and $\mathcal{D}^{n}$ the category of $E_{n} H$-algebras under $\mathbb{P}_{H}^{E_{n}}(x)$.

Let $C=\mathcal{D}^{ \pm}$and $C^{n}=\left(\mathcal{D}^{n}\right)^{ \pm}$as in Definition 2.2.1.

There are forgetful functors between these categories, using the compatible maps $\mathbb{P}_{H}^{E_{n}} S^{2} \rightarrow \mathbb{P}_{H}^{E_{m}} S^{2}$ that are adjoint to the units $S^{2} \rightarrow \mathbb{P}_{H}^{E_{m}} S^{2}$. The generator of $H_{2} M U \cong$ $\mathbb{Z} / 2$ determines a map $\mathbb{P}_{H}(x) \rightarrow H \wedge M U$ up to equivalence, lifting it to an object of C.

\subsection{Power operations for $M U$}

The 2-primary power operations in $H_{*} M U$ are known by work of Kochman [Koc73], but the following closed-form formula is due to Priddy.

Theorem 5.1.1 ([Pri75]). The Dyer-Lashof operations in $H_{*} M U \cong H_{*} B U$ are determined by the following identity:

$$
\sum Q^{j} b_{k}=\left(\sum_{n=k}^{\infty} \sum_{u=0}^{k}\left(\begin{array}{c}
n-k+u-1 \\
u
\end{array}\right) b_{n+u} b_{k-u}\right)\left(\sum_{n=0}^{\infty} b_{n}\right)^{-1}
$$

Here $b_{0}=1$ by convention. In particular, we have

$$
\sum Q^{j} b_{1}=\left(\sum_{n=1}^{\infty}\left(b_{n} b_{1}+(n-1) b_{n+1}\right)\right)\left(\sum_{n=0}^{\infty} b_{n}\right)^{-1} .
$$

This allows the following direct computation. (Compare [Pri75, 2.5], which carries out this computation for $M O$ ). 
Proposition 5.1.2. We have the following Dyer-Lashof operations in $H_{*} M U$ :

$$
\begin{aligned}
Q^{2} b_{1} & =b_{1}^{2} \\
Q^{4} b_{1} & =b_{3}+b_{1} b_{2}+b_{1}^{3} \\
Q^{6} b_{1} & =b_{1}^{4} \\
Q^{8} b_{1} & =b_{5}+b_{1} b_{4}+b_{2} b_{3}+b_{1}^{2} b_{3}+b_{1} b_{2}^{2}+b_{1}^{3} b_{2}+b_{1}^{5} \\
Q^{10} b_{1} & =b_{3}^{2}+b_{1}^{2} b_{2}^{2}+b_{1}^{6} \\
Q^{6} b_{2} & =b_{5}+b_{1} b_{4}+b_{2} b_{3}+b_{1} b_{2}^{2} \\
Q^{10} b_{2} & =b_{1}^{2} b_{5}+b_{1}^{3} b_{4}+b_{1}^{2} b_{2} b_{3}+b_{1}^{3} b_{2}^{2}
\end{aligned}
$$

In particular, the following identities hold:

$$
\begin{aligned}
0 & =Q^{6} b_{1}+b_{1}^{4} \\
0 & =Q^{10} b_{1}+\left(Q^{4} b_{1}\right)^{2} \\
Q^{6} b_{2} & =Q^{8} b_{1}+b_{1}^{2} Q^{4} b_{1} \\
0 & =Q^{10} b_{2}+b_{1}^{2} Q^{6} b_{2}
\end{aligned}
$$

\subsection{Power operations for $H$}

The power operations in the dual Steenrod algebra are known by work of Steinberger.

Theorem 5.2.1 ([BMMS86, III.2.2, III.2.4]). The 2-primary Dyer-Lashof operations in the dual Steenrod algebra satisfy the following identities:

$$
\begin{gathered}
1+\xi_{1}+Q^{1} \xi_{1}+Q^{2} \xi_{1}+Q^{3} \xi_{1}+\cdots=\left(1+\xi_{1}+\xi_{2}+\ldots\right)^{-1} \\
Q^{s} \bar{\xi}_{i}= \begin{cases}Q^{s+2^{i}-2} \xi_{1} & \text { ifs } \equiv 0,-1 \quad \bmod 2^{i}, \\
0 & \text { otherwise. }\end{cases} \\
Q^{2^{i} \bar{\xi}_{i}}=\bar{\xi}_{i+1}
\end{gathered}
$$

This, again, allowed direct computation.

Proposition 5.2.2 ([BMMS86, III.5]). We have the following Dyer-Lashof operations in the 2-primary dual Steenrod algebra:

$$
\begin{aligned}
Q^{2} \bar{\xi}_{1} & =\bar{\xi}_{2} \\
Q^{3} \bar{\xi}_{1} & =\bar{\xi}_{1}^{4} \\
Q^{4} \bar{\xi}_{1} & =\bar{\xi}_{1}^{2} \bar{\xi}_{2} \\
Q^{5} \bar{\xi}_{1} & =\bar{\xi}_{2}^{2} \\
Q^{16} \bar{\xi}_{4} & =\bar{\xi}_{5}
\end{aligned}
$$


In particular, the Cartan formula implies that the following identities hold:

$$
\begin{aligned}
& 0=Q^{6} \bar{\xi}_{1}^{2}+\bar{\xi}_{1}^{8} \\
& 0=Q^{8} \bar{\xi}_{1}^{2}+\bar{\xi}_{1}^{4} Q^{4} \bar{\xi}_{1}^{2} \\
& 0=Q^{10} \bar{\xi}_{1}^{2}+\left(Q^{4} \bar{\xi}_{1}^{2}\right)^{2}
\end{aligned}
$$

Remark 5.2.3. While the identity $Q^{16} \bar{\xi}_{4}=\bar{\xi}_{5}$ is valid, the results of this paper only require us to know the easier statement that $Q^{16} \xi_{4} \equiv \xi_{5} \bmod$ decomposable elements.

\subsection{Functional operations for $M U \rightarrow H \mathbb{Z} / 2$}

Recall that the category $C$ is the category of $E_{\infty} H$-algebras under $\mathbb{P}_{H}(x)$, where $x$ has degree 2 .

Theorem 5.3.1. Consider the maps

$$
\mathbb{P}_{H}\left(x, z_{14}\right) \stackrel{\bar{Q}}{\rightarrow} \mathbb{P}_{H}\left(x, y_{4}\right) \stackrel{f}{\rightarrow} H \wedge M U \stackrel{p}{\rightarrow} H \wedge H
$$

in the category $C$, where $\bar{Q}$ sends $z_{14}$ to $Q^{10} \underline{y}_{4}+x^{2} Q^{6} y_{4}$ and $f$ sends $\left(x, y_{4}\right)$ to $\left(b_{1}, b_{2}\right)$. Then a functional homotopy operation $\langle p, f, \bar{Q}\rangle$ is defined in $\mathbb{P}_{H}(x)$-algebras and satisfies

$$
\langle p, f, \bar{Q}\rangle \equiv \xi_{4}
$$

mod decomposables.

Proof. The identities $Q^{10} b_{2}+b_{1}^{2} Q^{6} b_{2}=0$ and $p\left(b_{2}\right)=0$ ensure that there is a homotopy commutative diagram of $E_{\infty} \mathbb{P}_{H}(x)$-algebras over $H$ :

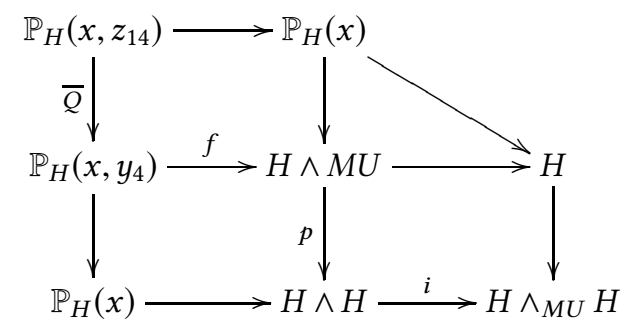

In particular, $\bar{Q}$ is a map of augmented objects and $H \wedge_{M U} H$ is a pointed object, ensuring that the secondary operation is defined. As a result, we can define $\langle p, f, \bar{Q}\rangle$ and apply the Peterson-Stein formula of Proposition 2.3.5 to find that there is an identity

$$
\langle i, p, f\rangle \bar{Q}=i\langle p, f, \bar{Q}\rangle .
$$

(Note that there is no inversion in this Peterson-Stein formula because the target group is a vector space over $\mathbb{F}_{2}$.)

The bracket $\langle i, p, f\rangle$ takes $y_{4}$, which maps under $f$ to the Hurewicz image $b_{2}$ of $x_{2} \in \pi_{2} M U$, to the suspension class $\sigma b_{2}$ up to indeterminacy by Proposition 2.7.5. 
The operation $\bar{Q}$ sends this to $Q^{10}\left(\sigma b_{2}\right)+x^{2} Q^{6}\left(\sigma b_{2}\right)=Q^{10}\left(\sigma b_{2}\right)$ because $x$ acts by 0 on $H \wedge_{M U} H$. Then Corollary 4.4.6 implies that $Q^{10}\left(\sigma b_{2}\right) \equiv \sigma x_{7} \bmod$ decomposables, and the proof of Proposition 2.7.6 shoes that $\sigma x_{7} \equiv i\left(\xi_{4}\right) \bmod$ decomposables. Thus we find that $i\langle p, f, \bar{Q}\rangle=\langle i, p, f\rangle \bar{Q} \equiv i\left(\xi_{4}\right) \bmod$ decomposables.

The indeterminacy in the functional homotopy operation $\langle p, f, \bar{Q}\rangle$ consists of elements in the image of $p$ and elements in the image of $\sigma \bar{Q}$, which are of the form $Q^{10}\left(y_{5}^{\prime}\right)+\xi_{1}^{2} Q^{6}\left(y_{5}^{\prime}\right)$. However, there are no indecomposables in the image of $p$ and no indecomposables in the dual Steenrod algebra in degree 5, and so the indeterminacy consists completely of decomposable elements. The map $i$ is an isomorphism on homotopy in degree 15 mod decomposables, and hence $\langle p, f, \bar{Q}\rangle \equiv \xi_{4}$ mod decomposables.

\subsection{A secondary operation in the dual Steenrod algebra}

Proposition 5.4.1. Suppose that $R$ is an $E_{12} H$-algebra and $x \in \pi_{2}(R)$. Define the following classes:

$$
\begin{aligned}
y_{5} & =Q^{3} x \\
y_{7} & =Q^{5} x \\
y_{9} & =Q^{7} x \\
y_{13} & =Q^{11} x \\
y_{8} & =Q^{6} x+x^{4} \\
y_{10} & =Q^{8} x+x^{2} Q^{4} x \\
y_{12} & =Q^{10} x+\left(Q^{4} x\right)^{2}
\end{aligned}
$$

Then there is an identity

$$
\begin{aligned}
0= & Q^{20} y_{10}+Q^{18} y_{12}+Q^{17} y_{13}+x^{4}\left(Q^{12} y_{10}\right)+y_{9}^{2}\left(Q^{4} x\right)^{2}+ \\
& y_{7}^{2} Q^{9} Q^{5} x+y_{8}^{2} Q^{8} Q^{4} x+\left(Q^{9} y_{9}\right)\left(Q^{4} x\right)^{2}+\left(Q^{10} y_{8}\right)\left(Q^{4} x\right)^{2}+ \\
& y_{5}^{2}\left(Q^{11} Q^{7} x+Q^{10} Q^{8} x+x^{4} Q^{6} Q^{4} x\right)
\end{aligned}
$$

Proof. The following table breaks this down term-by-term, substituting in the values 
of the $y_{i}$.

$$
\begin{aligned}
Q^{20} y_{10} & =Q^{20} Q^{8} x+Q^{20}\left(x^{2} Q^{4} x\right) \\
Q^{18} y_{12} & =Q^{18} Q^{10} x+Q^{18}\left(\left(Q^{4} x\right)^{2}\right) \\
Q^{17} y_{13} & =Q^{17} Q^{11} x \\
x^{4}\left(Q^{12} y_{10}\right) & =x^{4}\left(Q^{12} Q^{8} x\right)+x^{8} Q^{8} Q^{4} x+x^{4}\left(Q^{3} x\right)^{2} Q^{6} Q^{4} x \\
y_{9}^{2}\left(Q^{4} x\right)^{2} & =\left(Q^{7} x\right)^{2}\left(Q^{4} x\right)^{2} \\
y_{7}^{2} Q^{9} Q^{5} x & =\left(Q^{5} x\right)^{2} Q^{9} Q^{5} x \\
y_{8}^{2} Q^{8} Q^{4} x & =\left(Q^{6} x\right)^{2} Q^{8} Q^{4} x+x^{8} Q^{8} Q^{4} x \\
\left(Q^{9} y_{9}\right)\left(Q^{4} x\right)^{2} & =\left(Q^{9} Q^{7} x\right)\left(Q^{4} x\right)^{2} \\
\left(Q^{10} y_{8}\right)\left(Q^{4} x\right)^{2} & =\left(Q^{10} Q^{6} x\right)\left(Q^{4} x\right)^{2} \\
y_{5}^{2}\left(Q^{11} Q^{7} x\right) & =\left(Q^{3} x\right)^{2}\left(Q^{11} Q^{7} x\right) \\
y_{5}^{2}\left(Q^{10} Q^{8} x\right) & =\left(Q^{3} x\right)^{2} Q^{10} Q^{8} x \\
y_{5}^{2}\left(x^{4} Q^{6} Q^{4} x\right) & =\left(Q^{3} x\right)^{2}\left(x^{4} Q^{6} Q^{4} x\right)
\end{aligned}
$$

The reader who is interested in ensuring that these cancel is encouraged to do so with the aid of a pen. To assist this, we list the following needed identities deduced from the Cartan formula, Adem relations, and instability relations where appropriate.

$$
\begin{aligned}
Q^{20} Q^{8} x= & Q^{18} Q^{10} x+Q^{17} Q^{11} x \\
Q^{20}\left(x^{2} Q^{4} x\right)= & x^{4} Q^{16} Q^{4} x+\left(Q^{3} x\right)^{2} Q^{14} Q^{4} x+\left(Q^{4} x\right)^{2} Q^{12} Q^{4} x+ \\
& \left(Q^{5} x\right)^{2} Q^{10} Q^{4} x+\left(Q^{6} x\right)^{2} Q^{8} Q^{4} x+\left(Q^{7} x\right)^{2}\left(Q^{4} x\right)^{2} \\
Q^{18}\left(\left(Q^{4} x\right)^{2}\right)= & 0 \\
x^{4} Q^{16} Q^{4} x= & x^{4} Q^{12} Q^{8} x \\
\left(Q^{3} x\right)^{2} Q^{14} Q^{4} x= & \left(Q^{3} x\right)^{2} Q^{11} Q^{7} x+\left(Q^{3} x\right)^{2} Q^{10} Q^{8} x \\
\left(Q^{4} x\right)^{2} Q^{12} Q^{4} x= & \left(Q^{4} x\right)^{2} Q^{10} Q^{6} x+\left(Q^{4} x\right)^{2} Q^{9} Q^{7} x \\
\left(Q^{5} x\right)^{2} Q^{10} Q^{4} x= & \left(Q^{5} x\right)^{2} Q^{9} Q^{5} x
\end{aligned}
$$

To apply $Q^{r}$ to an element in degree $s$, as well as make use of the Adem relations, Cartan formula, and instability relations, we require the presence of an $E_{n}$-algebra for $n \geq r-s+2$. The greatest value of $n$ required from the equations above is when we take $Q^{20} y_{10}$, and in particular use additivity for $Q^{20}$, which requires an $E_{12}$-algebra.

We can use this relation to build secondary operations.

Proposition 5.4.2. Suppose $n \geq 12$ and let $R$ be an object of $C^{n}$, corresponding to an $E_{n} H$-algebra with an element $x \in \pi_{2}(R)$, such that the classes $y_{i}$ of Proposition 5.4.1 vanish. Then there is a secondary operation on $x$ given by $\langle x, Q \stackrel{h}{\leftrightarrow} R\rangle \in \pi_{31} R$. The indeterminacy in this secondary operation consists of elements of the form

$$
Q^{20} y_{11}^{\prime}+Q^{18} y_{13}^{\prime}+Q^{17} y_{14}^{\prime}
$$


and decomposables. This secondary operation is preserved by the forgetful functors $C^{m} \rightarrow C^{n}$ for $m>n$.

Proof. For any $n \geq 12$, Proposition 5.4.1 describes a relation between homotopy operations, in the form of a homotopy commutative diagram of $E_{n} H$-algebras



adjoint to a commutative diagram of $E_{n}$-algebras under $\mathbb{P}_{H}^{E_{n}}(x)$ of the form

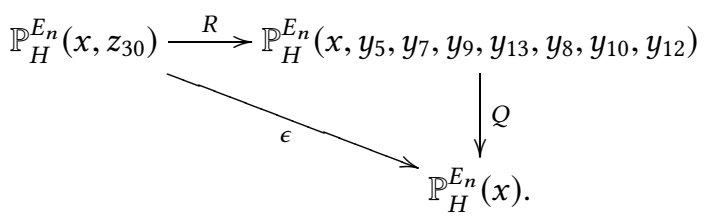

Here the maps $Q$ and $R$ are defined by the equations of Proposition 5.4.1. The map $R$ is a map of augmented objects, the domain by the map $\epsilon$ sending $z_{30}$ to 0 and the range by the map sending all $y_{i}$ to zero. In particular, the homotopy commutativity of the above diagrams shows that there exists a tethering $Q \stackrel{h}{\leftrightarrow} R$ in the category $C^{n}$.

The indeterminacy in this secondary operation consists of elements in the image of the suspended operation

$$
\sigma R: \mathbb{P}_{H}^{E_{n}}\left(x, z_{31}^{\prime}\right) \rightarrow \mathbb{P}_{H}^{E_{n}}\left(x, y_{6}^{\prime}, y_{8}^{\prime}, y_{10}^{\prime}, y_{14}^{\prime}, y_{9}^{\prime}, y_{11}^{\prime}, y_{12}^{\prime}\right) .
$$

Proposition 2.6.13 implies that $\sigma R$ is given by

$$
z_{31}^{\prime} \mapsto Q^{20} y_{11}^{\prime}+Q^{18} y_{13}^{\prime}+Q^{17} y_{14}^{\prime}+x^{4}\left(Q^{12} y_{11}^{\prime}\right)+\left(Q^{9} y_{10}^{\prime}\right)\left(Q^{4} x\right)^{2}+\left(Q^{10} y_{9}^{\prime}\right)\left(Q^{4} x\right)^{2},
$$

since the other terms involve binary products that map to zero. However, the terms other than $Q^{20} y_{11}^{\prime}+Q^{18} y_{13}^{\prime}+Q^{17} y_{14}^{\prime}$ always take decomposable values.

Proposition 5.4.3. In the 2-primary dual Steenrod algebra

$$
H_{*} H \cong \mathbb{F}_{2}\left[\xi_{1}, \xi_{2}, \ldots\right],
$$

viewed as the homotopy of the $H$-algebra $H \wedge H$, the bracket $\left\langle\xi_{1}^{2}, Q, R\right\rangle$ is defined, and the indeterminacy is zero mod decomposables.

Proof. The Cartan formula for Dyer-Lashof operations immediately implies that $Q^{2 k+1}\left(\xi_{1}^{2}\right)=$ 0 for all $k$. The remaining identities

$$
\begin{aligned}
& 0=Q^{6} \xi_{1}^{2}+\xi_{1}^{8} \\
& 0=Q^{8} \xi_{1}^{2}+\xi_{1}^{4} Q^{4} \xi_{1}^{2} \\
& 0=Q^{10} \xi_{1}^{2}+\left(Q^{4} \xi_{1}^{2}\right)^{2}
\end{aligned}
$$


were determined in Proposition 5.2.2. Therefore, $\xi_{1}^{2} Q=0$ and the bracket $\left\langle\xi_{1}^{2}, Q, R\right\rangle$ is defined.

We now consider the indeterminacy. The indeterminacy is generated by adding the results of degree-29 homotopy operations applied to $\xi_{1}^{2}$, Dyer-Lashof operations applied to elements in degrees 11,13, and 14, and decomposables. Proposition 2.6.12 showed that all nonconstant homotopy operations are generated by multiplication, addition, and the operations $Q^{n}$, all of which preserve decomposables. The dual Steenrod algebra contains no indecomposables in degrees 11,13 , and 14 , and so any operation applied to such an element is decomposable.

The operations $Q$ and $R$, while complex, can be related to simpler operations using the following diagram.

Proposition 5.4.4. Consider the maps

$$
\begin{aligned}
& \mu: \mathbb{P}_{H}\left(x, y_{5}, y_{7}, y_{9}, y_{13}, y_{8}, y_{10}, y_{12}\right) \rightarrow \mathbb{P}_{H}\left(x, y_{4}\right) \\
& \nu: \mathbb{P}_{H}\left(x, z_{30}\right) \rightarrow \mathbb{P}_{H}\left(x, z_{14}\right) \\
& \alpha: \mathbb{P}_{H}\left(x, z_{30}\right) \rightarrow \mathbb{P}_{H}\left(x, z_{15}\right) \\
& \beta: \mathbb{P}_{H}\left(x, z_{15}\right) \rightarrow \mathbb{P}_{H}\left(x, y_{4}\right)
\end{aligned}
$$

of augmented objects, defined by the identities

$$
\begin{aligned}
\mu\left(y_{i}\right) & =0 \text { for } i \neq 10 \\
\mu\left(y_{10}\right) & =Q^{6} y_{4} \\
\nu\left(z_{30}\right) & =Q^{16} z_{14} \\
\alpha\left(z_{30}\right) & =\left(z_{15}\right)^{2} \\
\beta\left(z_{15}\right) & =Q^{3} x Q^{6} y_{4}
\end{aligned}
$$

Then there is an identity $\mu R=\bar{Q} v+\beta \alpha$ and a homotopy commutative diagram in $C$ of the form

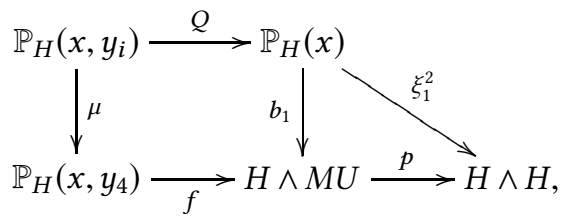

where $f$ and $\bar{Q}$ are from Theorem 5.3.1.

Proof. It is classical that the map $p: H_{2} M U \rightarrow H_{2} H$ takes $b_{1}$ to $\xi_{1}^{2}$, making the righthand triangle commute.

To verify that the map $\mu$ makes the square diagram commute in the homotopy 
category, we need to know that the Dyer-Lashof operations on $b_{1}$ satisfy

$$
\begin{aligned}
0 & =Q^{3} b_{1} \\
0 & =Q^{5} b_{1} \\
0 & =Q^{7} b_{1} \\
0 & =Q^{11} b_{1} \\
0 & =Q^{6} b_{1}+b_{1}^{4} \\
0 & =Q^{10} b_{1}+\left(Q^{4} b_{1}\right)^{2} \\
Q^{6} b_{2} & =Q^{8} b_{1}+b_{1}^{2} Q^{4} b_{1}
\end{aligned}
$$

The odd operations vanish automatically because $H_{*} M U$ is concentrated in even degrees, and the remaining three identities were proven in Proposition 5.1.2.

Finally we need to verify the identity $\bar{Q} v=\beta \alpha+\mu R$. Using the definition of $\mu$ and the formula from Proposition 5.4.1 for $R$, we find that

$$
\mu\left(R\left(z_{30}\right)\right)=Q^{20} Q^{6} y_{4}+x^{4} Q^{12} Q^{6} y_{4}
$$

In the Adem relation $Q^{20} Q^{6}=Q^{16} Q^{10}+Q^{14} Q^{12}+Q^{13} Q^{13}$, the last two terms automatically vanish on classes in degree four. Therefore, we can continue to simplify, finding

$$
\begin{aligned}
\mu\left(R\left(z_{30}\right)\right) & =Q^{16} Q^{10} y_{4}+x^{4} Q^{12} Q^{6} y_{4} \\
& =Q^{16} Q^{10} y_{4}+Q^{16}\left(x^{2} Q^{6} y_{4}\right)+\left(Q^{3} x\right)^{2}\left(Q^{6} y_{4}\right)^{2} \\
& =Q^{16}\left(Q^{10} y_{4}+x^{2} Q^{6} y_{4}\right)+\left(Q^{3} x Q^{6} y_{4}\right)^{2} \\
& =Q^{16}\left(\bar{Q}\left(z_{14}\right)\right)+\beta\left(z_{15}\right)^{2} \\
& =\bar{Q} v\left(z_{30}\right)+\beta \alpha\left(z_{30}\right),
\end{aligned}
$$

as desired.

Corollary 5.4.5. In the dual Steenrod algebra, any element in the bracket $\left\langle\xi_{1}^{2}, Q, R\right\rangle$ is congruent to $\xi_{5}$ mod decomposables.

Proof. We first observe that three types of elements in degree 31 are decomposable in the dual Steenrod algebra.

- The first are elements in the image of $p: H_{*} M U \rightarrow H_{*} H$ : the only indecomposable element in the image of $p$ is $1 \in H_{0} H$.

- The second are elements in the image of $\sigma R$, which (as in Proposition 5.4.2) consists of multiples of Dyer-Lashof operations applied to elements in degrees 11, 13, and 14. Degrees 11, 13 and 14 contain no indecomposables, and so the Cartan formula for Dyer-Lashof operations implies that any elements in the image of $\sigma R$ are decomposable. 
- The third are elements in the image of $\sigma(\bar{Q} v)$ or $\sigma(\beta \alpha)$, both of which are multiples of Dyer-Lashof operations applied to classes in degree 5 . Degree 5 contains no indecomposables, and thus similarly the images of these elements are indecomposable.

Multiple applications of Proposition 2.3.5 and Proposition 2.4.3 give us the following string of identities.

$$
\begin{aligned}
\left\langle\xi_{1}^{2}, Q, R\right\rangle & =\left\langle p b_{1}, Q, R\right\rangle \\
& \subset\left\langle p, b_{1} Q, R\right\rangle \\
& =\langle p, f \mu, R\rangle \\
& \supset\langle p, f, \mu R\rangle \\
& =\langle p, f, \bar{Q} v+\beta \alpha\rangle \\
& \subset\langle p, f, \bar{Q} v\rangle+\langle p, f, \beta \alpha\rangle \\
& \supset\langle p, f, \bar{Q}\rangle v+\langle p, f, \beta\rangle \alpha .
\end{aligned}
$$

We note that in all of these brackets, the indeterminacy is contained in the three types mentioned above: the image of $p$, the image of $\sigma R$, and the images of $\sigma(\bar{Q} v)$ or $\sigma(\beta \alpha)$. It suffices to check at the local maxima for indeterminacy in this chain of containments: the brackets $\left\langle p, b_{1} Q, R\right\rangle$ and $\langle p, f, \bar{Q} v\rangle+\langle p, f, \beta \alpha\rangle$. Therefore, if we work mod decomposables we get unambiguous values and these containments become equalities. We find

$$
\left\langle\xi_{1}^{2}, Q, R\right\rangle \equiv\langle p, f, \bar{Q}\rangle v+\langle p, f, \beta\rangle \alpha .
$$

By Theorem 5.3.1, we have

$$
\langle p, f, \bar{Q}\rangle\left(v z_{30}\right)=Q^{16}\left(\langle p, f, \bar{Q}\rangle\left(z_{14}\right)\right) \equiv Q^{16} \xi_{4} \equiv \xi_{5}
$$

mod decomposables. On the other hand,

$$
\langle p, f, \beta\rangle \alpha\left(z_{30}\right)=\left(\langle p, f, \beta\rangle\left(z_{15}\right)\right)^{2}
$$

which is automatically decomposable. Therefore, every element in $\left\langle\xi_{1}^{2}, Q, R\right\rangle$ is congruent to $\xi_{5} \bmod$ decomposables.

Theorem 5.4.6. Suppose that $n \geq 12$ and $R$ is an $E_{n}$ ring spectrum with a mapg: $R \rightarrow$ $H$ and an element $x \in H_{2}(R)$ such that $g(x)=\xi_{1}^{2}$ in $H_{2} H$. If the element $x$ makes the classes $y_{i}$ of Proposition 5.4.1 zero, then the map $H_{31} R \rightarrow H_{31} H$ has $\xi_{5}$ in its image mod decomposables.

In particular, if $H_{*} R \rightarrow H_{*} H$ is injective through degree 13, this result holds.

Proof. Under these conditions, $H \wedge R \rightarrow H \wedge H$ is a map of $E_{n} H$-algebras, and (up to equivalence) the map $\mathbb{P}_{H}^{E_{n}}(x) \rightarrow H \wedge H$ lifts to a map $\mathbb{P}_{H}^{E_{n}}(x) \rightarrow H \wedge R$. Thus, $H \wedge R \rightarrow H \wedge H$ can be lifted to a map in $C^{n}$ which, on homotopy groups, gives the $\operatorname{map} g: H_{*} R \rightarrow H_{*} H$.

Then the secondary operation $\langle x, Q, R\rangle$ is defined and the map $g$ carries $\langle x, Q, R\rangle$ into a subset of $\left\langle\xi_{1}^{2}, Q, R\right\rangle$, all of whose elements are congruent to $\xi_{5} \bmod$ decomposables. 


\subsection{Application to the Brown-Peterson spectrum}

Using Theorem 5.4.6, we can now exclude the existence of $E_{n}$-algebra structures on spectra related to the Brown-Peterson spectrum. We first recall the homology of the Brown-Peterson spectrum, dual to the cohomology described in [BP66].

Proposition 5.5.1. The Brown-Peterson spectrum $B P$ is connective, with $\pi_{0} B P \cong \mathbb{Z}_{(2)}$. The map $B P \rightarrow \mathrm{HF}_{2}$ induces an inclusion $H_{*} B P \hookrightarrow H_{*} H_{\mathbb{F}_{2}}$ whose image is the subalgebra

$$
\mathbb{F}_{2}\left[\xi_{1}^{2}, \xi_{2}^{2}, \ldots\right] \subset \mathbb{F}_{2}\left[\xi_{1}, \xi_{2}, \ldots\right]
$$

of the dual Steenrod algebra. The image in positive degrees consists entirely of decomposables.

Similarly, we have truncated Brown-Peterson spectra $\mathrm{BP}\langle k\rangle$ and their generalized versions.

Proposition 5.5.2 ([LN14, 4.3]). Any generalized truncated Brown-Peterson spectrum $B P\langle k\rangle$ is connective, with $\pi_{0} B P\langle k\rangle=\mathbb{Z}_{(2)}$. The map $B P\langle k\rangle \rightarrow \mathrm{HF}_{2}$ induces an inclusion $H_{*} B P \hookrightarrow H_{*} \mathrm{HF}_{2}$ whose image is the subalgebra

$$
\mathbb{F}_{2}\left[\xi_{1}^{2}, \xi_{2}^{2}, \ldots \xi_{k+1}^{2}, \xi_{k+2}, \xi_{k+3}, \ldots\right] \subset \mathbb{F}_{2}\left[\xi_{1}, \xi_{2}, \ldots\right]
$$

of the dual Steenrod algebra. The image in positive degrees consists entirely of decomposables until dimension $2^{k+2}-2$.

In particular, the element $\xi_{5}$ is not in the image mod decomposables for $k \geq 4$. These spectra are also of finite type, and their 2-adic completions have the same homology groups.

By considering the cohomology in degree zero, we find that there is a unique nontrivial map of spectra $B P \rightarrow H \mathbb{F}_{2}$, and similarly for $B P\langle k\rangle$. (At odd primes, this map is unique up to scalar.) As $E_{n}$-algebras have Postnikov towers, there is the following consequence.

Corollary 5.5.3. If BP or $B P\langle k\rangle$ admits the structure of an $E_{n}$-algebra, then the unique nontrivial map to $\mathrm{HF}_{2}$ lifts to a map of $E_{n}$-algebras.

We can now apply Theorem 5.4.6.

Theorem 5.5.4. The 2-local Brown-Peterson spectrum BP, the (generalized) truncated Brown-Peterson spectra $B P\langle k\rangle$ for $k \geq 4$, and their 2-adic completions do not admit the structure of $E_{n}$-algebras for any $12 \leq n \leq \infty$.

Remark 5.5.5. The above results can also be applied to appropriate truncations in the Postnikov tower for $B P$.

\section{A Power operations in the Lazard ring}

In this section we will extend Johnson-Noel's proof of Theorem 4.1 .4 to a proof that works in torsion-free quotients of the Lazard ring. The following calculations are specialized to the prime 2 . 
The power operation $P$ of Section 4.1 takes the form of a natural transformation

$$
P: M U^{2 n}(X) \rightarrow M U^{4 n}\left(X \times B \Sigma_{2}\right) \hookrightarrow M U^{4 n}(X) \llbracket \alpha \rrbracket /[2]_{F}(\alpha) .
$$

Writing the 2-series as

$$
[2]_{F}(\alpha)=\alpha \cdot\langle 2\rangle_{F}(\alpha),
$$

we have the following properties.

- The identity $P(u v)=P(u) P(v)$ holds.

- The identity $P(u) \equiv u^{2}$ holds $\bmod \alpha$.

- The identity $P(u+v) \equiv P(u)+P(v)$ holds $\bmod \langle 2\rangle_{F}(\alpha)$. In particular, $P$ becomes a ring homomorphism in this quotient.

- On the canonical orientation $x \in \widetilde{M U}^{2}\left(\mathbb{C P}^{\infty}\right)$, we have $P(x)=x\left(x+{ }_{F} \alpha\right)$.

Let $L=M U^{*}$ be the Lazard ring, and define $g(x, \alpha)=x\left(x+{ }_{F} \alpha\right)$ in the power series ring $L \llbracket x, \alpha \rrbracket$. Applying the identities for $P$ to the spaces $\left(\mathbb{C} \mathbb{P}^{\infty}\right)^{n}$ and the natural maps between them, we deduce the following.

Proposition A.0.1. The map $P$ induces a ring homomorphism

$$
\Psi: L \rightarrow L \llbracket \alpha \rrbracket /\langle 2\rangle_{F}(\alpha)
$$

and the power series $g(x, \alpha)$ defines an isogeny $F \rightarrow \Psi^{*} F$ :

$$
g(x, \alpha) \underset{\Psi^{*} F}{+} g(y, \alpha) \equiv g\left(x+{ }_{F} y, \alpha\right) .
$$

The rings $L$ and $L \llbracket \alpha \rrbracket /\langle 2\rangle_{F}(\alpha)$ are torsion-free, and so the formal group laws $F$ and $\Psi^{*} F$ have logarithms:

$$
\ell_{F}(x)=\sum \frac{\mathbb{C P}^{n-1} x^{n}}{n} \quad \ell_{\Psi^{*} F}(x, \alpha)=\sum \frac{\Psi\left(\mathbb{C P}^{n-1}\right) x^{n}}{n}
$$

By choosing any lifts of $\Psi\left(\mathbb{C P}^{n}\right)$ to $L \llbracket \alpha \rrbracket$, we can view these formulas as defining power series $\left(\ell_{F}\right)^{\prime}(x) \in L \llbracket x \rrbracket$ and $\left(\ell_{\Psi^{*} F}\right)^{\prime}(x, \alpha) \in L \llbracket x, \alpha \rrbracket$.

Taking derivatives of (A.1) with respect to $y$ and evaluating at $y=0$, we find

$$
\frac{g^{\prime}(0, \alpha)}{\left(\ell_{\Psi^{*} F}\right)^{\prime}(g(x, \alpha), \alpha)} \equiv \frac{g^{\prime}(x, \alpha)}{\left(\ell_{F}\right)^{\prime}(x)}
$$

in $L \llbracket x, \alpha \rrbracket /\langle 2\rangle_{F}(\alpha)$, and thus

$$
g^{\prime}(x, \alpha)\left(\ell_{\Psi^{*} F}\right)^{\prime}(g(x, \alpha), \alpha)=\alpha \cdot\left(\ell_{F}\right)^{\prime}(x, \alpha)+h(x, \alpha) \cdot\langle 2\rangle_{F}(\alpha)
$$

for some power series $h(x, \alpha) \in L \llbracket x, \alpha \rrbracket$.

We now substitute $x=\alpha y$ and observe that

$$
g(\alpha y, \alpha)=\alpha^{2} k(y, \alpha)
$$


where the power series $k(y, \alpha)$ has the form $y+O\left(y^{2}\right)$. Hence, there a composition inverse: a series $k^{-1}(y, \alpha)$ of the same form such that $k\left(k^{-1}(y, \alpha), \alpha\right)=y$.

Substituting $x=\alpha y$ in to (A.2), we obtain an identity

$$
\alpha k^{\prime}(y, \alpha)\left(\ell_{\Psi^{*} F}\right)^{\prime}\left(\alpha^{2} k(y, \alpha), \alpha\right)=\alpha \cdot\left(\ell_{F}\right)^{\prime}(\alpha y)+h(\alpha y, \alpha) \cdot\langle 2\rangle_{F}(\alpha)
$$

which can be simplified to the statement

$$
\left(\ell_{\Psi^{*} F}\right)^{\prime}\left(\alpha^{2} y, \alpha\right)=\left(\ell_{F}\right)^{\prime}\left(\alpha k^{-1}(y, \alpha)\right)\left(k^{-1}\right)^{\prime}(y, \alpha)+\tilde{h}(y, \alpha) \cdot\langle 2\rangle_{F}(\alpha)
$$

for some series $\tilde{h}(y, \alpha) \in L \llbracket y, \alpha \rrbracket$. If we write $f_{n}(\alpha)$ for the coefficient of $y^{n}$ in $\left(\ell_{F}\right)^{\prime}\left(\alpha k^{-1}(y, \alpha)\right)\left(k^{-1}\right)^{\prime}(y, \alpha)$, we then find that

$$
\Psi\left(\mathbb{C P}^{n}\right) \alpha^{2 n}=f_{n}(\alpha)+\tilde{h}_{n}(\alpha) \cdot\langle 2\rangle_{F}(\alpha)
$$

for some series $\tilde{h}_{n}(\alpha)$. If $f: L \rightarrow S$ is any ring homomorphism, there is a degree- $2 n$ polynomial $h_{n}(\alpha) \in S[\alpha]$ such that

$$
f_{n}(\alpha)-h_{n}(\alpha) \cdot\langle 2\rangle_{F}(\alpha) \equiv f\left(\mathbb{C P}^{n}\right)^{2}
$$

in $S \llbracket \alpha \rrbracket /\left(\alpha^{2 n+1}\right)$. If 2 is not a zero divisor in the ring $S$, this determines $h_{n}(\alpha)$ uniquely and so it can be calculated in $S$. We deduce that

$$
f\left(P\left(\mathbb{C P}^{n}\right)\right) \equiv \alpha^{-2 n}\left(f_{n}(\alpha)-h_{n}(\alpha) \cdot\langle 2\rangle_{F}(\alpha)\right)
$$

in $S \llbracket \alpha \rrbracket /[2]_{F}(\alpha)$.

In particular, we may take $S=\mathbb{Z}\left[v_{3}\right] /\left(v_{3}^{2}\right)$, which has logarithm $x+\frac{v_{3}}{2} x^{8}$. We can then expand out the definitions in this ring.

$$
\begin{aligned}
x+{ }_{F} y & =x+y+\frac{v_{3}}{2}\left(x^{8}+y^{8}-(x+y)^{8}\right) \\
\langle 2\rangle_{F}(\alpha) & =2-127 v_{3} \alpha^{7} \\
\left(\ell_{F}\right)^{\prime}(x) & =1+4 v_{3} x^{7} \\
g(x, \alpha) & =\alpha x+x^{2}+\frac{v_{3}}{2}\left(\alpha^{8}+x^{8}-(\alpha+x)^{8}\right) \\
& =\alpha x+\left(1-4 v_{3} \alpha^{7}\right) x^{2}-14 v_{3} \alpha^{6} x^{3}+O\left(x^{4}\right) \\
k(y, \alpha) & =y+\left(1-4 v_{3} \alpha^{7}\right) y^{2}-14 v_{3} \alpha^{7} y^{3}+O\left(y^{4}\right) \\
k^{-1}(y, \alpha) & =y+\left(4 v_{3} \alpha^{7}-1\right) y^{2}+\left(2-2 v_{3} \alpha^{7}\right) y^{3}+O\left(y^{4}\right) \\
\left(\ell_{F}\right)^{\prime}\left(\alpha k^{-1}(y, \alpha)\right) & =1+O\left(y^{7}\right) \\
\left(k^{-1}\right)^{\prime}(y, \alpha) & =1+\left(8 v_{3} \alpha^{7}-2\right) y+\left(6-6 v_{3} \alpha^{7}\right) y^{2}+O\left(y^{3}\right) \\
f_{2}(\alpha) & =6-6 v_{3} \alpha^{7} \\
h_{2}(\alpha) & =3 \\
f\left(P\left(\mathbb{C P}^{2}\right)\right) & =375 v_{3} \alpha^{3} \\
& \equiv v_{3} \alpha^{3} .
\end{aligned}
$$

Here the last congruence follows because, in the ring $S[\alpha] /[2]_{F}(\alpha)$,

$$
2 \alpha v_{3} \equiv 127 v_{3}^{2} \alpha^{8} \equiv 0
$$

because $v_{3}^{2}=0$. Finally, $P\left(\mathbb{C P}^{2}\right)$, in $B P_{*} \llbracket\left[\alpha \rrbracket /[2]_{F}(\alpha)\right.$ mod decomposables, can only involve $v_{3}, v_{4}$, and higher, so we find $P\left(\mathbb{C P}^{2}\right) \equiv v_{3} \alpha^{7} \bmod$ decomposables and higherorder terms in $\alpha$ as desired. 


\section{References}

$\left[\mathrm{ABG}^{+} 14\right]$ Matthew Ando, Andrew J. Blumberg, David Gepner, Michael J. Hopkins, and Charles Rezk, An $\infty$-categorical approach to R-line bundles, $R$-module Thom spectra, and twisted R-homology, J. Topol. 7 (2014), no. 3, 869-893. MR 3252967

[Ada66] J. F. Adams, On the groups $J(X)$. IV, Topology 5 (1966), 21-71. MR 0198470 (33 \#6628)

[Ada74] Stable homotopy and generalised homology, University of Chicago Press, Chicago, Ill., 1974, Chicago Lectures in Mathematics.

[AL17] Vigleik Angeltveit and John A. Lind, Uniqueness of BP $\langle n\rangle$, J. Homotopy Relat. Struct. 12 (2017), no. 1, 17-30. MR 3613020

[And95] Matthew Ando, Isogenies of formal group laws and power operations in the cohomology theories $E_{n}$, Duke Math. J. 79 (1995), no. 2, 423-485. MR 1344767 (97a:55006)

[Ang08] Vigleik Angeltveit, Topological Hochschild homology and cohomology of $A_{\infty}$ ring spectra, Geom. Topol. 12 (2008), no. 2, 987-1032. MR 2403804 (2009e:55012)

[AR02] Christian Ausoni and John Rognes, Algebraic K-theory of topological Ktheory, Acta Math. 188 (2002), no. 1, 1-39.

[Baa73] Nils Andreas Baas, On bordism theory of manifolds with singularities, Math. Scand. 33 (1973), 279-302 (1974). MR 0346824

[Bak14] Andrew Baker, BP: close encounters of the $E_{\infty}$ kind, J. Homotopy Relat. Struct. 9 (2014), no. 2, 553-578. MR 3258694

[Bak15] - Power operations and coactions in highly commutative homology theories, Publ. Res. Inst. Math. Sci. 51 (2015), no. 2, 237-272. MR 3348109

[Bas99] M. Basterra, André-Quillen cohomology of commutative S-algebras, J. Pure Appl. Algebra 144 (1999), no. 2, 111-143. MR 1732625 (2001b:55025)

[BE74] M. G. Barratt and Peter J. Eccles, $\Gamma^{+}$-structures. I. A free group functor for stable homotopy theory, Topology 13 (1974), 25-45. MR 0348737

[Ber97] Clemens Berger, Combinatorial models for real configuration spaces and $E_{n}$-operads, Operads: Proceedings of Renaissance Conferences (Hartford, CT/Luminy, 1995), Contemp. Math., vol. 202, Amer. Math. Soc., Providence, RI, 1997, pp. 37-52. MR 1436916

[BJ02] Andrew Baker and Alain Jeanneret, Brave new Hopf algebroids and extensions of MU-algebras, Homology Homotopy Appl. 4 (2002), no. 1, 163-173. MR 1937961 (2003g:55012) 
[BM13] Maria Basterra and Michael A. Mandell, The multiplication on BP, J. Topol. 6 (2013), no. 2, 285-310. MR 3065177

[BMMS86] R. R. Bruner, J. P. May, J. E. McClure, and M. Steinberger, $H_{\infty}$ ring spectra and their applications, Lecture Notes in Mathematics, vol. 1176, SpringerVerlag, Berlin, 1986. MR 836132 (88e:55001)

[BP66] Edgar H. Brown, Jr. and Franklin P. Peterson, A spectrum whose $Z_{p}$ cohomology is the algebra of reduced $p^{\text {th }}$ powers, Topology 5 (1966), 149-154. MR 0192494

[Bru01] Robert R. Bruner, Extended powers of manifolds and the Adams spectral sequence, Homotopy methods in algebraic topology (Boulder, CO, 1999), Contemp. Math., vol. 271, Amer. Math. Soc., Providence, RI, 2001, pp. 4151. MR 1831346

[CJS95] R. L. Cohen, J. D. S. Jones, and G. B. Segal, Floer's infinite-dimensional Morse theory and homotopy theory, The Floer memorial volume, Progr. Math., vol. 133, Birkhäuser, Basel, 1995, pp. 297-325. MR 1362832 (96i:55012)

[CLM76] Frederick R. Cohen, Thomas J. Lada, and J. Peter May, The homology of iterated loop spaces, Lecture Notes in Mathematics, Vol. 533, SpringerVerlag, Berlin-New York, 1976. MR 0436146

[CM15] Steven Greg Chadwick and Michael A. Mandell, $E_{n}$ genera, Geom. Topol. 19 (2015), no. 6, 3193-3232. MR 3447102

[DK80a] W. G. Dwyer and D. M. Kan, Calculating simplicial localizations, J. Pure Appl. Algebra 18 (1980), no. 1, 17-35. MR 578563

[DK80b] , Function complexes in homotopical algebra, Topology 19 (1980), no. 4, 427-440. MR 584566

[EKMM97] A. D. Elmendorf, I. Kriz, M. A. Mandell, and J. P. May, Rings, modules, and algebras in stable homotopy theory, American Mathematical Society, Providence, RI, 1997, With an appendix by M. Cole. MR 97h:55006

[EM06] A. D. Elmendorf and M. A. Mandell, Rings, modules, and algebras in infinite loop space theory, Adv. Math. 205 (2006), no. 1, 163-228. MR 2254311 (2007g:19001)

[GH] Paul G. Goerss and Michael J. Hopkins, Moduli problems for structured ring spectra, available at: http://www.math.northwestern.edu//pgoerss/spectra/obstruct.pdf.

[GH04] Moduli spaces of commutative ring spectra, Structured ring spectra, London Math. Soc. Lecture Note Ser., vol. 315, Cambridge Univ. Press, Cambridge, 2004, pp. 151-200. MR 2125040 (2006b:55010) 
[GH15] David Gepner and Rune Haugseng, Enriched $\infty$-categories via nonsymmetric $\infty$-operads, Adv. Math. 279 (2015), 575-716. MR 3345192

[Goe99] Paul G. Goerss, Hopf rings, Dieudonné modules, and $E_{*} \Omega^{2} S^{3}$, Homotopy invariant algebraic structures (Baltimore, MD, 1998), Contemp. Math., vol. 239, Amer. Math. Soc., Providence, RI, 1999, pp. 115-174. MR 1718079

[Har02] John R. Harper, Secondary cohomology operations, Graduate Studies in Mathematics, vol. 49, American Mathematical Society, Providence, RI, 2002. MR 1913285

[HKM01] P. Hu, I. Kriz, and J. P. May, Cores of spaces, spectra, and $E_{\infty}$ ring spectra, Homology Homotopy Appl. 3 (2001), no. 2, 341-354, Equivariant stable homotopy theory and related areas (Stanford, CA, 2000). MR 1856030

[HSS00] Mark Hovey, Brooke Shipley, and Jeff Smith, Symmetric spectra, J. Amer. Math. Soc. 13 (2000), no. 1, 149-208. MR 1695653 (2000h:55016)

[JN10] Niles Johnson and Justin Noel, For complex orientations preserving power operations, p-typicality is atypical, Topology Appl. 157 (2010), no. 14, 2271-2288. MR 2670503

[Koc73] Stanley O. Kochman, Homology of the classical groups over the Dyer-Lashof algebra, Trans. Amer. Math. Soc. 185 (1973), 83-136. MR 0331386

[Kri95] I. Kriz, Towers of $E_{\infty}$-ring spectra with an application to BP, unpublished, 1995.

[Law17] T. Lawson, Calculating obstruction groups for E-infinity ring spectra, ArXiv e-prints (2017).

[Laz01] A. Lazarev, Homotopy theory of $A_{\infty}$ ring spectra and applications to $M \mathrm{U}-$ modules, $K$-Theory 24 (2001), no. 3, 243-281. MR 1876800 (2002k:55020)

[LMSM86] L. G. Lewis, Jr., J. P. May, M. Steinberger, and J. E. McClure, Equivariant stable homotopy theory, Lecture Notes in Mathematics, vol. 1213, Springer-Verlag, Berlin, 1986, With contributions by J. E. McClure.

[LN14] Tyler Lawson and Niko Naumann, Strictly commutative realizations of diagrams over the Steenrod algebra and topological modular forms at the prime 2, Int. Math. Res. Not. IMRN (2014), no. 10, 2773-2813. MR 3214285

[Lur09] Jacob Lurie, Higher topos theory, Annals of Mathematics Studies, vol. 170, Princeton University Press, Princeton, NJ, 2009. MR 2522659 (2010j:18001)

[Lur17] Higher Algebra, Draft version available at: http://www.math.harvard.edu/ lurie/papers/higheralgebra.pdf, 2017.

[Man12] Michael A. Mandell, The smash product for derived categories in stable homotopy theory, Adv. Math. 230 (2012), no. 4-6, 1531-1556. MR 2927347 
[May75] J. P. May, Problems in infinite loop space theory, Conference on homotopy theory (Evanston, Ill., 1974), Notas Mat. Simpos., vol. 1, Soc. Mat. Mexicana, México, 1975, pp. 111-125. MR 761724

[May77] J. Peter May, $E_{\infty}$ ring spaces and $E_{\infty}$ ring spectra, Lecture Notes in Mathematics, Vol. 577, Springer-Verlag, Berlin-New York, 1977, With contributions by Frank Quinn, Nigel Ray, and Jørgen Tornehave. MR 0494077 (58 \#13008)

[May01] J. P. May, Idempotents and Landweber exactness in brave new algebra, Homology Homotopy Appl. 3 (2001), no. 2, 355-359, Equivariant stable homotopy theory and related areas (Stanford, CA, 2000). MR 1856031

[Mil78] Haynes Miller, A spectral sequence for the homology of an infinite delooping, Pacific J. Math. 79 (1978), no. 1, 139-155. MR 526673

[MMSS01] M. A. Mandell, J. P. May, S. Schwede, and B. Shipley, Model categories of diagram spectra, Proc. London Math. Soc. (3) 82 (2001), no. 2, 441-512.

[MS] J. P. May and J. Sigurdsson, Parameterized homotopy theory, Preprint, http://www.math.uiuc.edu/K-theory/0716/.

[MS04] James E. McClure and Jeffrey H. Smith, Cosimplicial objects and little n-cubes. I, Amer. J. Math. 126 (2004), no. 5, 1109-1153. MR 2089084 (2005g:55011)

[MS16] Akhil Mathew and Vesna Stojanoska, The Picard group of topological modular forms via descent theory, Geom. Topol. 20 (2016), no. 6, 3133-3217. MR 3590352

[Pri70] Stewart B. Priddy, Koszul resolutions, Trans. Amer. Math. Soc. 152 (1970), 39-60. MR 0265437

[Pri75] Stewart Priddy, Dyer-Lashof operations for the classifying spaces of certain matrix groups, Quart. J. Math. Oxford Ser. (2) 26 (1975), no. 102, 179-193. MR 0375309

[Pri80] A cellular construction of BP and other irreducible spectra, Math. Z. 173 (1980), no. 1, 29-34. MR 584347

[PS59] F. P. Peterson and N. Stein, Secondary cohomology operations: two formulas, Amer. J. Math. 81 (1959), 281-305. MR 0124046

[Qui69] Daniel Quillen, On the formal group laws of unoriented and complex cobordism theory, Bull. Amer. Math. Soc. 75 (1969), 1293-1298.

[Qui71] Elementary proofs of some results of cobordism theory using Steenrod operations, Advances in Math. 7 (1971), 29-56 (1971). MR 0290382 (44 \#7566) 
[Rav86] Douglas C. Ravenel, Complex cobordism and stable homotopy groups of spheres, Pure and Applied Mathematics, vol. 121, Academic Press Inc., Orlando, FL, 1986. MR 860042 (87j:55003)

[Ric06] Birgit Richter, A lower bound for coherences on the Brown-Peterson spectrum, Algebr. Geom. Topol. 6 (2006), 287-308. MR 2199461

[Rob89] Alan Robinson, Obstruction theory and the strict associativity of Morava $K$-theories, Advances in homotopy theory (Cortona, 1988), London Math. Soc. Lecture Note Ser., vol. 139, Cambridge Univ. Press, Cambridge, 1989, pp. 143-152. MR 1055874

[Rob03] Gamma homology, Lie representations and $E_{\infty}$ multiplications, Invent. Math. 152 (2003), no. 2, 331-348. MR 1974890

[RW74] Douglas C. Ravenel and W. Stephen Wilson, The Hopf ring for complex cobordism, Bull. Amer. Math. Soc. 80 (1974), 1185-1189. MR 0356030

[Sen] Andrew Senger, On the realization of truncated Brown-Peterson spectra as $E_{\infty}$ ring spectra, unpublished.

[Sen17] A. Senger, The Brown-Peterson spectrum is not E_2 $\left(p^{2}+2\right)$ at odd primes, ArXiv e-prints (2017).

[Ste62] N. E. Steenrod, Cohomology operations, Lectures by N. E. STeenrod written and revised by D. B. A. Epstein. Annals of Mathematics Studies, No. 50, Princeton University Press, Princeton, N.J., 1962. MR 0145525

[Str99] N. P. Strickland, Products on MU-modules, Trans. Amer. Math. Soc. 351 (1999), no. 7, 2569-2606.

[tD68] Tammo tom Dieck, Steenrod-Operationen in Kobordismen-Theorien, Math. Z. 107 (1968), 380-401. MR 0244989

[Til16] Sean Tilson, Power operations in the Kunneth spectral sequence and commutative $\mathrm{HF}_{\mathrm{p}}$-algebras, 2016.

[Tur93] Paul R. Turner, Dyer-Lashof operations in the Hopf ring for complex cobordism, Math. Proc. Cambridge Philos. Soc. 114 (1993), no. 3, 453-460. MR 1235993

[Vog73] Rainer M. Vogt, Homotopy limits and colimits, Math. Z. 134 (1973), 11-52. MR 0331376 